\title{
ARCHITECTURAL MODELS AS A BASE FOR RELIABLE EARLY STAGE ENERGY SIMULATION
}

By

Jack Steele

A thesis submitted to the Victoria University of Wellington in fulfilment of the requirements for the degree of Master of Building Science

Victoria University of Wellington 



\section{Acknowledgements}

Firstly, to my supervisor, Dr. Michael Donn, thank you for the support, guidance, and feedback. For without, this thesis would not have been the same.

Thank you to Georgia Alexander, for coffee, feedback, and discussion into the depths of Excel.

Thank you to my family and friends, through providing me time to escape and play board games, I have remained sane.

Finally, a special thank you to my partner, Beccy Harding, your love, friendship, and support have helped me greatly in getting here. I am excited about our next adventure. 
Acknowledgements 


\section{Preface}

This thesis was submitted in fulfilment of the requirements of the degree for Master of Building Science to the School of Architecture, Victoria University of Wellington.

\section{Author}

Jack Steele

School of Architecture

Victoria University of Wellington

Email: j.steele987@gmail.com

\section{Supervisor}

Dr. Michael Donn

Associate Professor

School of Architecture

Victoria University of Wellington

Email: $\underline{\text { Michael.donn@vuw.ac.nz }}$ 


\section{Preface}




\section{Abstract}

\section{Abstract}

Designers from the Architecture, Engineering, and Construction (AEC) industries have shown a desire to allow for quantitative data to back up sustainable decision-making (Braasch, 2016). Methods and software used to reach this goal often do not provide all the information to make informed design decisions or require a complete remodelling of designs at each stage. These factors make Building Performance Simulation (BPS) feasible at early design stages, where it is most beneficial for Architects.

This thesis explores the current process to translate Architectural models constructed within a Building Information Modelling (BIM) environment into Building Energy Models (BEM) so that performance simulations can take place. Within the aim of exploring translation processes, the objectives were to document:

- Whether current processes can facilitate modelling of environmental building performance during early design, as well as during developed design?

- Whether there are any common problems or successful approaches that might form the basis of future improvements in the way Architect's and consultant's models work together?

This thesis has identified 19 translation processes from current literature and examined a range of representative processes for exchanging information between Architectural modelling and BPS programs. It concluded that translation issues can be classified into similar groups based on the overall processes used. The eight categories of issues can be used by future developers to determine their priorities in development, and those looking for a current solution can adopt one for themselves. None of the processes tested allowed for issue-free modelling of building performance during sketch design.

The two types of building translation schema evaluated in this thesis divided identified references between a dedicated and generalised approach. The dedicated approach of gbXML and the generic approach of IFC identified similar issues; however, IFC contained more of these problems because it communicates with all modelling programs at a lower information quality. Due to the generic approaches containing more issues that take longer to solve, it is currently more complicated to generate an energy model out of IFC data. While the gbXML schema can only provide benefits for BPS related translations, it is the most viable way to provide the service. 


\section{Contents}

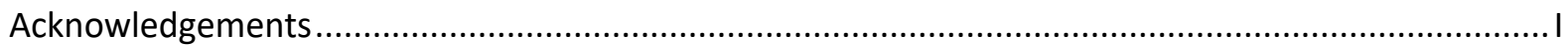

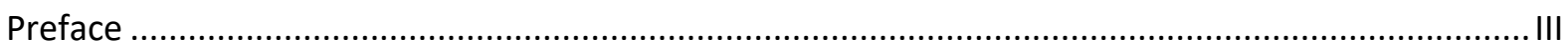

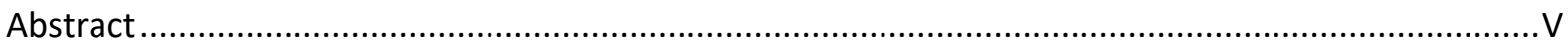

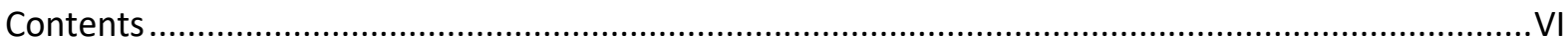

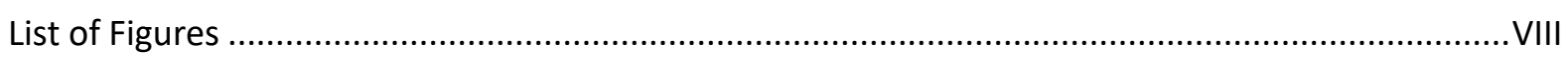

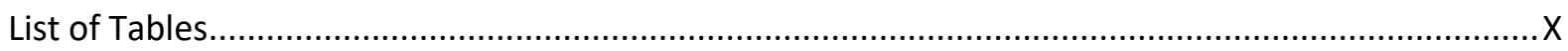

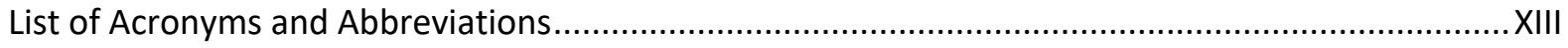

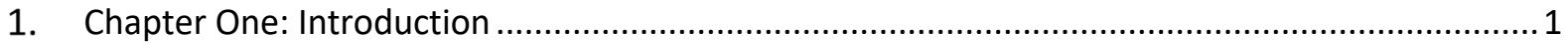

1.1 Methods of Building Information Translation ..............................................................

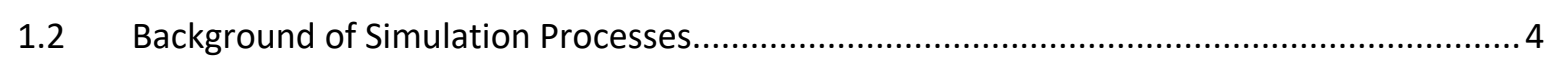

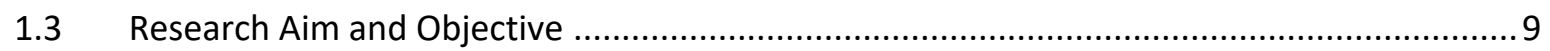

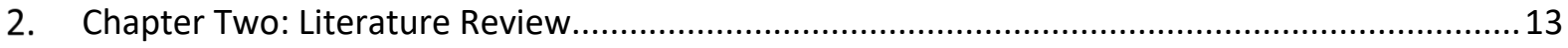

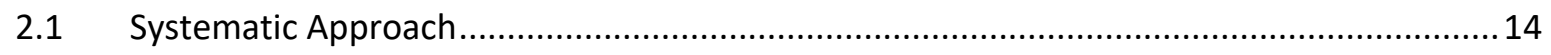

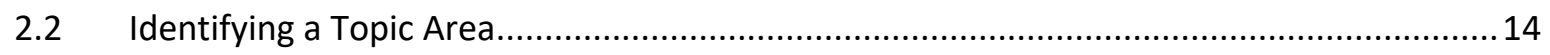

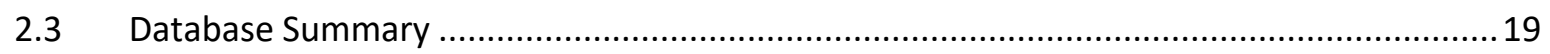

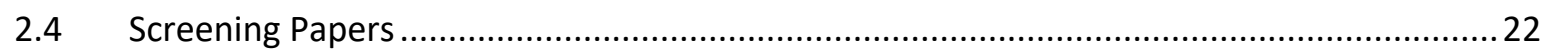

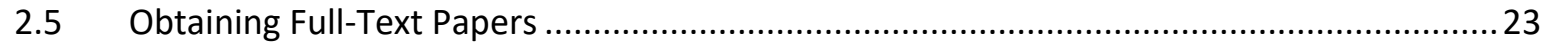

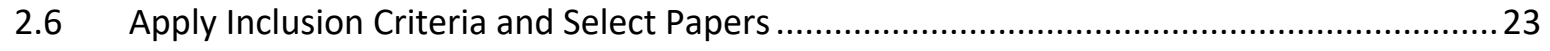

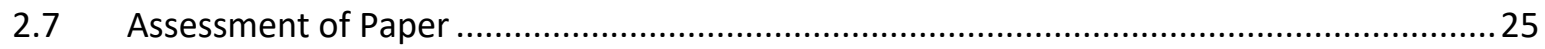

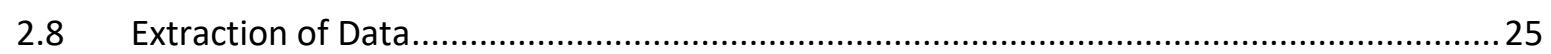

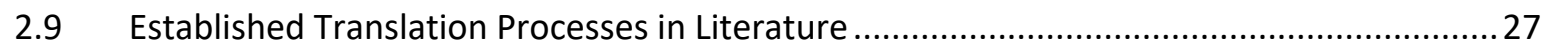

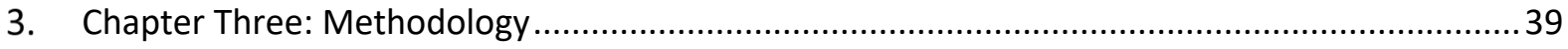

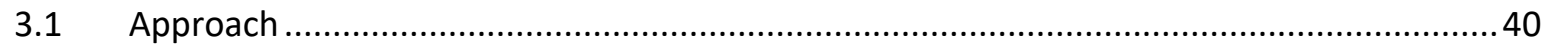

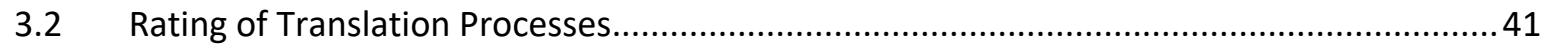

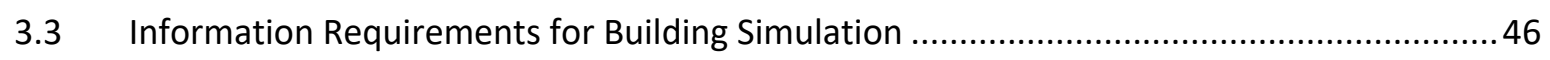

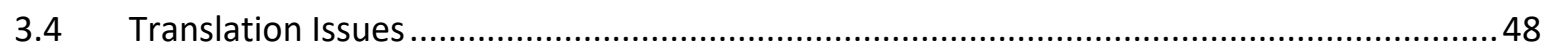

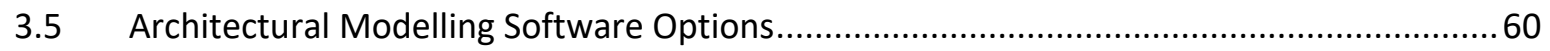

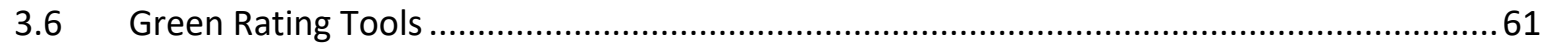

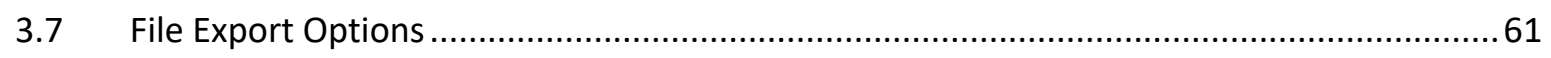

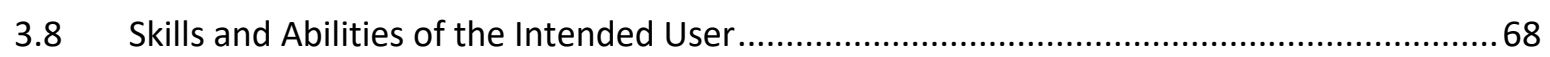

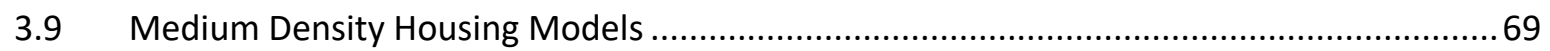

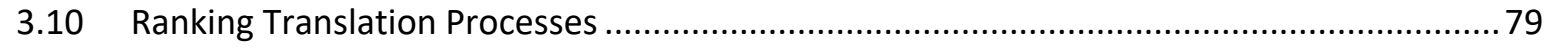

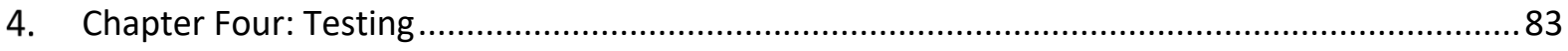

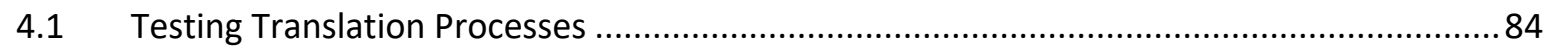




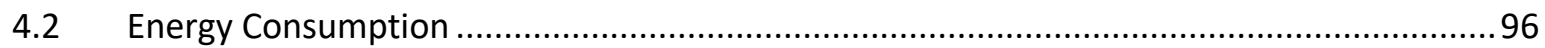

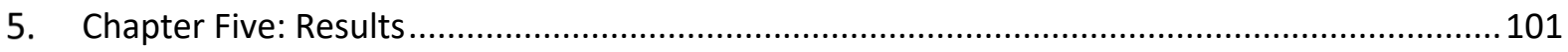

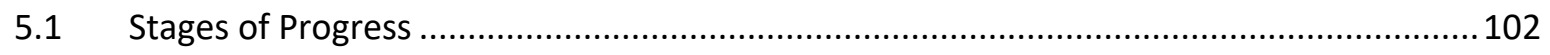

5.2 Number of Issues Identified from Testing Approaches .................................................... 103

5.3 Breakdown of Issues Identified from Each Approach ..................................................... 104

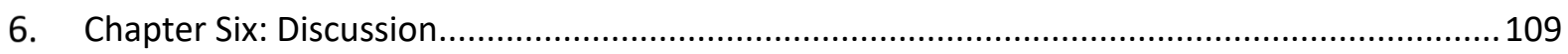

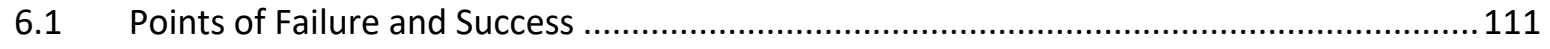

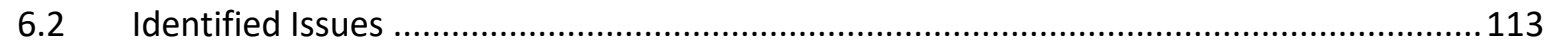

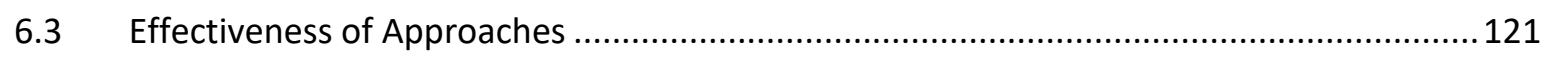

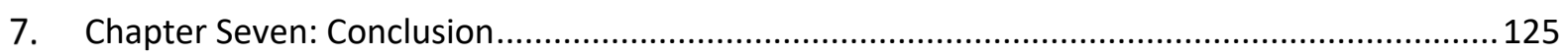

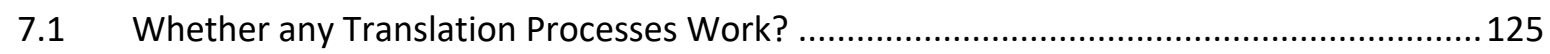

7.2 Whether there are any Common Problems or More Successful Approaches for Future

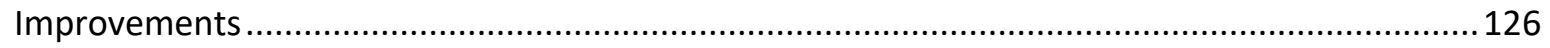

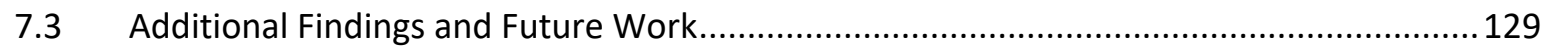

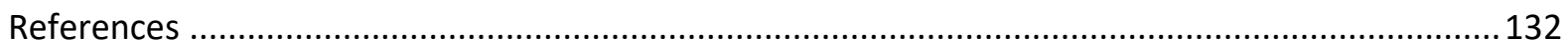

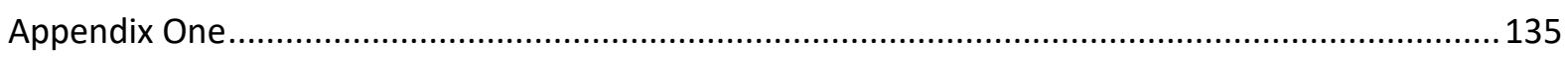

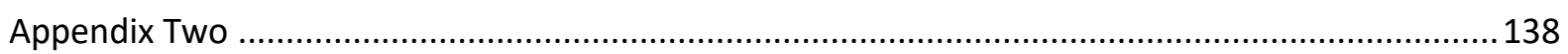

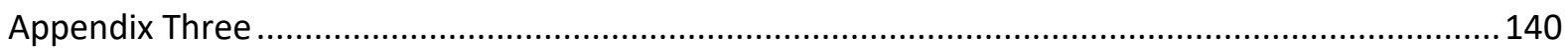




\section{List of Figures}

Figure 1: Simplified Simulation Approach from Braasch (2016) ......................................................

Figure 2: Model Methods as Described in Negendahl (2015) ..........................................................6

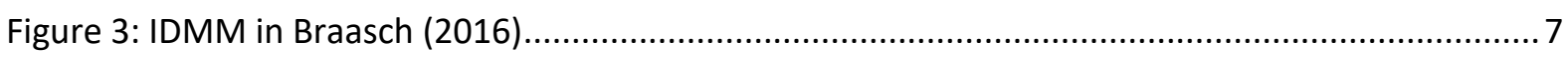

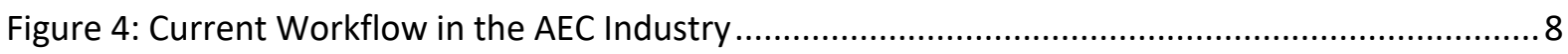

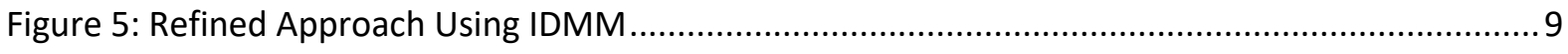

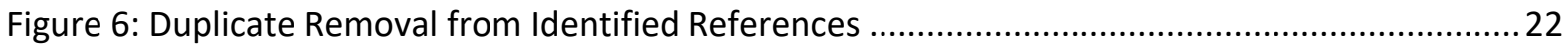

Figure 7: Applying Inclusion Criteria to Remaining References ....................................................23

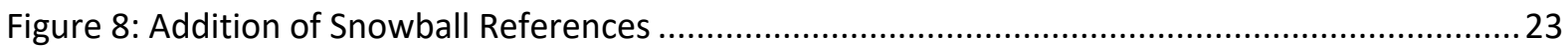

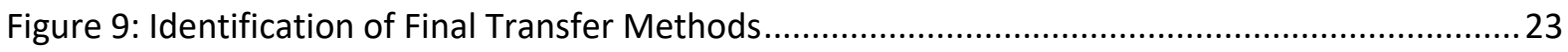

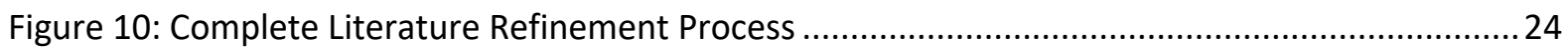

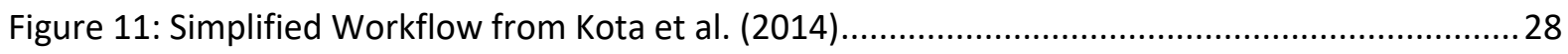

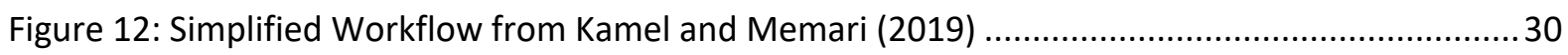

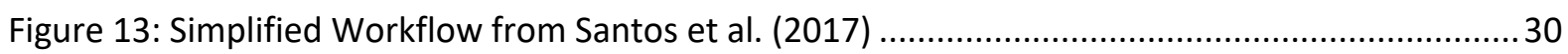

Figure 14: Simplified Workflow from Pratt et al. (2012) .......................................................................... 32

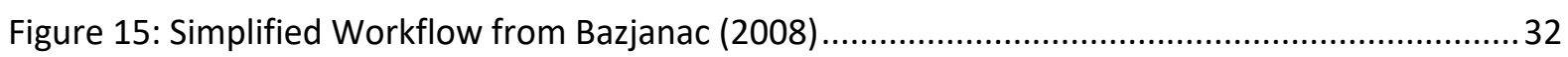

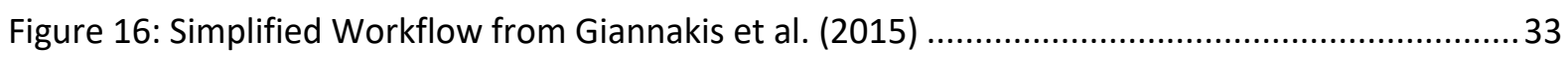

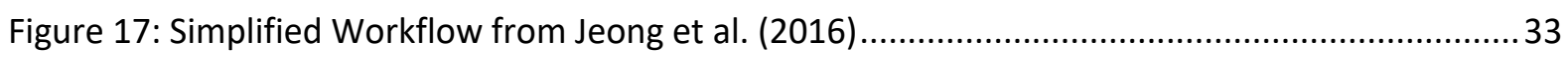

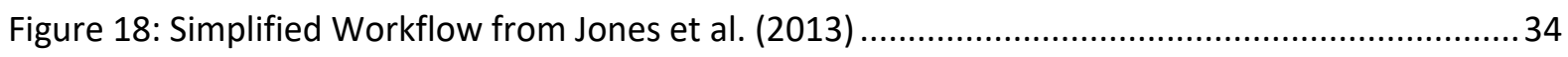

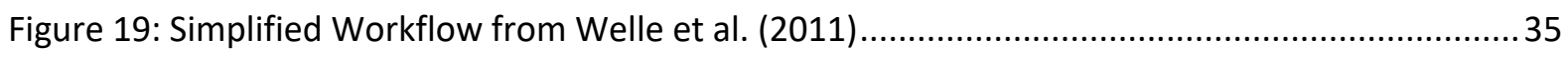

Figure 20: Simplified Workflow from Ramaji et al. (2016) ...............................................................36

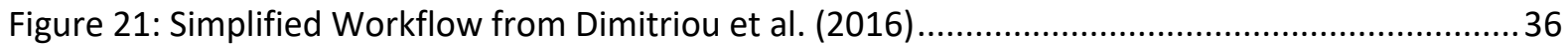

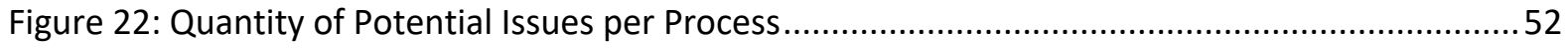

Figure 23: Specific Software or Program Used to Generate Potential Issues ....................................53

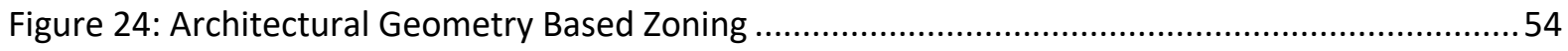

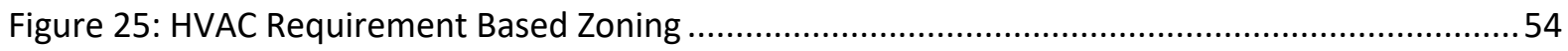

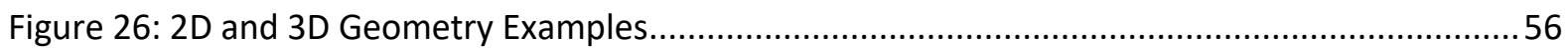

Figure 27: Surface Boundaries for Different Space Boundaries .....................................................57

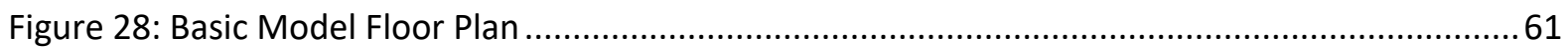

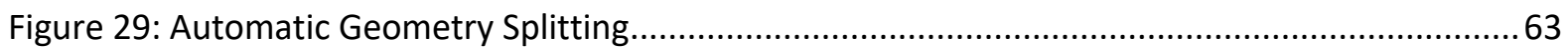

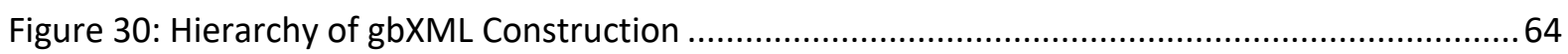

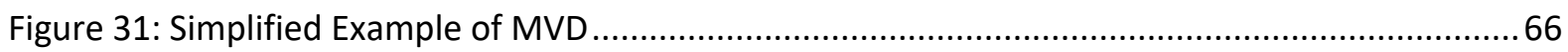


Figure 32: Hierarchy of IFC Construction

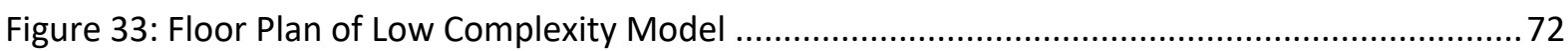

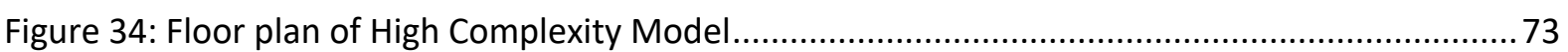

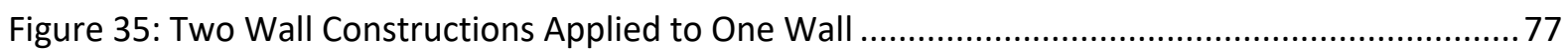

Figure 36: Layered Wall in Architectural Model (Left) and Condensed Wall (Right).........................78

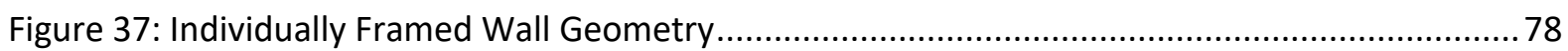

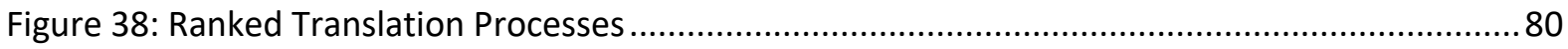

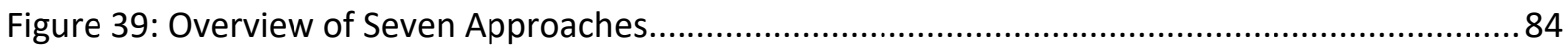

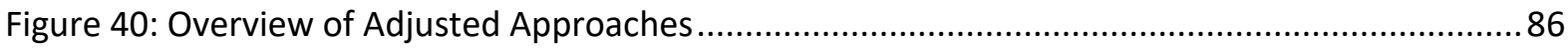

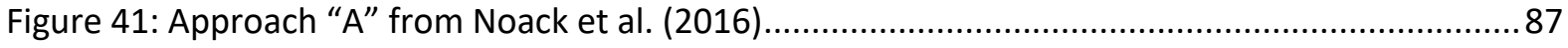

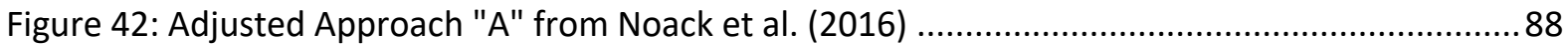

Figure 43: High Complexity Model Viewed in OpenStudio .....................................................8

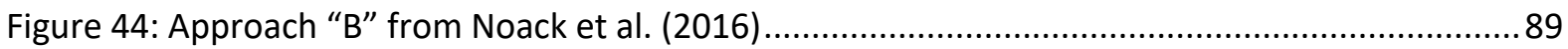

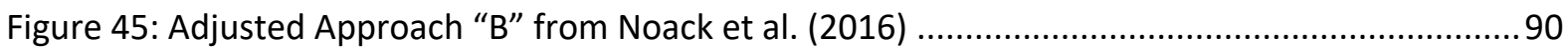

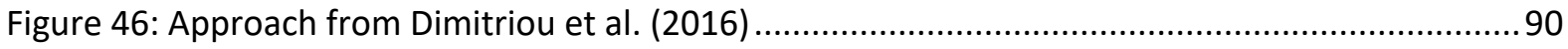

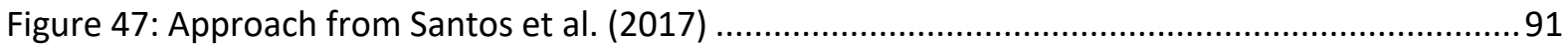

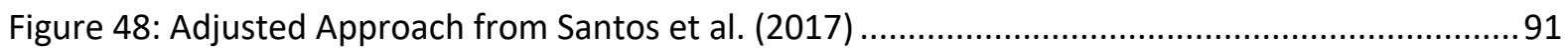

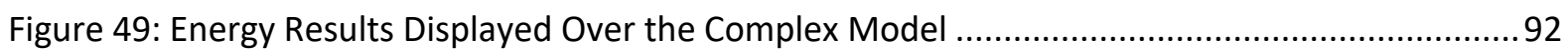

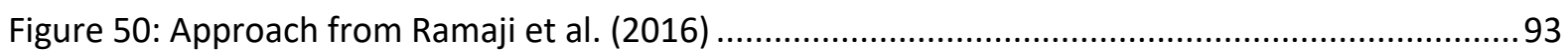

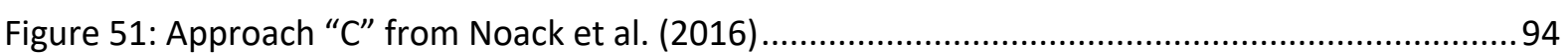

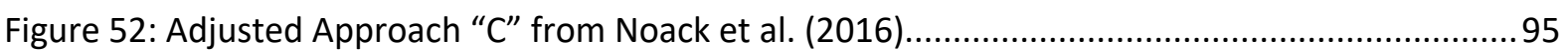

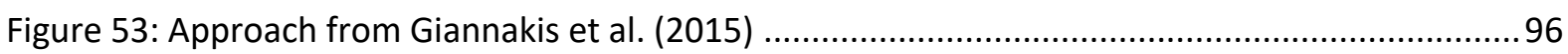

Figure 54: Energy Consumption Comparison of Low Complexity Model.......................................97

Figure 55: Energy Consumption Comparison of High Complexity Model .......................................99

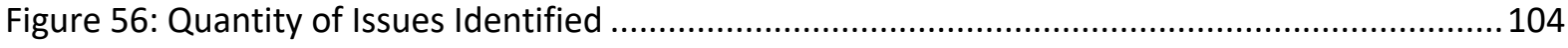

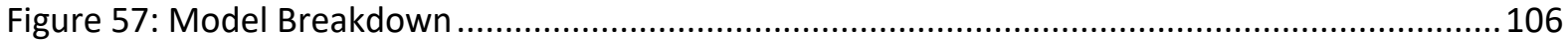

Figure 58: Ratio of Surfaces per Zone in Models .......................................................... 106

Figure 59: Low Complexity Model QA of Energy Consumption .................................................. 138

Figure 60: High Complexity Model QA of Energy Consumption ................................................. 138

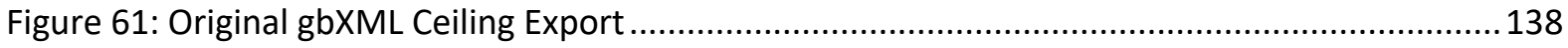

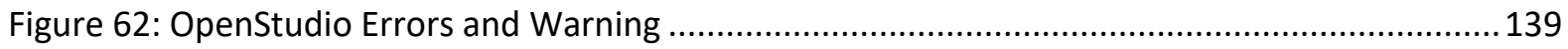

Figure 63: Incorrect Construction Details from IFC Import .................................................... 140 


\section{List of Tables}

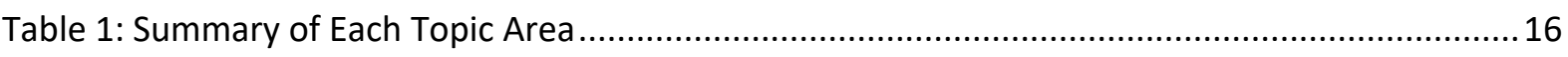

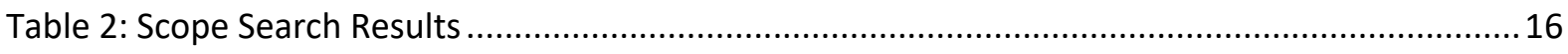

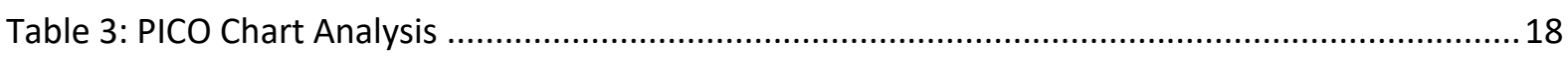

Table 4: Literature Review Database Summary ……...................................................................19

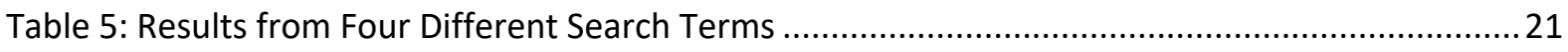

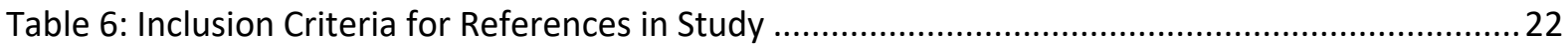

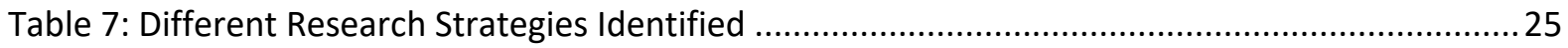

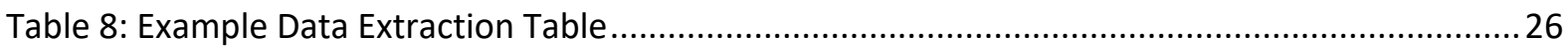

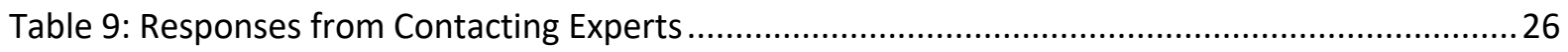

Table 10: Assessment of Translation Processes from Identified Literature ........................................27

Table 11: Ranking Requirements for the Measures of Success.......................................................4

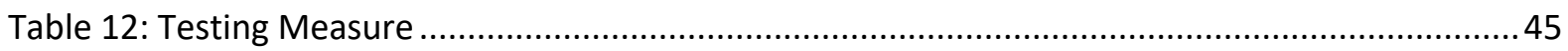

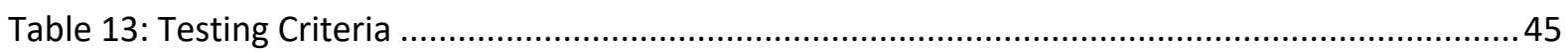

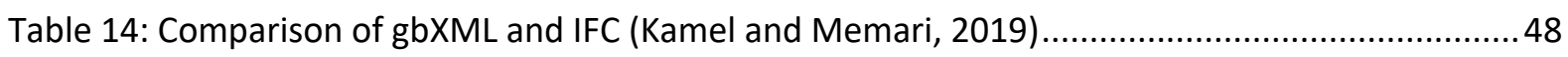

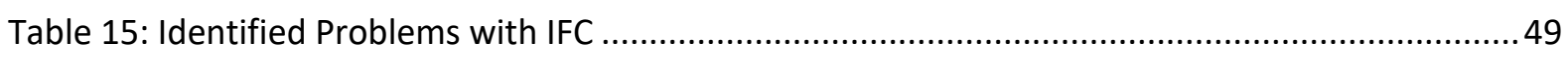

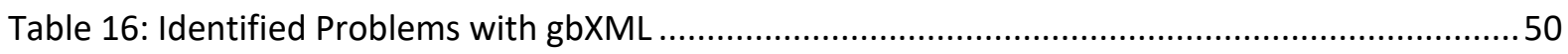

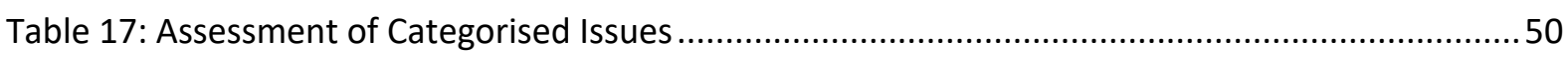

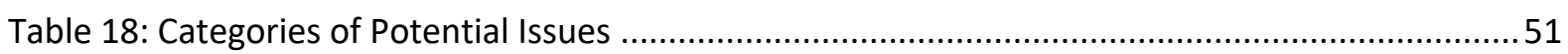

Table 19: Information Exported using the gbXML Space Export Option............................................62

Table 20: Information Exported using the gbXML Room Export Option ............................................63

Table 21: Information Exported using the gbXML Energy Export Option ............................................63

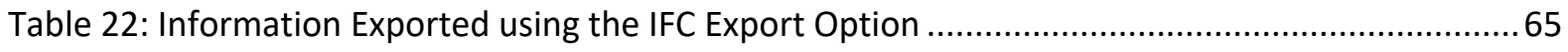

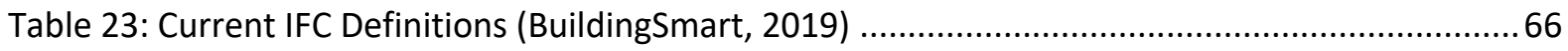

Table 24: IFC Extensions for Specific Tasks (BuildingSmart, 2019) ..................................................66

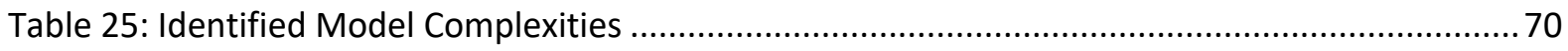

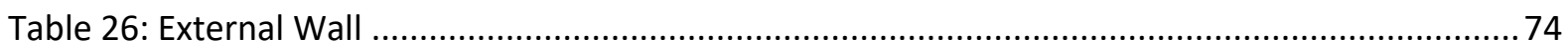

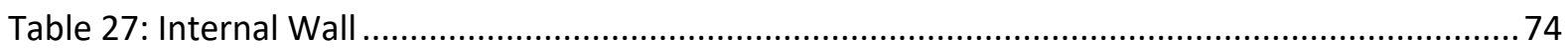

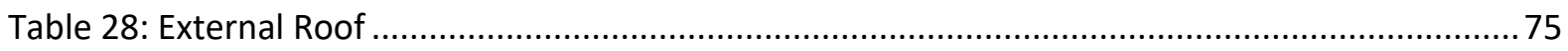

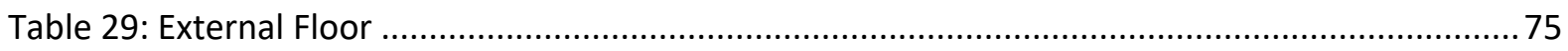

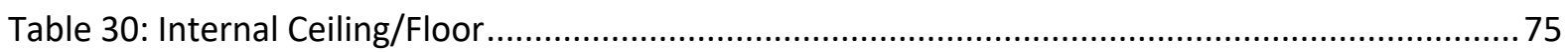

Table 31: Core R-values for Each Element ..................................................................................... 76 


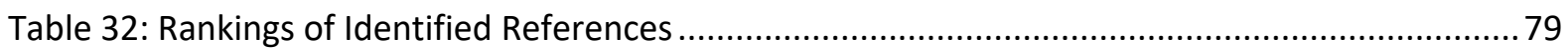

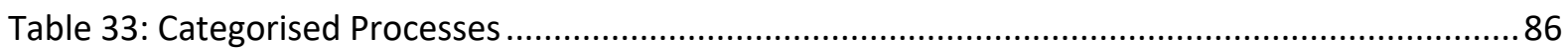

Table 34: Summary of Approach "A" from Noack et al. (2016) ….........................................................8 87

Table 35: Summary of Approach "B" from Noack et al. (2016) .............................................................. 89

Table 36: Summary of Approach from Dimitriou et al. (2016) .........................................................90

Table 37: Summary of Approach from Santos et al. (2017) .................................................................

Table 38: Summary of Approach from Ramaji et al. (2016) .............................................................93

Table 39: Summary of Approach "C" from Noack et al. (2016)............................................................94

Table 40: Summary of Approach from Giannakis et al. (2015) ........................................................96

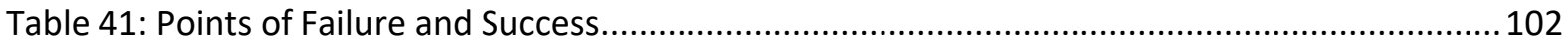

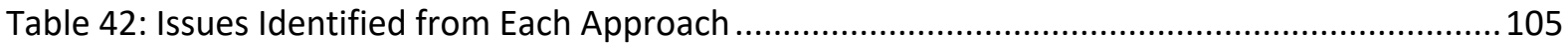

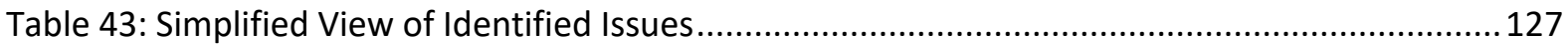

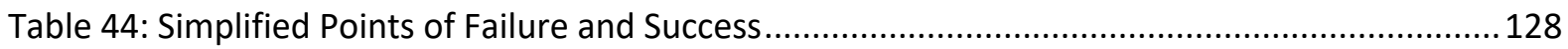

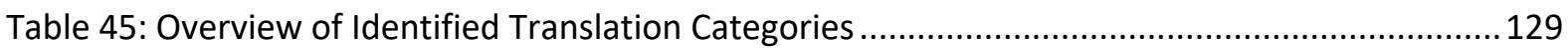

Table 46: Issues and Assessment from Identified References ........................................................... 135

Table 47: Criteria for Architecturally Friendly Simulation Tools (Weytjens and Verbeeck, 2010) ..... 136

Table 48: Insulation Requirements for Wellington, New Zealand (NZS4218, 2009) ......................... 137

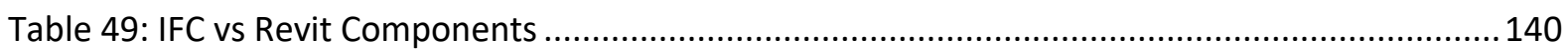

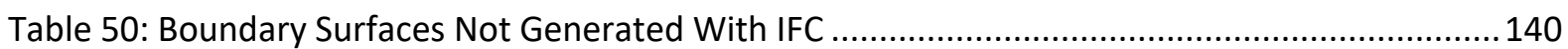

Table 51: Initial Energy Results from Ramaji et al. (2016)............................................................... 140 


\section{List of Tables}




\section{List of Acronyms and Abbreviations}

\section{General}

AEC

BEM

BIM

BPS

CAD

CBIP

CeMM

CoMM

DMM

GUI

HEEP

HVAC

IDMM

$\mathrm{MDH}$

PICO

VPL

1, 2, 3 LSB

\section{File Types}

EPW

gbXML

IDF

IFC

Software

EP

OSM
Architecture Engineering and Construction

Building Energy Model

Building Information Modelling

Building Performance Simulation

Computer Aided Design

Common Boundary Intersection Projection (CBIP)

Central Model Method

Combined Model Method

Distributed Model Method

Graphical User Interface

Household Energy End-use Project

Heating Ventilation and Air Conditioning

Integrated Dynamic Model Method

Medium Density Housing

Population, Intervention, Comparator, and Outcome

Visual Programming Language

First, Second, or Third Level Space Boundary

EnergyPlus Weather

Green Building eXtended Mark-up Language

Intermediate Data Format

Industry Foundation Class

EnergyPlus

OpenStudio Model 
List of Acronyms and Abbreviations 


\section{Chapter One: Introduction}

A scenario was imagined at the beginning of Braasch (2016). She asks us to think of an Architect who is approached to design a sustainable office building. The client wants high energy efficiency and outstanding internal lighting. How can the Architect ensure their design will meet these requirements? Braasch's answer to this involved moving early design in Architecture into a new space that allows Building Performance Simulation (BPS) to take away the uncertainty of performance.

Braasch (2016) identified a method of enabling BPS to work within a modelling environment that is familiar to Architects and Engineers. However, in current practice the processes would not work effectively, because constant updates to the Architectural model and the Building Energy Model (BEM) ensures they quickly become models of very different buildings and users get stuck in a iterative cycle of model-simulate-evaluate-remodel (Santos et al., 2017). Ensuring these models are up to date with each other means simulation does not lag behind the design only providing results for outdated models. BPS must facilitate the generation of solutions quickly and be able to sorten the evaluation of analysis periods (Struck et al., 2009). Despite the promises of Building Information Modelling (BIM), that one BIM model helps coordinate Architectural modelling and Engineering modelling, the reality is the models are difficult to coordinate. The New Zealand BIM Acceleration Committee (2019) states:

"BIM models make it easy to accurately re-calculate performance following changes, encouraging further exploration and optimisation of different design options. Information associated with a model has many uses: Simulating aspects of the proposed asset's behaviour, such as structural, thermal, acoustic, lighting, and fire performance" (author's emphasis).

Their ideal scenario is not currently a reality. Architectural modelling software is not integrated into a system that allows for these processes. Interoperability as described by "re-calculating performance following changes" is also found not to be feasible with current processes (Kamel and Memari, 2019).

Energy consumption can be directly calculated in some Architectural modelling software. This information is not used to help the user design through identifying problem areas, instead it is used for green rating tool compliance. Usually the compliance is for a tool in a specific region, with little adaptability for use elsewhere (e.g. Compliance with the North American LEED standard). 
Braasch demonstrated a desire amongst Architects and Engineers to use Distributed Energy and Daylight Modelling Tools in early design development. In this thesis the scenario Braasch (2016) imagined was explored again. The focus here was on examining the state of the art, in the integration of the Architect's model into a BEM approach. So that the complex and often manual processes needed to exchange information between the Architectural and energy models can be understood and perhaps simplified, or even automated.

There are three basic types of building information transfers:

1) A combined approach where simulations are conducted within Architectural software

2) A dedicated approach that focuses on one specific area of information translation

3) A generic BIM approach that translates all available information

Only the dedicated and generic approaches are evaluated in this thesis. The combined approach limits the potential though specifying what simulations can be performed and focusing results towards specific industry standards that are not applicable worldwide.

As there is no dominant process for integrating Architect's models into BEM, there are likely many issues causing inefficient processes. Exposing the current issues and potential solutions can allow developers to focus on specific issues or provide current users with a workaround solution. 


\subsection{Methods of Building Information Translation}

\subsubsection{Building Information Modelling}

Building Information Modelling (BIM) enables separate disciplines from across the Architecture, Engineering, and Construction (AEC) industries to use one-model for storage of facility documentation, retrieval of analytical information, and knowledge sharing (NBIMS, 2015). Recent research on the implementation of BIM models within this industry shows that a one-model approach provides major benefits over multiple fragmented building models (Ganda, 2019). The benefits stem from improved coordination, along with everyone accessing and developing the same model. Additional benefits come with Designers and Practitioners not having to create separate models for specific analysis and then needing to consistently update and re-analyse due to changes in the original model. Moreover, if inconsistencies between models go unnoticed until the construction stage, the delay to time and increase to cost is extensively higher. Popular software such as Revit and ArchiCAD promote and market themselves as BIM models, while they are just Computer Aided Design (CAD) modelling tools that can collate information into a BIM file making them a BIM authoring tool.

The difficulty with having everything stored in one data source is retrieving specific sets of information. Ganda (2019) identifies that for energy modelling, exporting a BIM model from an Architectural model does not lead to a functional energy model. A separate energy model must be created outside of the BIM process. If solved, Practitioners would be able to edit the BIM model and automatically receive updated energy model results.

The big issue with a BIM model is coordination. The priority should be ensuring the BEM is representative of the current design model, not of the design two iterations ago when the energy model was created independently of the Architectural model. To identify the different issues with current processes a review of current literature around known problems relating to information translation was completed. Then identifying current transfer processes for converting Architectural model into BEM from the literature. While the last step is to test these identified processes and categorise the issues so that solutions are given. An experienced energy modeller may prefer to create a model where they can directly control all the inputs and variables. An approach that encourages using BIM can provide those valid inputs and variables, making the process easier without time-consuming model replication. 


\subsubsection{Dedicated Approach Using gbXML}

Green Building eXtended Mark-up Language (gbXML) files are a type of dedicated translator that only focuses on translating an Architect's model to a BEM. It requires that an individual exporter needs to be written for each Architectural modelling program, in addition to, a reader for each analytical program.

\subsubsection{Generic Approach Using IFC}

Industry Foundation Class (IFC) files are a part of the generic BIM approach that aims to translate as much information as possible from an Architect's model. A generic export that contains everything a model has to offer, though at a lower level of quality, it also only requires analytical programs to be able to read the file.

\subsection{Background of Simulation Processes}

Braasch (2016) determines that the Distributed Model Method (DMM) has the highest potential benefit from the model methods that Negendahl (2015) identifies. DMM is one of four model methods that illustrate how different software can interact and exchange information. In this section, Braasch's approach was analysed to express where its shortfalls are, so improvements can be made. As well as identifying the benefits and limitations of Negendahl's model methods.

Focus groups with Architects and Engineers were conducted by Braasch (2016) to understand whether a single software set could allow environmental BPS to be run simpler and meet specific requirements. In this study, modelling software (Rhino) and a Visual Programming Language (VPL) software (Grasshopper) were used to integrate modelling and analysis. A VPL is a programming method that uses visual elements or nodes, which can be linked to each other in a sequence. The process demonstrated in the focus groups is presented in Figure 1.

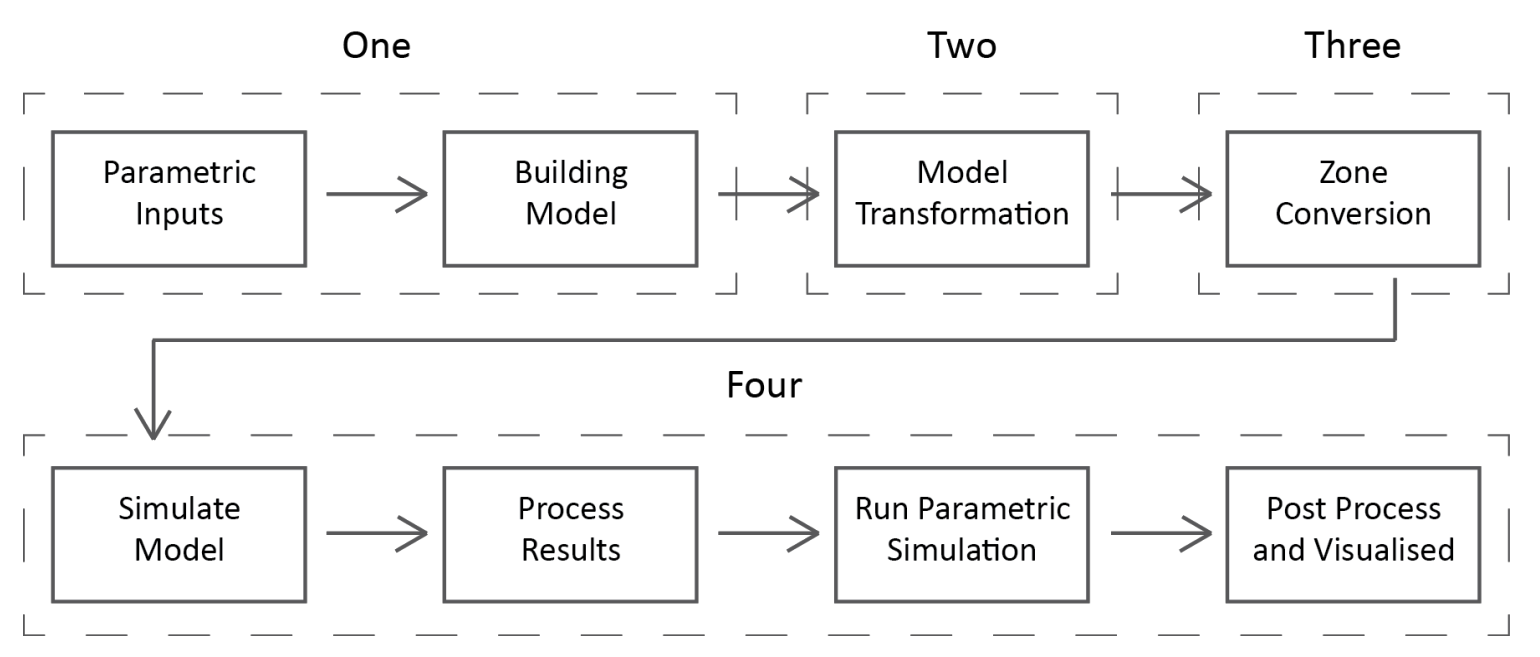

Figure 1: Simplified Simulation Approach from Braasch (2016) 
The eight processes from Braasch (2016) can be simplified to four essential steps required for any model simulation based off an Architectural model:

1. Construct Building Geometry within Architectural modelling Software - Each building or floor needs to be modelled as a planar volume

2. Transfer Model - A model needs to be translated into a BEM that can be read by simulation software

3. Zone Conversion - Boundary representations must be converted into zoned spaces based on what is set in the Architectural model

4. Simulation \& Results - This process varies, depending on what environmental conditions are being analysed, while results of this can be analysed based on numerical readouts

Braasch (2016) proposed an example of DMM, this along with the Central Model Method (CeMM), Combined Model Method (CoMM), and the Integrated Dynamic Model Method (IDMM) are processes to allow an Architectural model to be coupled together with BPS tools (Negendahl, 2015). 

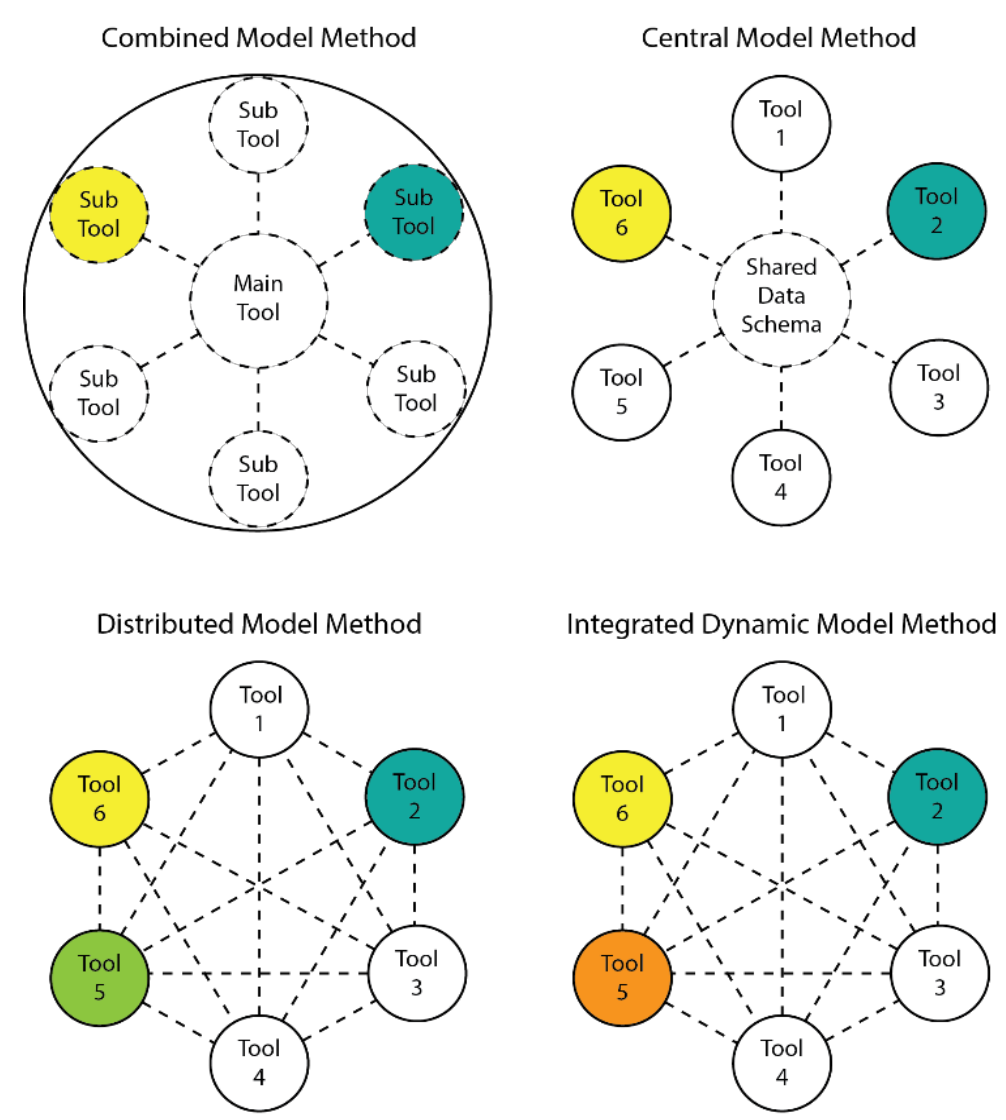

Integrated Dynamic Model Method

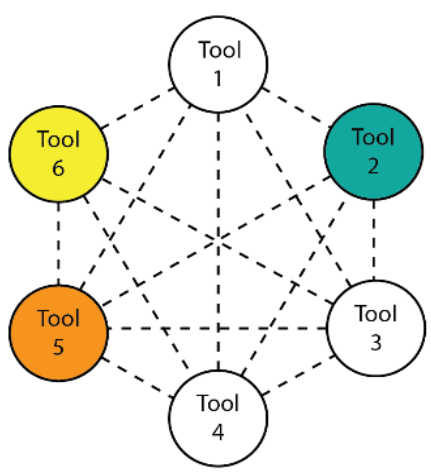

Design Tool (CAD)

Middleware

Calculation Tool (BPS)

Middleware (VPL)

Figure 2: Model Methods as Described in Negendahl (2015)

The CoMM methodology was used due to its operation as an all in one simulation package. Programs such as Revit and ArchiCAD promote themselves as a CeMM, whereas they contain sub tools that are not fully-fledged simulation programs, making them a CoMM. By containing multiple sub tools under one larger main tool, the software can handle modelling and simulation at the same time. The disadvantages to having such high interoperability are that users are restricted to specific options or features that are offered by the software. As the combined model is essentially all or nothing, it cannot support multiple design tools e.g. energy simulation and lighting, but may be faster and more reliable due to this as well (Negendahl, 2015).

The CeMM focuses on a unique data schema or central database for information to be stored and shared. Having distinct Architectural modelling and BEM tools allows the software to be independently developed to the highest-level while being able to read and write to the same model. This process of linking around a central framework is most often referred to as BIM, and commonly uses files based on the Industry Foundation Class (IFC) or Green Building eXtended Mark-up Language (gbXML) schemas. A disadvantage of this methodology was that "collaboration 
within centralized models has been considered time-consuming and, in some cases, counterproductive in terms of design exploration". Model simulation speed here depends on the client and what systems have been integrated, there was either bias toward feedback, reaction time, or accuracy (Negendahl, 2015).

DMM and IDMM are very similar, and are only distinguished by the type of Middleware used. When the Middleware program has a VPL component that allows additional information to be added by a user, it is considered an IDMM, while any other Middleware that adjusts or passes information is just a DMM. A range of various tools can be linked together with these methodologies, allowing bidirectional links with more than one design tool. If a VPL is used it can be operated by Architects, Engineers, simulation experts, or automatically by anyone. The downside to using a VPL is if not run automatically, Architects may struggle to run it, as even lowlevel programming skills are relatively uncommon (Weytjens and Verbeeck, 2010). While there are higher risks of un-validated software, the usability and level of customisation are also much higher. Allowing commercial plugins to be used encourages users to customise and optimise the model environment to fit specific needs and requirements. Automation is also a feature that is solely restricted to methods within DMM or IDMM due to VPLs being able to be used (Negendahl, 2015).

The simulation process discussed by Braasch is a DMM, but more specifically an IDMM based off Negendahl's definition. Figure 3 shows how Rhino transfers geometric information through Grasshopper, which is a VPL that has plugins; this allows calling of all sorts of simulation.

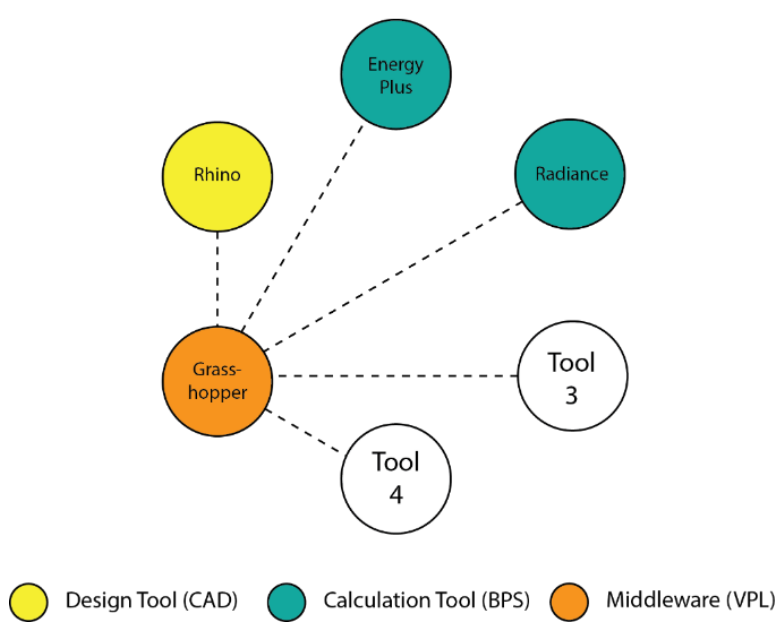

Figure 3: IDMM in Braasch (2016)

This process enables many different simulations to be conducted on one model's geometry. By only looking at what Ladybug Tools can provide through its plugins, simulations can be used to provide information around; annual daylighting, electric lighting, energy consumption, natural 
ventilation, renewables, urban heat island, indoor comfort, outdoor comfort, and the potential for many more (Roudsari and Mackey, 2019).

What Negendahl (2015) defines as IDMM is in fact not that integrated in terms of the industry, its only integration is between BPS software and geometry. For a method to be fully integrated, Architectural design phases must be a part of the process.

Architects are not using Rhino as a design tool so a simulation specialist must develop a separate model. This work is usually outsourced, which adds to the cost and time of a project. During earlystage concept development when a design is most malleable, concepts can be changing daily. Therefore, when an outsourced consultant returns with simulation results, they are already redundant. With early intervention, simulation results can be used to enhance the Architect's confidence in designs instead of just using them to reach green building criteria (Xie and Gou, 2017). To solve this problem, the implementation of a faster and more efficient process route is required. Figure 4 shows the current industry approach.

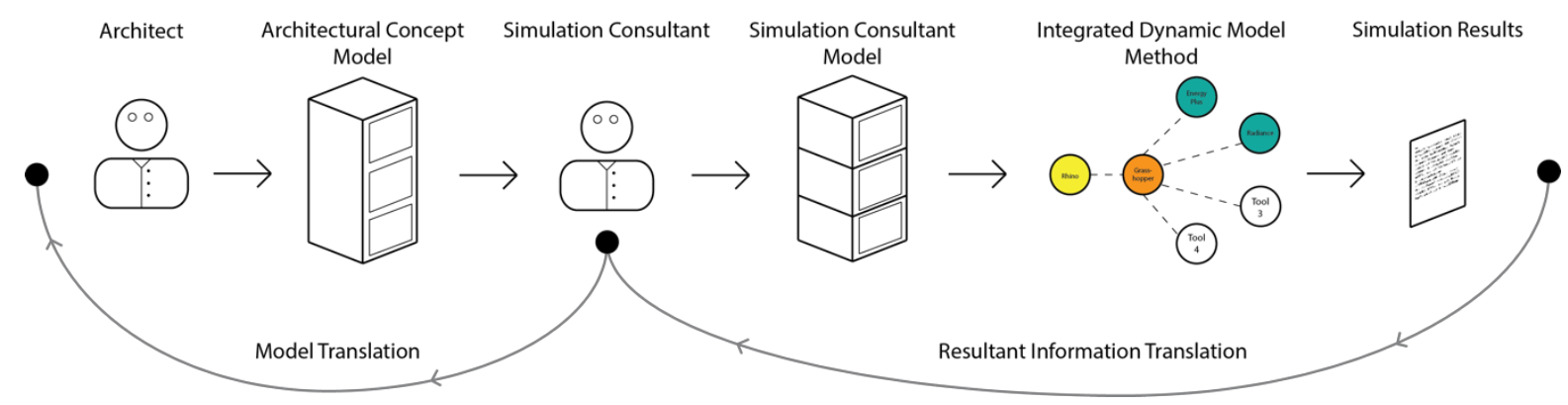

Figure 4: Current Workflow in the AEC Industry

Due to the current process, not only is the Architectural concept model not useful for a simulation consultant, but it is also not useful for an Engineer. At later stages of design when Engineers are historically getting involved, the produced Architectural model would not be trusted, as the inputs were not checked before modelling began. Therefore, an additional Engineering model is also created. Only $10 \%$ of consultants say they communicate results of calculations in a way that Architects can comprehend (Alsaadani and Bleil De Souza, 2018).

Allowing an Architectural model to be simulated automatically using the IDDM discussed in Braasch (2016) engages an Engineer at the start of a project. Then the simulation consultant steps can be removed making the process simplified, reliable, and likely to produce trustable models. The process workflow eliminates the extra time and associated cost with consultants being involved at an early design stage; in addition to ensuring designs work for the users. Currently, complex Architectural models struggle to retain all the information they contain when being transferred into a BEM. Through enabling Engineers to provide the Architect with a template, they 
can ensure an accurate BEM resulting from the Architectural modelling process. It can be used to inform the Architects own designs using numerical values and design suggestions. This step is based around the CeMM described earlier, where a shared data schema is used to transfer model information.

Engaging Engineers in the pre-design phases ultimately improves sustainable aspects of a building and allows a trusted model to be produced at the end. If Engineers and Architects work in parallel to produce a set of trusted materials, constructions, feature locations, building orientations, window sizes, shading sizes, and more for a base model. When it comes to conceptual Architectural models, simulating, and getting results the outcomes could be inherently trusted. This process is proposed in Figure 5. Alsaadani and Bleil De Souza (2018) show that $62 \%$ of Architects agree that BP is most beneficial to the design process if they conduct it during early design stages, and consultants follow it up. This enables what Braasch (2016) found, which is that Architects want to only model the design once and are innately not interested in ensuring accuracy. Engineers also sought easy to use software, but they want to be able to provide accurate models, which Architects could trust and modify to inform their design.

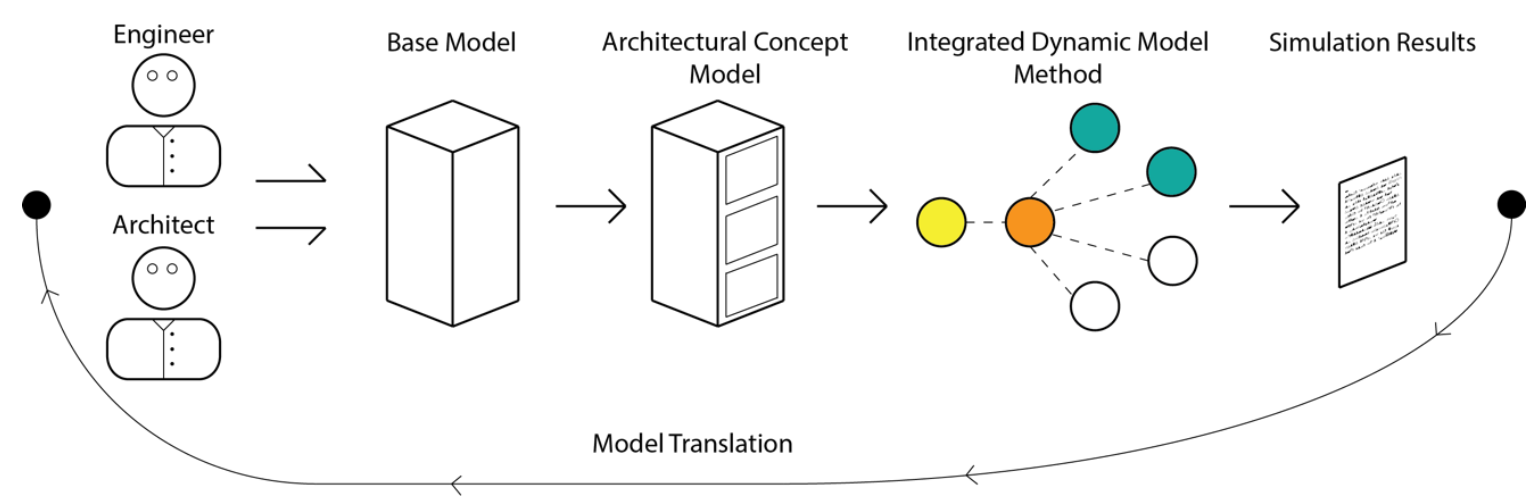

Figure 5: Refined Approach Using IDMM

\subsection{Research Aim and Objective}

As revealed in the next chapter, there have been many attempts to translate Architectural models into Building Energy Models. The aim of this thesis is to examine these in order to document:

- Whether current processes can facilitate modelling of environmental building performance during early design, as well as during developed design?

- Whether there are any common problems or successful approaches that might form the basis of future improvements in the way Architect's and consultant's models work together? 
The process for answering these questions begins by systematically examining the literature for translation procedures that have been developed and tested. The hypothesis is that for BPS based on an Architectural model to become mainstream in practice, translation must be quick, simple, and intuitive. By identifying where and how errors occur in a process, avoiding them can be used to design with good performance in mind. 
Chapter One: Introduction 
Chapter One: Introduction 


\section{Chapter Two: Literature Review}

The integration of Architects and Engineers into the BPS workflow described in the previous chapter has the potential to be successful based on the background of simulation processes. There have been two questions proposed, which are both prerequisites to answering the full research question developed in this chapter.

In this chapter, the process to obtain relevant references that contain translation processes is established. The literate review is completed in a systematic way, using the process by Boland et al. (2014) that has all searches, scopes, and criteria defined. The aim of this review is to understand how BIM is used in BPS currently and whether it is suited for an Architectural audience. 


\subsection{Systematic Approach}

A systematic literature review is a process that allows for the best possible evidence and sources to be identified around a research topic (Boland et al., 2014). The systematic approach used in this research thesis is established by Boland et al. (2014). An advantage for using a systematic review process is an understanding of different research methodologies and insight into the strengths and weaknesses of identified literature. The most important aspect of the whole literature review process is that it is planned, described, and transparent. This allows for the actions and decisions to be explicitly set out and scrutinised.

The process of a systematic literature review follows eight steps, listed below:

1. Performing scoping searches, identifying the review question, and writing a protocol: Scope searching is used to identify background literature, which helps in defining the research question, inclusion criteria, and exclusion criteria.

2. Searching for literature: Papers relating to the research question can be identified from reference databases or other unpublished sources.

3. Title and abstract screening: Screening the identified papers through reading the title and abstract and then determining whether they are relevant to the research question or not.

4. Obtaining identified papers: Full-text versions of each reference are obtained.

5. Determination of papers: Inclusion criteria are used to exclude any papers that do not meet the strict requirements.

6. Assessment of paper quality: Assess each paper for quality using a quality assessment tool.

7. Extraction of data: Extract important information that has been identified from each paper and summarise.

8. Analysis and amalgamation: Scrutinise the data found narratively or through metaanalysis.

\subsection{Identifying a Topic Area}

The topic area that this research thesis covers began development in 2018 during a university course focused on research methodologies. In this course two proposals were prepared, each with a differing focus. Proposal one was titled "Building performance simulation for Architects and Engineers" while proposal two which initiated from a different idea was titled "The effects and solutions for glazing relating to performance, comfort, and occupants". Proposal one was identified as the preferred research area. The proposal focused on Architects and Engineers using 
a Graphical User Interface (GUI) to conduct within their design process and reporting what they think and feel when using it. The scope for this proposal was too large and therefore it needed to develop further.

Over the summer break, the scope was refined to aim solely on Medium Density Housing (MDH) due to the target of 10,000 new MDH being constructed in New Zealand over the next ten years, through the Kiwi Build programme. In addition to, research priorities published by Building Research Association of New Zealand (BRANZ) showing that a need for research around giving industry the tools to deliver medium density housing that meets the needs of New Zealanders.

Further development of the research topic, lead to focusing on creating a tool which helped Designers improve energy consumptions, lighting levels, and the air quality of $\mathrm{MDH}$ through building optimisation. The proposed tool would take MDH and find the best site placements so that better internal environments could arise. Testing this process would have been through showing focus groups two different tools, one focused on BPS and the other on the GUI. However, reflection on this idea shows it was just tool development and not research.

More research into the topic and another scoping search revealed the different model methods for BPS. This led to the research question "Feasibility of early design stage building performance simulation tools to recommend design changes for Architects and Engineers: A comparison of the central model method and the integrated dynamic model method". This aimed to determine what positive and negative attributes are identified for each model method. Also, if there is another model method that is a combination of both concepts. This question incorporated ideas that needed to be researched, but it was also vague in terms of what type of BPS, as well as why it was focusing on Architects and Engineers. A key part of this research would have been researching and developing processes for energy simulation directly within Architectural modelling programs. After consulting the developers of Ladybug and Honeybee to understand where the industry is heading from their view, they responded saying the focus of many people was shifting from "how can we work within Autodesk's Dynamo ecosystem?" to "how can we get energy model geometry out of Revit as quickly and cleanly as possible?".

The current processes to translate Architectural model information to BEM cannot be used as a simple and reliable means of data transfer due to the lack of interoperability and complexity. Therefore, the generation and selection of high performing design alternatives during the early design stages must be produced through a methodology such as the IDMM. This led to the proposal of "Validation of the Integrated Dynamic Model Method to produce performance-based design alternatives using building performance simulation for early-stage design". This aimed to 
validate IDMM, determine what information was available during the early design stage, determine what information was needed to complete BPS, and determine what level of modelling resolution was needed for early-stage design. Validating a process was noted to be out of the scope of an Architectural science thesis.

The process that has been completed to refine and identify a topic area is summarised in Table 1. Each step new ideas are added and previous ones are refined.

Table 1: Summary of Each Topic Area

\begin{tabular}{|c|c|c|c|c|c|}
\hline Topic & 1 & 2 & 3 & 4 & 5 \\
\hline $\begin{array}{l}\text { Areas of } \\
\text { Interest }\end{array}$ & $\begin{array}{c}\text { GUI } \\
\text { BPS } \\
\text { Architects } \\
\text { Engineers }\end{array}$ & $\begin{array}{c}\text { GUI } \\
\text { BPS } \\
\text { Architects } \\
\text { Engineers } \\
+\mathrm{MDH}\end{array}$ & $\begin{array}{c}\text { GUI } \\
\text { BPS } \\
\text { MDH } \\
\text { + Optimisation } \\
\text { + Energy } \\
\text { + Lighting } \\
\text { + Designers }\end{array}$ & $\begin{array}{c}\text { BPS } \\
+ \text { Early Stage } \\
+ \text { Architects } \\
+ \text { Engineers } \\
+ \text { CoMM } \\
+ \text { IDMM }\end{array}$ & $\begin{array}{c}\text { BPS } \\
\text { Early Stage } \\
\text { Architects } \\
\text { IDMM } \\
+ \text { CAD to BEM } \\
+ \text { BIM to BEM }\end{array}$ \\
\hline Pro & Audience & Typology & $\begin{array}{c}\text { Specifies what is } \\
\text { tested }\end{array}$ & Stage of design & $\begin{array}{l}\text { Transfer } \\
\text { methods }\end{array}$ \\
\hline Con & Wide Scope & Wide Scope & Not research & $\begin{array}{c}\text { What is the } \\
\text { outcome }\end{array}$ & $\begin{array}{l}\text { Focusing on } \\
\text { IDMM }\end{array}$ \\
\hline
\end{tabular}

\subsubsection{Early Scope Search}

Areas of interest from the final identified topic are used in an early scope search to understand how much information there was on the topic. Four information databases are used: Scopus, Science Direct, ProQuest, and Google Scholar.

Table 2: Scope Search Results

\begin{tabular}{|c|c|c|c|c|}
\hline Search Term & Scopus & Science Direct & ProQuest & Google Scholar \\
\hline $\begin{array}{c}\text { "Building Performance } \\
\text { Simulation" AND "Early Stage" }\end{array}$ & 525 & 202 & 47 & 1,180 \\
\hline $\begin{array}{c}\text { "Building Performance } \\
\text { Simulation" AND "Architect" }\end{array}$ & 865 & 340 & 65 & 1,960 \\
\hline "BIM" and "Architect" & 2,028 & 1,074 & 582 & 14,800 \\
\hline "BEM" and "Architect" & 586 & 343 & 248 & 11,700 \\
\hline "IDMM" and "Architect" & 2 & 2 & 84 & 20 \\
\hline \multicolumn{7}{|l|}{} & 4,009 & 1,961 & 1,026 & 29,660 \\
\hline
\end{tabular}

The identification of 36,656 references across four databases shows that there is a large background of information to work off; it also shows that the research topic needs to be refined 
to produce a manageable amount of references. Due to the broad scope of these searches, it is no surprise that many results were found. The bulk of these 36,656 references are from Google Scholar, which locates more "grey" literature than other sources. Grey literature, as identified by ICGL (2010) covers information that is of high enough quality that libraries and databases want to collect and display the information, but no commercial publisher controls the material. Meaning publishing is not the primary focus of the author, this sort of literature is more likely to be scrutinised.

\subsubsection{Focus Idea and Define the Scope}

The ideas presented through an early scope search can be approached from different angles of research. For a determination to be made on which approach was best for the thesis and the expected results, both qualitative and qualitative methods were reviewed. Below are examples of two qualitative and two quantitative approaches to analysing the topic "Simulation of an Architectural model".

Qualitative:

1. How do Architects use an Architectural model to perform BPS?

2. Would Architects use an Architectural model to BEM methodology?

Quantitative:

3. What transfer process is required to allow for Architectural model to BEM?

4. How accurate are BPS of Architectural models?

The qualitative examples focus on the direct effects of an Architect using simulation software. Expected results from this sort of question would be able to lead to further research and an understanding of the behaviour an Architect would have with new specialised software. The first question would almost prompt more questions than it could answer, such as, do Architects use BPS. What BPS is used? Why do Architects use BPS? This sort of question is expected to be common in literature and therefore not useful to be answered for the industry. The second question would need to be answered with a qualitative approach, such as focus groups as seen in the thesis by Braasch (2016).

Simulation based research is often quantitative because numerical results are produced. The questions three and four would both produce numerical results, but they would not be the focus of the research. Question three analyses specific transfer process that is established in the literature. While question four determines, how accurate these approaches are in terms of information translation. 


\subsubsection{Review Question and Inclusion Criteria}

A final review question is shown below and analysed using a PICO (Population, Intervention, Comparator, and Outcome) chart in Table 3. A PICO was used to examine the specifics of the research question relating to "who, what, where, and how". Defining the points, helps ensure that the inclusion criteria for the literature review are sound.

The inclusion criteria are set to ensure that the papers, which make it through, have the attributes that are required. It also allows an analysis to be completed that determines where the review question may be lacking.

Table 3: PICO Chart Analysis

\begin{tabular}{|c|c|c|c|c|}
\hline \multirow{2}{*}{ Research Question } & \multicolumn{4}{|c|}{ Points of Consideration } \\
\hline & Who & What & Where & How \\
\hline $\begin{array}{l}\text { What transfer process is } \\
\text { required to allow } \\
\text { information to be } \\
\text { transferred between an } \\
\text { Architect's model and BEM }\end{array}$ & $\begin{array}{l}\text { Not specified } \\
\text { Needs more } \\
\text { information, } \\
\text { who is using } \\
\text { the process? }\end{array}$ & $\begin{array}{l}\text { Information } \\
\text { transfer between } \\
\text { CAD and BEM } \\
\text { Needs to be clearer } \\
\text { about what is done }\end{array}$ & $\begin{array}{l}\text { Not specified } \\
\text { What setting } \\
\text { is the process } \\
\text { used in? }\end{array}$ & $\begin{array}{l}\text { Not specified } \\
\text { How is it } \\
\text { determined? }\end{array}$ \\
\hline $\begin{array}{l}\text { What transfer process is } \\
\text { required from an Architect's } \\
\text { model so that BEM files can } \\
\text { produce be a reliable } \\
\text { reflection of the potential } \\
\text { performance of the design } \\
\text { ideas? }\end{array}$ & $\begin{array}{l}\text { Not specified } \\
\text { See above }\end{array}$ & $\begin{array}{l}\text { Information } \\
\text { transfer between } \\
\text { CAD and BEM to } \\
\text { allow for reliable } \\
\text { simulations }\end{array}$ & $\begin{array}{l}\text { Not specified } \\
\text { See above }\end{array}$ & $\begin{array}{l}\text { Transfer } \\
\text { process and } \\
\text { results from } \\
\text { energy } \\
\text { simulation } \\
\text { More detail }\end{array}$ \\
\hline $\begin{array}{l}\text { What level of detail, } \\
\text { information, and transfer } \\
\text { process is required for an } \\
\text { Architect's model to } \\
\text { produce a BEM file that can } \\
\text { reliably simulate early } \\
\text { design alternatives in an } \\
\text { Architectural setting? }\end{array}$ & $\begin{array}{l}\text { Architects or } \\
\text { Designers } \\
\text { who produce } \\
\text { CAD models }\end{array}$ & $\begin{array}{l}\text { What level of detail, } \\
\text { information, and } \\
\text { transfer process to } \\
\text { allow for reliable } \\
\text { simulations of BEM } \\
\text { files from CAD }\end{array}$ & $\begin{array}{l}\text { Architectural } \\
\text { setting e.g. } \\
\text { studio or firm }\end{array}$ & $\begin{array}{l}\text { Level of detail, } \\
\text { information, } \\
\text { transfer } \\
\text { method, and } \\
\text { results from } \\
\text { energy } \\
\text { simulation }\end{array}$ \\
\hline
\end{tabular}

\subsubsection{Review Protocol}

For early design feedback to become feasible, Architects need reliable simulations of their concept models and informative results, without having to employ an expert. Ease of use, ease of interpretation, and links with Architectural modelling software are among the required criteria identified from Architects that would allow for effective tool use (Weytjens and Verbeeck, 2010). This can be achieved by understanding what level of detail, information, and transfer process was required to transfer information from an Architectural model. 
Braasch and Donn (2016) show that Architects want, but lack interest in ensuring model accuracy. While Engineers sought accurate concept models, which the Architects could trust and modify to inform their design. This work showed that simulations using VPL are responsive, informative, and accurate enough for Architects and Engineers to use. What was not provided in this work, is a means to link the Architectural model to the advanced simulation programs. Currently, an additional model needs to be produced which may not be as reflective as reality and would need to be updated as each new concept design comes through, consuming valuable time.

Aims of the research question within the literature review are:

- Identify useful background information, which can be used as further evidence for the need for the research

- Identify exactly what Architects want and need in a simulation tool

- Identify processes to simulate environmental conditions from an Architectural model

- Identify software that is used in citations for transfer, simulation, or modelling

The search strategy consists of reviewing online databases using search terms, which are expanded from the research question above. The types of literature that are required must come from reputable sources like conference proceedings, journal articles, or books. Unpublished information would not be used, as it is very unlikely to make it into the search databases. Some of the search terms that can be used are BIM, CAD, and Architect, these and others are strategically sequenced using Boolean logic for each database search. From the final identified papers, information relating to how a transfer process works, how it overcomes known issues, and what programs or file types are being used was extracted. This data was analysed using a measure of success table. These processes are solely carried out by the author of this paper.

\subsection{Database Summary}

Four databases are used within the literature review due to the content they contain.

Table 4: Literature Review Database Summary

\begin{tabular}{|c|l|}
\hline Database & \multicolumn{1}{c|}{ Summary } \\
\hline Google Scholar & $\begin{array}{l}\text { Google Scholar is a search engine for scholarly literature, legal documents, and } \\
\text { patents. It has limited analysis due to mass selecting references not being possible, } \\
\text { better to be used as "grey" literature due to the vast range of content that returns. }\end{array}$ \\
\hline ProQuest & $\begin{array}{l}\text { ProQuest is a multidisciplinary source of scholarly references on topics from } \\
\text { business, social science, health sciences, humanities, and the arts. It provides } \\
\text { citations, abstract, and indexing information. }\end{array}$ \\
\hline Science Direct & $\begin{array}{l}\text { A collection of journals, articles, and book chapters that are peer reviewed within } \\
\text { most science topics. It provides citations, abstract, and indexing information. }\end{array}$ \\
\hline
\end{tabular}




\begin{tabular}{|l|l|}
\hline \multirow{3}{*}{ Scopus } & $\begin{array}{l}\text { Scopus is a multidisciplinary database, which covers peer-reviewed journals in a } \\
\text { vast range of sciences including environmental, social, health, and physical. It } \\
\text { provides citation, bibliographical information including abstracts, keywords, and } \\
\text { funding details. }\end{array}$ \\
\hline
\end{tabular}

\subsubsection{Key Words}

From the research question posed above in section 2.2.4, keywords or ideas can be extracted, which are used in the following literature review. Specific terms may be used to detail what type of software was used in each paper, as these are sometimes used interchangeably, they must all be searched for and identified.

- Building Performance Simulation (BPS), Building Energy Modelling (BEM), Computer Aided Design (CAD), Building Integrated Modelling (BIM)

As this research is aimed at determining a process for simulation in the early stages of design, it is important to identify papers that also follow this methodology. Although, papers that aim to complete something similar at a later design stage or not related specifically to design would not be ignored.

- Design, Analysis, Early-Stage, Concept

Obtaining reference papers that are related to Practitioners in the Architecture Engineering and Construction (AEC) industry would allow for a more robust analysis of what was available.

- Architect, Engineers, Designer, Architecture Engineering and Construction (AEC)

Due to Grasshoppers Visual Programming Language abilities and its direct relationship to simulation tools Honeybee and Ladybug, it cannot be ignored. Finding papers that follow a methodology using this software have a high potential to be helpful and informative.

- Grasshopper, Honeybee, Ladybug

The last term is very broad, it could mean different things under altered contexts, but making it more specific would minimalize results.

- Information Transfer

\subsubsection{Bias}

Bias in identified papers can be a problem if the research question is investigating a question, which could have positive or negative responses. As Boland et al. (2014) establish, a paper can be less likely to be published if negative results are found, this problem can only be mitigated by 
finding a clear overview of the subject from multiple sources including grey literature. They also note that negative results are more likely to be published in smaller local language journals than international English ones.

The research question posed in this review was not subject to the same bias problems due to negative results as negative effects are posed as errors or problems and are written about heavily.

\subsubsection{Results Storage}

Identified results are stored in a bibliographic reference software, this allows for automated storage, search, and duplication deletion. References that are downloaded from the internet and do not contain the full text can be searched for and added. Searching through the references with a search and group function makes it easier.

\subsubsection{Search Results}

Four different sets of search terms are used across the databases. It is assumed that the abbreviated forms of certain words would not be used without the expanded version, but the reverse may be true. Therefore, Building Performance Simulation is used instead of BPS.

1. "Building Performance Simulation" AND “Design" AND "Architect OR Engineers"

2. "Building Performance Simulation" AND "Grasshopper"

3. "Computer Aided Design" AND "Building Energy Modelling"

4. "Computer Aided Design" AND "Building Performance Simulation"

Table 5: Results from Four Different Search Terms

\begin{tabular}{|c|c|c|c|c|}
\hline \multirow{2}{*}{$\begin{array}{c}\text { Search } \\
\text { Term }\end{array}$} & \multicolumn{4}{|c|}{ Results } \\
\cline { 2 - 5 } & Google Scholar & ProQuest & Science Direct & Scopus \\
\hline 1 & 2,590 & 38 & 9 & 42 \\
\hline 2 & 732 & 2 & 2 & 4 \\
\hline 3 & 104 & 1 & 3 & 2 \\
\hline 4 & 747 & 3 & 1 & 11 \\
\hline
\end{tabular}

Google Scholar allows for a small amount of customisation in its searching, patents and citations can be ignored. Searches using ProQuest are done where the keywords are not in the full text of a paper. Science Direct and Scopus both allow the abstract, title, and keywords of each paper to be searched. 


\subsection{Screening Papers}

After conducting the main search, papers are screened for relevance based on inclusion criteria and duplicate references are removed.

\subsubsection{Duplication Removal}

Due to there being identical searches made across different databases, duplicate papers are found. From the searches within ProQuest, Science Direct, and Scopus, 36 duplicates are identified using the bibliographic reference software Endnote.

118 Reverences Identified Through Database Search's
36 Duplicates Identified
82 Unique References Remain

\section{Figure 6: Duplicate Removal from Identified References}

As different databases export slightly different information, duplicate references are merged leading to the maximum amount of information being kept.

\subsubsection{Initial Inclusion Criteria}

Inclusion criteria for identifying useful references are based on the PICO chart established in section 2.2.3, additionally, some more generalised terms are added. Exclusion criteria for papers, which are not relevant, are climate change, student education, case studies, occupant behaviour, green building history, and optimising energy.

Table 6: Inclusion Criteria for References in Study

\begin{tabular}{|c|l|}
\hline PICO Points & \multicolumn{1}{|c|}{ Inclusion Criteria } \\
\hline Who & Architects, Engineers, Designers, AEC Industry, CAD modellers \\
\hline What & $\begin{array}{l}\text { Transfer Method, Level of Detail, Information Transfer, Simulation, Building Information } \\
\text { Modelling, Building Energy Modelling, Computer Aided Design, Building Performance } \\
\text { Simulation }\end{array}$ \\
\hline Where & Architectural Setting, Other \\
\hline How & $\begin{array}{l}\text { The outcome of Transfer Method, Industry Foundation Class Schema, Green Building } \\
\text { XML Schema, Environmental Simulation }\end{array}$ \\
\hline Other & Geometry Generation, Automation, Visualisation, Template Files, Architect Friendly \\
\hline
\end{tabular}

One of the references is excluded at this stage due to not being in English. Then the titles and abstracts of the 82 references are read in relation to the inclusion criteria, 30 references are selected due to the relevant or potentially relevant information. The other 51 references are kept in the reference database in case of future relevance but are essentially removed from the study. 


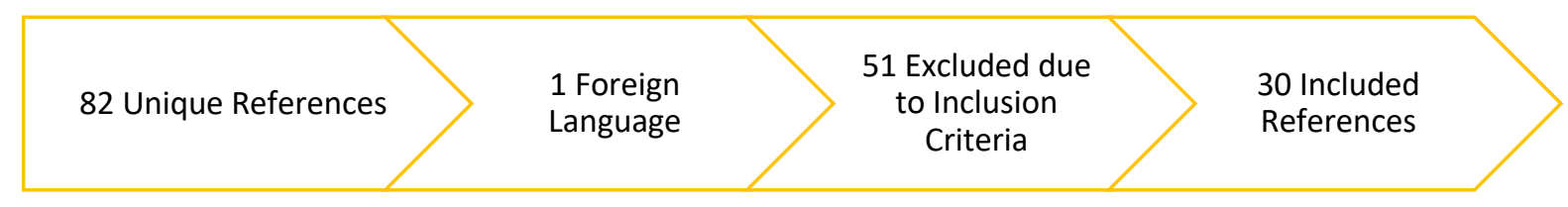

Figure 7: Applying Inclusion Criteria to Remaining References

\subsection{Obtaining Full-Text Papers}

Full-text papers relating to the references have either been downloaded with the database search, downloaded through the reverence managing software's automated search feature, or have been manually searched for and found online. Of the 30 papers, two full texts cannot be found online or in the library. These are excluded from the study, which is a slight limitation.

The obtained papers are read in full and notes are made relating to key information, a summary, methodology, and useful notes. When reading, "snowball" references are also noted down, these are references with relevant information from an identified paper. From the papers, 20 snowballed references are determined. An additional paper is added at this stage due to prior knowledge of its relevance from academics within the university.

30 References $\backslash \begin{gathered}2 \text { Full Texts } \\ \text { Not Avalible }\end{gathered} \quad \begin{gathered}20 \text { Snowball } \\ \text { References }\end{gathered}>\begin{gathered}1 \text { User Added } \\ \text { Paper }\end{gathered}>\begin{gathered}49 \text { Relevant } \\ \text { References }\end{gathered}$

Figure 8: Addition of Snowball References

\subsection{Apply Inclusion Criteria and Select Papers}

The final inclusion criterion to establish the select few papers is that information is being transferred from one data source to another. Nineteen papers are described as doing so, the processes range in the quality of description, method, modelling tool, and simulation tool, leading to almost 19 distinct translation processes.

49 Relevant References

19 Transfer Methods

Figure 9: Identification of Final Transfer Methods

The other 30 papers that have been identified as relevant, but do not contain a specific translation process, are still useful to the research project, because of the background information they establish. Surveys, case studies, and simulation experiments are used primarily as the research methods in these papers. 


\subsubsection{Results}

Database searches identified 118 references that potentially have information relating to the proposed research question. After duplicates and one non-English paper are removed, 82 unique references remain. These are filtered down to 30 papers by using the inclusion and exclusion criteria. Only 28 full-text papers could be found, two are excluded from the study. Reading and understanding the 28 papers identified an additional 20 snowball references, bringing the total identified references to 48 . One user added paper is added due to it being a key part of this thesis. After determining what papers contained information transfer processes another 30 papers are excluded. These papers are still important to the background and need of the thesis work. Having established 19 papers from the initial 118 references, these papers form the current understanding and approach from research. Figure 10 visualises the process.

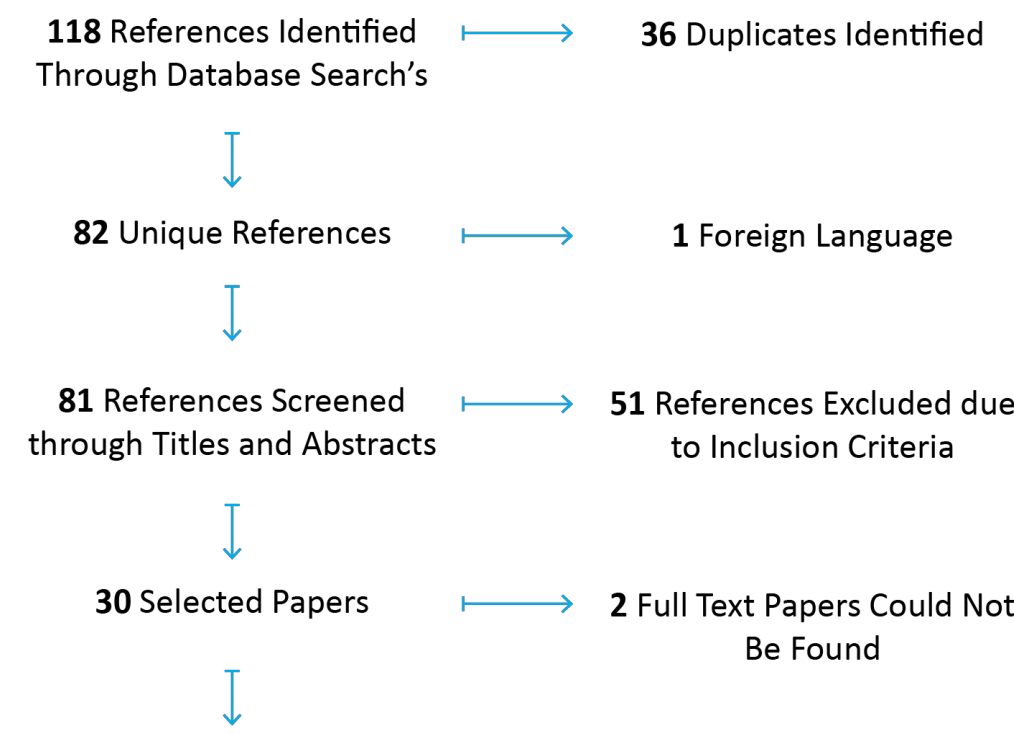

28 Full Text Papers

20 Snowball References

48 Included References

1 User Added Paper

49 Included References

19 Included References

30 Excluded Papers

19 transfer Methods 


\subsection{Assessment of Paper}

Wang and Groat (2013) identify seven research strategies; historical research, qualitative research, correlational research, experimental and quasi-experimental research, simulation research, logical argumentation, and case studies and combined strategies. Of these, only three research strategies have been used over the 49 relevant references identified. As listed in Table 7, 31 of these are simulation, 16 are qualitative, and two are case studies. The 19 identified transfer processes all used simulation research as the research strategy, this is expected, as to test the effectiveness of information transfer, simulations of buildings are inevitably used. A portion of research in the AEC industries would be simulation-based as Wang and Groat (2013) discuss how the strategy is used for simulation in earlier stages of Architectural design processes, integration of simulation software, real-time simulation, immersive building simulation, and modelling construction sequences.

Table 7: Different Research Strategies Identified

\begin{tabular}{|l|c|}
\hline Research Strategy & Amount \\
\hline Simulation Research & 31 \\
\hline Qualitative Research & 16 \\
\hline Case Studies and Combined Strategies & 2 \\
\hline
\end{tabular}

\subsection{Extraction of Data}

Key reference information from the 19 transfer processes were extracted based on performance measures and tabled using the data extraction form shown in the next section 2.8.2. The 30 other identified references do not have specific data to extract into a table, although as they may contain useful information, notes on what results were found are taken.

\subsubsection{Identify the Data to be extracted}

Useful analytical data to be extracted is anything on the outcome of the identified transfer process. Information relating to eight factors is identified as being important. These measures are; manual steps, automated steps, creation program, simulation program, file type, interoperability, workflow, and overall steps. This information is key in determining whether a paper has a viable strategy for information transfer in an Architectural setting. Those eight factors are quantitative, but a final measure is also used which is qualitative. This measure is testing a final factor used to ensure that only papers that can be finished are tested. 
Another set of analytical data which is extracted are the issues the transfer process has, and how the paper overcame known literature established issues such as; thermal zoning, walls and geometry, materials, construction, loads and schedules, location, duplicate data, and missing data.

\subsubsection{Data Extraction Form and Tables}

The data extraction template in Table 8 shows, where and how the information relating to each measure is stored. Questions relating to the requirement are also shown in section 3.2.1. Some of these are determined with a "yes" or "no" question, while the others ask what specific processes or tools are used.

This template is used to identify the key information displayed in Table 10.

Table 8: Example Data Extraction Table

\begin{tabular}{|l|l|}
\hline Reference Paper & Example, $(\mathrm{xxxx})$ \\
\hline Method & $\begin{array}{l}\text { Exports Revit file to Middleware software which converts information into a } \\
\text {.idf file which can be opened and simulated through Energy Plus }\end{array}$ \\
\hline Manual Steps & No \\
\hline Automated Steps & Yes \\
\hline Creation Program & Revit \\
\hline Simulation Program & Energy Plus \\
\hline File Type & Intermediate Data Form (.idf) \\
\hline Interoperability & No \\
\hline Workflow & Yes \\
\hline Overall Steps & 2 \\
\hline Repeatable & Yes \\
\hline
\end{tabular}

\subsubsection{Contacting Experts}

Everyting related to technology is rapidly evolving and changing. It is important to discover what work was underway or had been recently finished but unpublished, to ensure that all current process are identified. Industry experts were contacted directly to find out, 1) Have they done more up to date work, and 2) Can the tools that make their processes work be found anywhere?

Table 9: Responses from Contacting Experts

\begin{tabular}{|c|c|c|}
\hline Expert & Developments? & Tools? \\
\hline 1 & Yes - Another paper was published at the same time & No \\
\hline 2 & Yes - Another paper relating to conversion tools & No \\
\hline 3 & $\begin{array}{r}\text { Yes - A similar process to identified paper, but with more } \\
\text { developed tools that have clash detection }\end{array}$ & $\begin{array}{c}\text { No - Tools are not publicly } \\
\text { available. }\end{array}$ \\
\hline 4 & Yes - A paper with case studies using the transformation \\
algorithm, yet to be published & No & No \\
\hline 5 & & No
\end{tabular}




\begin{tabular}{|c|c|c|}
\hline 6 & No & No \\
\hline 7 & No & No \\
\hline 8 & No & No \\
\hline 9 & No & No \\
\hline 10 & No & No \\
\hline
\end{tabular}

Authors from ten out of the 19 transfer processes identified responded, four answered with "yes", of those, three provided the paper. Only one expert responded about the proprietary tool used and said it is publicly unavailable.

\subsection{Established Translation Processes in Literature}

Each set of translation processes is examined for specifics, from containing automated steps to what file type is used. Where specific software is mentioned they are noted here, if specific software was not used then the category that defined it is used.

Table 10: Assessment of Translation Processes from Identified Literature

\begin{tabular}{|c|c|c|c|c|c|c|c|c|c|}
\hline 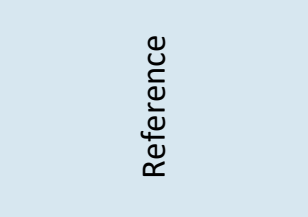 & 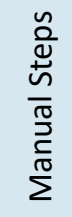 & 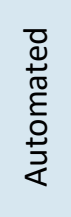 & 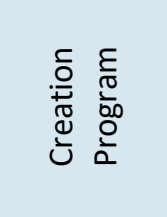 & 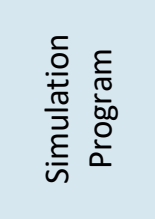 & $\frac{\stackrel{0}{2}}{\frac{0}{i}}$ & 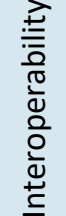 & $\begin{array}{l}\frac{3}{0} \\
\frac{0}{\frac{0}{2}} \\
\frac{2}{5} \\
3\end{array}$ & $\frac{n}{\stackrel{n}{\geq}}$ & $\begin{array}{l}\frac{0}{0} \\
\frac{0}{\pi} \\
\frac{\pi}{0} \\
\frac{0}{0} \\
\frac{0}{0} \\
\simeq\end{array}$ \\
\hline 1 - Kota et al. (2014) & No & Yes & $\begin{array}{c}\text { Yes } \\
\text { (Revit) }\end{array}$ & $\begin{array}{c}\text { No } \\
\text { (DAYSIM) }\end{array}$ & $\begin{array}{c}\text { No } \\
\text { (RAD) }\end{array}$ & No & Yes & 2 & No \\
\hline $\begin{array}{c}2 \text { - Chaisuparasmikul } \\
\text { (2006) }\end{array}$ & Yes & No & $\begin{array}{c}\text { Yes } \\
\text { (Revit) }\end{array}$ & $\begin{array}{c}\text { Yes } \\
(\mathrm{DOE}-2)\end{array}$ & $\begin{array}{l}\text { Yes } \\
\text { (IFC) }\end{array}$ & Yes & Yes & 8 & No \\
\hline $\begin{array}{c}3 \text { - Cerezo et al. } \\
(2014)\end{array}$ & Yes & Yes & $\mathrm{n} / \mathrm{a}$ & $\mathrm{n} / \mathrm{a}$ & $\begin{array}{c}\text { No } \\
(\mathrm{XML})\end{array}$ & No & Yes & $\mathrm{n} / \mathrm{a}$ & No \\
\hline $\begin{array}{c}4 \text { - Yeang and Lam } \\
\text { (2009) }\end{array}$ & ? & ? & $\begin{array}{c}\text { Yes } \\
\text { (Revit) }\end{array}$ & $\begin{array}{c}\text { No } \\
\text { (Insight) }\end{array}$ & $\begin{array}{c}\text { Yes } \\
\text { (gbXML) }\end{array}$ & No & No & ? & No \\
\hline $\begin{array}{l}5 \text { - Kamel and } \\
\text { Memari (2019) }\end{array}$ & Yes & No & $\begin{array}{c}\text { Yes } \\
\text { (Revit) }\end{array}$ & $\begin{array}{l}\text { Yes } \\
\text { (EP) }\end{array}$ & $\begin{array}{c}\text { Yes } \\
\text { (gbXML) }\end{array}$ & No & Yes & 4 & Yes \\
\hline $\begin{array}{c}6 \text { - Santos et al. } \\
\text { (2017) }\end{array}$ & No & Yes & $\begin{array}{c}\text { No } \\
\text { (Rhino) }\end{array}$ & $\begin{array}{l}\text { Yes } \\
\text { (EP) }\end{array}$ & $\mathrm{n} / \mathrm{a}$ & No & Yes & 3 & Yes \\
\hline $\begin{array}{l}7 \text { - Kumar and } \\
\text { Mahdavi (2001) }\end{array}$ & Yes & No & ? & ? & ? & Yes & No & ? & No \\
\hline $\begin{array}{c}8 \text { - Maile et al. } \\
\qquad(2013)\end{array}$ & $\mathrm{n} / \mathrm{a}$ & $\mathrm{n} / \mathrm{a}$ & $\begin{array}{c}\text { No } \\
(C A D)\end{array}$ & $\begin{array}{l}\text { Yes } \\
\text { (EP) }\end{array}$ & $\begin{array}{l}\text { Yes } \\
\text { (IFC) }\end{array}$ & No & No & $\mathrm{n} / \mathrm{a}$ & No \\
\hline $\begin{array}{c}9 \text { - Noack et al. } \\
\text { (2016) }\end{array}$ & Yes & Yes & $\begin{array}{c}\text { Yes } \\
\text { (Revit) }\end{array}$ & $\begin{array}{l}\text { Yes } \\
\text { (EP) }\end{array}$ & $\begin{array}{c}\text { Yes } \\
\text { (gbXML, } \\
\text { IFC) }\end{array}$ & Yes & Yes & 6 & Yes \\
\hline $\begin{array}{l}10 \text { - Pratt et al. } \\
\text { (2012) }\end{array}$ & Yes & No & $\begin{array}{c}\text { No } \\
\text { (3DSMax) }\end{array}$ & $\begin{array}{l}\text { Yes } \\
\text { (EP) }\end{array}$ & $\begin{array}{c}\text { Yes } \\
\text { (gbXML, } \\
\text { IFC) }\end{array}$ & Yes & Yes & 4 & Yes \\
\hline 11 - Bazjanac (2008) & Yes & Yes & No & Yes & Yes & No & Yes & 5 & Yes \\
\hline
\end{tabular}




\begin{tabular}{|c|c|c|c|c|c|c|c|c|c|}
\hline & & & (BIM) & (EP) & (IFC) & & & & \\
\hline $\begin{array}{l}12 \text { - Giannakis et al. } \\
(2015)\end{array}$ & Yes & Yes & $\begin{array}{c}\text { Yes } \\
\text { (Revit) }\end{array}$ & $\begin{array}{l}\text { Yes } \\
\text { (EP) }\end{array}$ & $\begin{array}{l}\text { Yes } \\
\text { (IFC) }\end{array}$ & No & Yes & 5 & Yes \\
\hline $\begin{array}{c}13 \text { - Jeong et al. } \\
(2016)\end{array}$ & Yes & Yes & $\begin{array}{c}\text { Yes } \\
\text { (Revit) }\end{array}$ & $\begin{array}{c}\text { No } \\
\text { (Dymola) }\end{array}$ & $\begin{array}{c}\text { No } \\
\text { (Modelica) }\end{array}$ & No & No & 3 & No \\
\hline $\begin{array}{c}14 \text { - Jones et al. } \\
(2013)\end{array}$ & Yes & Yes & $\begin{array}{c}\text { No } \\
\text { (CAD) }\end{array}$ & $\begin{array}{l}\text { Yes } \\
(E P)\end{array}$ & $\begin{array}{l}\text { Yes } \\
\text { (IFC) }\end{array}$ & No & Yes & 5 & No \\
\hline 15 - Kim et al. (2015) & Yes & Yes & $\begin{array}{c}\text { Yes } \\
\text { (Revit) }\end{array}$ & $\begin{array}{c}\text { No } \\
\text { (Dymola) }\end{array}$ & $\begin{array}{c}\text { No } \\
\text { (Modelica) }\end{array}$ & No & No & 5 & No \\
\hline 16 - Lilis et al. (2017) & Yes & Yes & $\begin{array}{c}\text { Yes } \\
\text { (Revit) }\end{array}$ & $\begin{array}{l}\text { Yes } \\
(E P)\end{array}$ & $\begin{array}{l}\text { Yes } \\
\text { (IFC) }\end{array}$ & No & Yes & 4 & No \\
\hline $\begin{array}{l}17 \text { - Welle et al. } \\
(2011)\end{array}$ & Yes & Yes & $\begin{array}{l}\text { Yes } \\
\text { (DP) }\end{array}$ & $\begin{array}{l}\text { Yes } \\
(E P)\end{array}$ & $\begin{array}{l}\text { Yes } \\
\text { (IFC) }\end{array}$ & No & Yes & 3 & Yes \\
\hline $\begin{array}{c}18 \text { - Ramaji et al. } \\
(2016)\end{array}$ & No & Yes & $\begin{array}{c}\text { Yes } \\
\text { (Revit) }\end{array}$ & $\begin{array}{l}\text { Yes } \\
\text { (OS) }\end{array}$ & $\begin{array}{l}\text { Yes } \\
\text { (IFC) }\end{array}$ & No & Yes & 6 & Yes \\
\hline $\begin{array}{c}19 \text { - Dimitriou et al. } \\
(2016)\end{array}$ & Yes & Yes & $\begin{array}{c}\text { Yes } \\
\text { (Revit) }\end{array}$ & $\begin{array}{l}\text { Yes } \\
\text { (EP) }\end{array}$ & $\begin{array}{c}\text { Yes } \\
\text { (gbXML) }\end{array}$ & No & Yes & 5 & Yes \\
\hline
\end{tabular}

The following sections describe what each reference identified and how the processes within it work. The brief description identifies problems that could mean the tool would not be able to be repeated, or that there is a general lack of information around the subject. If there was enough information to put together a simple workflow of how information is translated it was shown. By describing each process that could be found a broad overview of how translation is performed is illustrated.

\section{1 - Building Information Modelling (BIM)-Based Daylighting Simulation and Analysis}

This paper identifies six processes for translating information from Revit to DAYSIM (Kota et al., 2014). Five of these are existing processes with either four or five steps. The proposed sixth process set out in Figure 11 uses a plugin to Revit called Revit2Radiance, which can complete the process in one-step. Revit2Radiance performs four automated steps; it creates a parameter file to store added information, adds material data to this folder, reads material information into the other software, and translates the Revit model's geometry so that it automatically simulates. Although the process is explained in-depth, the code that performs the steps is not referenced or provided, making testing impractical. The process is shown to work for a test case of a solar decathlon house.

\section{Revit Model}

Revit2Radiance Plugin

Radiance Input File 
2 - Bidirectional Interoperability between CAD and Energy Performance Simulation through Virtual Model System Framework

In this paper from Chaisuparasmikul (2006) a virtual model system is used, which similarly to BIM, facilitates information transfer and integrating for collaborative projects Architectural models are stored on a database that can be drawn from and written to by simulation programs or editors. The virtual model system claims to be able to extract coordinates, geometries, thermal zones, constructions, and material properties from Revit, and translate them into energy analysis files. As a virtual model system is independent of modelling and simulation tools it can be used to store or transfer information. In this example building elements, constructions, and material properties from Revit are directly imported into energy analysis software, allowing for simulation assurance. The process is shown to have benefits in four unique scenarios. As the data-mapping engine of the virtual model system is not shared or expanded upon the approach cannot be tested to the same extent. However, this paper does demonstrate a promising use of the Combined Model Method, which allows for collaborative design and simulation.

\section{3 - Towards Standardised Building Properties Template Files for Early Design Energy Model Generation}

Cerezo et al. (2014) proposes a XML file format structure for storing building properties. The template file is organised into two sections, building templates, and component dependencies. The building template contains general data, constructions, thermal loads, and conditioning systems, of which some information is sourced from the component section. Component dependencies are made up of constructions, materials, and schedules.

\section{4 - Computational Building Performance Modelling and Eco-design}

Yeang and Lam (2009) show the creation of a BPS interface between Revit and Radiance for lighting simulations. The very brief overview paper does not go into any level of depth to explain what file type is used, how translation occurs, or whether the process is interpretable. Therefore, this paper is discarded.

\section{5 - Review of BIM's Application in Energy Simulation: Tools, Issues, and Solutions}

Kamel and Memari (2019) present three processes that are tested on different case studies. Initially Revit to Green Building Studio, then Revit to gbXML to OpenStudio to IDF to EnergyPlus, and last, Revit to Green Building Studio to IDF to EnergyPlus. These processes use a BIM filecorrecting tool that alters the output from the BIM authoring tool. Then an IDF is generated and simulated in energy analysis software. Issues within this process are established and an executable 
code file is developed that corrects Revit's output gbXML file. This code is not provided but it demonstrates an example of what is performed.

\begin{tabular}{|l|l} 
BIM Generating Tool $>$ BIM Export \\
Corrective Tool
\end{tabular}$>$ IDF Generator $>\begin{gathered}\text { Energy Simulation } \\
\text { Tool }\end{gathered}$

Figure 12: Simplified Workflow from Kamel and Memari (2019)

6 - Automation of CAD Models to BEM Models for Performance Based GoalOriented Design Methods

Santos et al. (2017) uses curved Rhino geometry transferred into planar meshes that is simulated using EnergyPlus in Grasshopper. Three different geometries are tested; each is simulated with a genetic algorithm to find an optimal glazing amount. The process in Figure 13 shows that curved surfaces can be simulated using an IDMM method. The process also shows the power of a genetic algorithm to refine a design and lower energy consumption. The paper does not identify a process to transfer geometry from an Architectural model, just that the process once in Rhino/Grasshopper works. This process can be repeated, but it is not based on Architectural modelling software that Architecture firms regularly use.

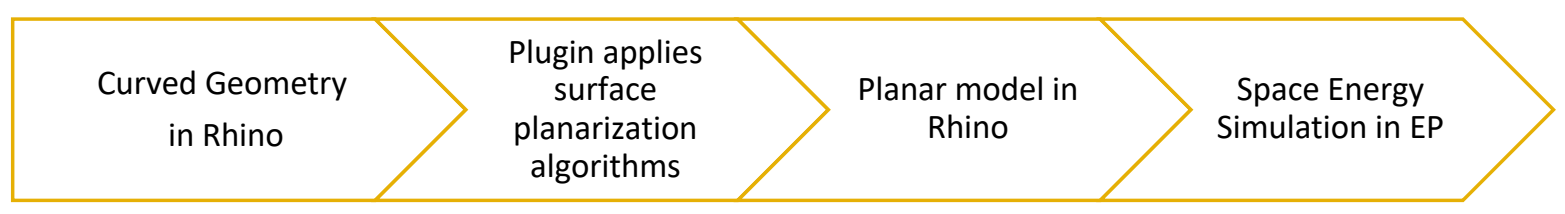

Figure 13: Simplified Workflow from Santos et al. (2017)

7 - Integrating Thermal Comfort Field Data Analysis in a Case-Based Building Simulation Environment

Kumar and Mahdavi (2001) describe and test a thermal comfort module in SEMPER. SEMPER is a computational framework that enables multi-performance measure design. This process provides real-time feedback for Designers and a database of shared object models. From the reference, it is shown that SEMPER is not linked into Architectural modelling software and the process is not explained in enough depth for testing to take place.

\section{8 - BIM-Geometry Modelling Guidelines for Building Energy Performance Simulation}

Maile et al. (2013) determine twenty-one data requirements for comprehensive BPS; these are broken into requirements for heat transfer, solar radiation, and daylighting. This information can be stored in an IFC file type, although there are issues illustrated with this file schema, duplicate objects, incorrect space volumes, missing spaces, missing space boundaries, missing exterior 
walls, alignment of space and building element, incorrect 2nd level space boundaries, incorrect normal vector direction, and geometric tolerances. The established modelling guidelines show that spaces are the most important and require correct geometry and definitions. Spaces must be completely closed; building elements need proper geometry that does not overlap. Building elements need proper material layer sets assigned and modeller's need to check for errors using sections and elevations.

All these problems and guidelines provoke recommendations to enable material property transfer or create specific constructions based on industry standards that already have properties applied. The reference provides a lot of information around the process described and ways that the process can be improved in the future. However, it does not provide or recommend a specific process to transfer information with IFC only that it can be completed.

\section{9 - Technical Challenges and Approaches to Transfer Building Information Models to Building Energy}

Noack et al. (2016) assess proposed approaches from the Design4Energy project. The premise being, a BIM authoring tool such as Revit exports a gbXML into a file checker. This checker embeds additional information into the gbXML where needed. Then a translator software like DesignBuilder or OpenStudio is used too manually output an IDF that can be simulated in EnergyPlus. Another proposed process is to use a gbXML to IDF conversion tool from the Design4Energy project. The final approach uses IFC to transfer data into an energy-extended BIM (eeBIM) data structure using a variety of model manipulation tools (model validator, model combiner, model filtering, and eeBIM2SIM converter). The first two approaches are much less complicated; they require fewer tools and steps to complete. However, the third process allows for the use of specialised tools. This approach provides different processes depending on what file type is used from the BIM tool. There is no validator or conversion tool publicly available to use, which limits the assessment potential. Nevertheless, these processes can be tested without checker or conversion tools by using manual interventions.

\section{0 - Automated Translation of Architectural models for Energy Simulation}

Pratt et al. (2012) determine what the minimal steps for generating a BEM from an Architectural model is, laid out in Figure 14. 


$\left.\left.\left.\left.\begin{array}{|c|c|c|}\text { User interacts with } \\ \text { CAD tool }\end{array}\right\rangle \begin{array}{c}\text { CAD tool } \\ \text { produces } \\ \text { model }\end{array} \quad \begin{array}{c}\text { CAD Plugin } \\ \text { exports } \\ \text { Geomtry } \\ \text { information }\end{array}\right\rangle \begin{array}{c}\text { Model } \\ \text { translator } \\ \text { creates BEM }\end{array}\right\rangle \begin{array}{c}\text { BEM simulated } \\ \text { in BES tool }\end{array}\right\rangle$

Figure 14: Simplified Workflow from Pratt et al. (2012)

This process also aims to work in the reverse direction, allowing automated parametric design optimisation. A list of surface and space rules are determined that the Designer must adhere to if a successful model transfer and simulation is to take place. The surface rules are common to even amateur simulation professionals, such as; surfaces must be modelled as planar, surfaces that form opposite sides of the same wall must be assigned the same construction, or penetrations and openings must be rectangular and fully contained within a parent surface but must be stated for amateurs. The most important information that can be transferred from Architectural modelling software are the surfaces and related vertices. Surfaces can contain any of the following: name, material, layer, number of vertices, and the intersections of each vertex. However, not everything is always transferred. Although there is lacking evidence providing details of the protocols used and how they work. This makes testing of anything other than the generic methodology implausible.

\section{1 - IFC BIM-Based Methodology for Semi-Automated Building Energy Performance Simulation}

A five step methodology is presented in Bazjanac (2008) that allows for semi-automated BPS using IFC based BIM, this is shown below. Full implementation of this methodology was not possible when the paper was written in 2008 due to Heating Ventilation and Air Conditioning (HVAC) data not being transferable.

$\left.\begin{array}{|c|c|c|c|c|}\hline \text { Populate IFC Based } \\ \text { BIM with Data }\end{array} \quad \begin{array}{c}\text { Rule-based } \\ \text { Data } \\ \text { Transformation }\end{array} \quad \begin{array}{c}\text { Rigorous } \\ \text { Model } \\ \text { Checking }\end{array} \quad \begin{array}{c}\text { Building Energy } \\ \text { Performance } \\ \text { Simulation }\end{array}\right\rangle \begin{gathered}\text { Analysis of } \\ \text { Results from } \\ \text { Simulation }\end{gathered}$

Figure 15: Simplified Workflow from Bazjanac (2008)

The methodology is shown to work with EnergyPlus using Middleware tools. The geometry simplification tool and IDF Generator are used to prepare an IFC file to be transformed into an IDF. The geometry simplification tool uses a set of seven rules to transform imbedded data into information readable though EnergyPlus. These rules are; verification of element direction, bypass of space boundaries inside a thermal zone, splitting zone ground slabs into multiple planar slabs, reversing material layers of parallel intersecting elements, transform columns embedded into walls into their own wall definitions, transforming and elements protruding the building volume 
into shading devices. A downside to this approach is that it is an older process that may have outdated information.

\section{2 - A Methodology to Automatically Generate Geometry Inputs for Energy Performance Simulation from IFC BIM Models}

Giannakis et al. (2015) sets out a process to run an energy performance simulation from automatically generated geometry using an IFC file. The complex part of this process is applying a Common Boundary Intersection Projection (CBIP) algorithm that allows second-level space boundaries to be generated. This CBIP algorithm consists of four steps, which aim to convert nonintersecting first level geometry to Boundary Represented Zones. The steps are a data identification stage that classifies what IFC information is required, boundary surface extraction from the IFC, common boundary intersection, and boundary intersection projection. Rules for this process are established for transferring to an IDF in Energy Plus.

\begin{tabular}{|c|c|c|} 
CAD Tool export IFC \\
BIM File
\end{tabular}$\quad \begin{gathered}\text { Validate IFC in } \\
\text { Checker }\end{gathered} \quad \begin{gathered}\text { Apply CBIP } \\
\text { Algorithm }\end{gathered} \quad$ Convert to IDF $>\begin{gathered}\text { Thermal } \\
\text { Simulation in } \\
\text { EP }\end{gathered}$

Figure 16: Simplified Workflow from Giannakis et al. (2015)

This process can obtain and convert second level space boundary information. It contains material property information that is not normally obtainable with a standard IFC export. However, the CBIP algorithm is not provided making testing hard.

\section{3 - A Framework to Integrate Object-Oriented Physical Modelling with Building Information Modelling for Building Thermal Simulation}

The paper by Jeong et al. (2016) uses Object Oriented Physical Modelling to integrate the capabilities of a physical simulation into BIM. The established approach translates BIM to Modelica Building Energy modelling (ModelicaBEM), another simulation language so that thermal simulations can take place. A simplified three-step process for BIM2BEM is proposed, shown in Figure 17.

\begin{tabular}{|l|l|l|}
\hline Create Extended BIM model in Revit & $\begin{array}{l}\text { Execute Revit2Modelica } \\
\bullet \text { Create BIM-Based OOPM } \\
\bullet \text { Run Simulation } \\
\bullet \text { Compot Data } \\
\bullet \text { Assign Zoning }\end{array}\left|\begin{array}{l}\text { Report results back } \\
\text { into Revit } \\
\bullet \text { Generate Topology }\end{array}\right\rangle$ Visualise Information \\
\hline
\end{tabular}


There is no need for schemas such as IFC or gbXML because the process is within Revit like a CoMM. It is achieved by directly accessing the BIM data through Revit's API. Interoperable process may be possible in the future, if information can have an impact on the model using this approach. However, the Revit2Modelica process is explained but not provided. This process would be beneficial if it were to be repeatable.

\section{4 - Automated Translation and Thermal Zoning of Digital Building Models for Energy Analysis}

Jones et al. (2013) describe a five-step process to translate an Architectural model into a BEM using IFC. This workflow uses raytracing to find edges of a parent surface that have not been joint to create a surface boundary. The raytracing process is a complicated set of steps that uses Boolean logic to distinguish what surfaces match. No specific software is mentioned except for "CAD".

$\left.\begin{array}{|c|c|c|}\hline \begin{array}{c}\text { Remove Degenerative } \\ \text { Geomitry from CAD } \\ \text { model }\end{array} & \begin{array}{c}\text { Find boundary } \\ \text { Surfaces } \\ \text { through ray } \\ \text { tracing }\end{array}\end{array} \quad \begin{array}{c}\text { Correct } \\ \text { normals for } \\ \text { each surface }\end{array} \quad \begin{array}{c}\text { Define Space } \\ \text { Boundaries }\end{array}\right\rangle \begin{gathered}\text { Spaces into } \\ \text { BEM Zones }\end{gathered}$

Figure 18: Simplified Workflow from Jones et al. (2013)

The process is validated by creating a BEM file from a complex Architectural model. It uses a simple workflow along with a different process to overcome first level boundary surfaces. The cons of this process are that simulation is not involved, the process for applying raytracing is not determined, and there is no mention of materials or constructions being applied to the new space boundary zones.

\section{5 - Developing a Physical BIM Library for Building Thermal Energy Simulation}

Kim et al. (2015) tests the previously mentioned ModelicaBIM structure on five different complexities of building to determine whether information can be assigned or transferred. Thermal results are simulated and reported on, demonstrating that the process works. This process can be used to convert Revit model attributes into energy model parameters for use in a simulation program. However, the process is not explained in enough detail for reproduction to take place.

16 - Automatic Generation of Second-Level Space Boundary Topology from IFC Geometry Inputs

Lilis et al. (2017) discuss how a CBIP algorithm can be used to generate second-level space boundaries. These would contain more information around boundary conditions and surfaces for zoning. The complex process is shown to work for a building where it has determined the 
differences between the internal air elements, external thermal elements, internal thermal elements, shading devices, and opening elements. The benefits of this process are that IFC models can be manipulated to generate second-level space boundaries, although it is a complex process to repeat.

\section{7 - ThermalOpt: A Methodology for Automated BIM-Based Multidisciplinary}

\section{Thermal Simulation for Use in Optimization Environments}

The process ThermalOpt is described by Welle et al. (2011), it performs automated thermal simulations of BIM based models. Using IFC to transfer data simulations can be completed in EnergyPlus for energy analysis and Radiance for daylighting. The process consists of three steps with ThermalOpt automating additional step in the middle.

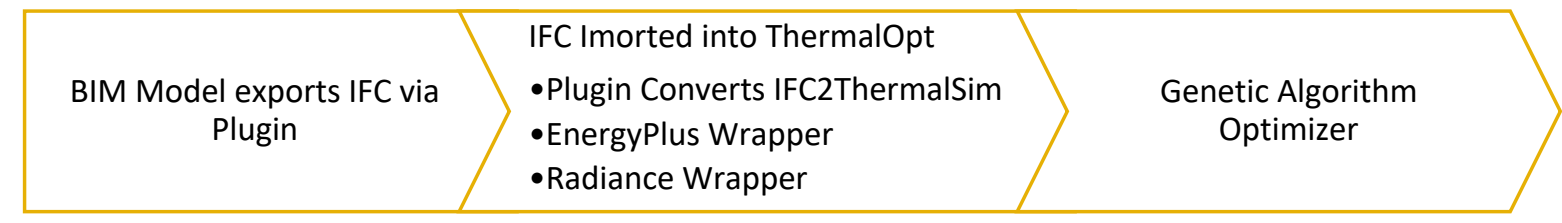

Figure 19: Simplified Workflow from Welle et al. (2011)

Automated processes that are completed in within the ThermalOpt section are explained in-depth throughout the paper as well as in the appendix. Issues with IFC that are identified in other references are also accounted for in this, such as generating second level space boundaries and thermal zones. Industry case studies are conducted that show how the process can be completed and what results are generated. It is discussed that due to the methodology lighting simulations take long periods. An advantage of this process is the demonstration of an optimizer that can be used to provide useful design options based on simulation results. However, the converter and wrappers are not provided by the authors, but they are explained to a high level of depth.

\section{8 - Leveraging Building Information Models in IFC to Perform Energy Analysis in OpenStudio}

In Ramaji et al. (2016) an Architectural model from BIM software is transformed into an OSM file type by BIMserver, before being imported into OpenStudio. Within BIMserver, a steriliser converts IFC information into OSM. The specific sections of information that need to be transferred are described and what they relate to in OSM format, but the steriliser that performs these tasks is not provided. 


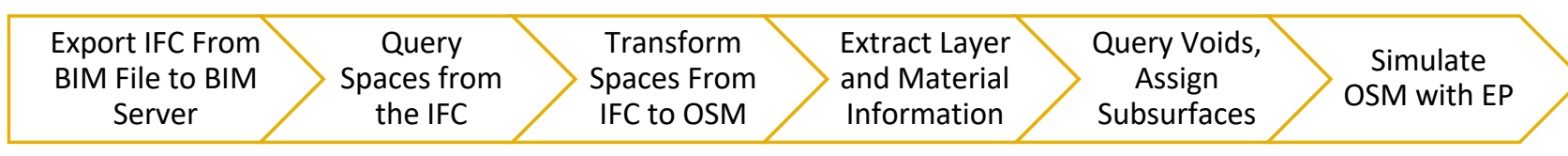

Figure 20: Simplified Workflow from Ramaji et al. (2016)

This process is almost a full-automated process if BIMserver is involved, but only energy analysis can be run.

\section{9 - BIM Enabled Building Energy Modelling: Development and Verification of a gbXML to IDF Conversion Method}

The paper by Dimitriou et al. (2016) starts with building an Architectural model and exporting a gbXML file. This file is corrected by applying editing instructions to the original gbXML file. After missing information has been added the gbXML, it is converted to IDF, which can be run in EnergyPlus.

$\left.\left.\begin{array}{|c|c|c|}\hline \begin{array}{c}\text { Construct Revit Model } \\ \text { and Export gbXML }\end{array} & \begin{array}{c}\text { Identify } \\ \text { Missing } \\ \text { Information }\end{array} & \begin{array}{c}\text { Editing Tool to } \\ \text { apply Missing } \\ \text { Information }\end{array}\end{array}\right\rangle \begin{array}{c}\text { Convert } \\ \text { gbXML to IDF }\end{array}\right\rangle \begin{gathered}\text { Run EP } \\ \text { Simulation }\end{gathered}$

Figure 21: Simplified Workflow from Dimitriou et al. (2016)

The process is completed and shown to be reliable. However, the gbXML editing tool and the gbXML to IDF conversion tool is not shown or available for use, as well as IDF's can only be used for energy simulations the process is not automated and there is no back transfer. 
Chapter Two: Literature Review 
Chapter Two: Literature Review 


\section{Chapter Three: Methodology}

In the previous chapters, the problems with current BPS implementation and the need for proper translation was established. Processes to link Architectural models to BEM were found, but it is unknown:

- Whether any of them currently work to facilitate ready modelling of building performance during sketch design, as well as during developed design?

- Whether there are any common problems or more successful approaches that might form the basis of future improvements in the way, Architect's and consultant's models work together?

This chapter presents a methodology for producing data from which conclusions about the research questions above were raised. Testing allows for the identification of which processes currently work and if there is a method to make them work. In addition, through testing, common problems or approaches that are more successful were highlighted. Two different models are used to perform the tests. A low complexity model to ensure a translation process could be viable, and then a higher complexity model that is more like an Architectural model to identify failures or successes. If the low complexity fails, adjustments can be made until a successfully process. This is to ensure when the complex model is simulated, any issues produced can be related to a flaw in the process.

The scenario set in Chapter One can now be developed due to translation processes being identified. However, there are many unknown parts of the processes including BPS requirements, current problems and issues, model complexities, modelling processes, and different file schemas. Each of these areas is likely to influence information translation. 


\subsection{Approach}

Braasch 2016, implemented energy modelling into a Visual Programming Language (VPL) and demonstrated the benefits to Architectural and Engineering Practitioners using a process that enables them to test multiple building environments off a single model. This work highlights the possibilities of BPS, however, the limitations of having to re-model designs constantly throughout the concept and development design outweighs these benefits. Crude or simple Architectural models can currently be translated via a file schema to run in different environments than they were originally intended for, and models that are more complex can be translated by specialists for specific means (Noack et al., 2016, Kota et al., 2014, Pratt et al., 2012). As the AEC industry is heading ever closer to a one model BIM approach, the benefits of storing all information in once place rather than scattered across fragmented models have been proven (Ganda, 2019). This allows the question to be proposed, why is there no simple means to translate building information from an Architect's model to BEM for analysis. If solved, many quick and simple building simulations would be able to take place during the building development life cycle. The results inform Designer and Engineers about what actions should be taken so that comfort and the overall sustainability of the design can increase.

The literature identified in Chapter Two was condensed through a systematic process down to several relevant translation methods. These 19 references each contain an approach to obtaining building geometry within a BEM environment. In this chapter, an in-depth review identifies how each approach handles categorised issues. Compiling this information allows for the identification of generalised approaches, common problems, and how issues are avoided, in addition to showing which processes do not integrate the required information.

A set of measures of success criteria is established from how issues and errors could occur and used across the identified translation processes to highlight the most successful. Research by Alsaadani and Bleil De Souza (2016) shows that Architects want BPS to link with their Architectural modelling software, but also don't want to compromise on design ability (Weytjens and Verbeeck, 2010). Through using the measures highlighted in the literature as being beneficial to Architects, the ranking process in references can be solely based on their needs. To rank the processes effectively, criteria of what a good process would contain is required. The method for accessing processes is to identify the important measures and state that if all of these were meet, a process would achieve a ranking of $100 \%$. This allows ranking as process with higher scores would be a more optimal approach. The measures are each given a percentage out of 100 to rank how beneficial it appears in literature. Measures that are deemed through the literature to be required 
for Architects to conduct BPS are given higher ratings. Applying the measures of success allows the highest ranked references to be tested, so that where and how issues occur is identified.

The five highest ranked processes are tested through testing a process with a set geometrical and analytical model. These problems are ones that an Architect or an inexperienced person from the AEC industry would experience if they used the translation process.

To test processes two building models were constructed. A simple model is initially used to ensure geometric information, basic information, and that the translation process is possible. A successful process means a more complex model is used after. The complex model determines exactly what types of information are translated, as it contains all the required information in the original Architectural model. If either model fails due to an identified or unidentified issue, what causes the failure is noted. This can then be manually adjusted to work so that other issues can be identified. Working through identified translation processes provides a deeper understanding of why specific ones fail and where the issues originate. Two models are sufficient enough to identify if process work, the issues, and the successes. The only other model that could be used would be an Architectural model from a design firm. However, there would be little benefit from that as the high complexity model covers all the complexity of that model.

\subsection{Rating of Translation Processes}

As discussed in section 2.4.2 the initial inclusion criteria determined what information was desired to be in successful studies. These essentially regulate the characteristics of the included studies. A maximum achieving study contains a fully automated process to extract certain information required for BEM simulation from a much larger Architectural model, and translate this information into the relevant simulation software. Through determining how to rate each process, future approaches can also be assessed in the same way. Aiming the rating process towards Architects helps allow for reliable simulations during early design alternatives in an Architectural setting.

\subsubsection{Measures of Success}

Translation processes were evaluated against eight measures of success. These measures are important aspects of an ideal Architectural model to BEM translation (Weytjens and Verbeeck, 2010, Attia et al., 2012, Maile et al., 2013, Soebarto et al., 2015, Kamel and Memari, 2019). Rating each translation process against all the measures allows for a systematic list from most beneficial to least. The following measures are described and rated as a percentage out of 100 . This is based on how beneficial the measure is according to examples in literature. 


\section{Measure One \& Two}

Measures one and two are important because Weytjens and Verbeeck (2010) found from interviewing Architects, that the highest criterion for an energy evaluation tool is the ease of use. Reducing the number of manual tasks, allows an easier workflow to be understood and used by Architects. An automated process can be any of the steps if the user does not have to undertake them.

These measures are used to establish how much manual manipulation is required and if processes can be automated. Automated steps are seen to be more beneficial to a future program than manual steps, as it enables time to be saved, a reduction in human error, and lower costs (Kamel and Memari, 2019). 18.75 percentage points are awarded if the transfer process contains any sort of automated step that has been implemented to remove human interaction, if this is not true then "no" is answered which is awarded zero points. For the manual step measure, opposite reasoning is applied, meaning if manual steps are used, zero points are awarded, and if not, then $12.5 \%$ is awarded. A manual step is anything that requires data input, or information to be transferred from one source to another by a user. From just these two measures, three different outputs can be achieved; zero percent where only manual steps are used, $18.75 \%$, where both manual and automated steps are used, and $31.25 \%$ when only automated steps are used.

As automation is more desirable in BPS, it necessitates a higher percentage rating, meanwhile the manual steps are not portrayed as desirable in the literature, so are given a neutral weighting (Attia et al., 2012). A manual step is something that a user must actively do, like interact with software using a keyboard or mouse e.g. start a simulation, compile results, find errors, import information, or export information.

\section{Measure Three \& Four}

Creation and simulation program measures are used to determine what software Architectural models are created in and what software the BPS takes place in. A rating of $12.5 \%$ is achieved when common industry-standard software is used. This is to ensure that when testing takes place, processes can be repeated to the detail required. Zero percent is awarded if the software is not commonplace in an Architecture firm, or just classified as Architectural model software, BIM, BPS, or BEM. Due to the unknowing nature of what has been used, which does not help the process of determining beneficial features or processes. Soebarto et al. (2015) identify that a barrier for Architects to perform BPS analysis is that significant investment would be needed in tools or software. By incorporating modelling software, that Architects may already own and be familiar with into a process only enhances its chances of being used and providing a benefit. Additionally, 
the same paper ascertains that consultants are used $14 \%$ of the time due to software cost being too high, and $23 \%$ of the time due to there being no specialist in the office.

Model creation programs used in Architectural practice include but are not limited to SketchUp, AutoCAD, Rhino, Microstation, ArchiCAD, Revit, and 3DSMax. The most common of these are identified as SketchUp, AutoCAD, and Revit (Soebarto et al., 2015). While SketchUp and AutoCAD are in the highest quantity used for design, they do not have an ability to apply in-depth information as ArchiCAD and Revit allow. Therefore, $12.5 \%$ is only achieved if the model is created in ArchiCAD or Revit.

Ten BPS programs compared in Attia et al. (2009) are used to define the fourth measure. This paper identifies which program within the AEC industry is the most "Architect Friendly" based on two factors. The ten programs are; Energy Plus, OpenStudio, Green building Studio, DOE-2, eQuest, IES VE, ECOTECT, HEED, Energy 10, Design Builder. If one of these programs is used as the simulation software in an identified reference, $12.5 \%$ is awarded.

Measure three and four are set at an average level due to there being more important, in addition to less important measures. For an Architect, there are only minor differences between features the model creation programs provide, which mean there are no major advantages of one over another. However, for BPS, certain programs allow more information to be applied and exported than others. The simulation programs differ more, but overall complete a similar task of energy or environmental simulation, justifying a similar rating.

\section{Measure Five}

The file schema used to record translated building information from an Architectural model is important due to each schema having the ability to contain different levels of information (Kamel and Memari, 2019). If a common file type like IFC or gbXML is used, $6.25 \%$ is awarded. However, for any other file type like IDF, OSM, or XML zero percent is awarded, because these file types are not designed for interoperability. This discrepancy is because Architectural modelling software can only export quality models in certain formats, and although IDF and OSM are energy specific, the capability is not there to write to these schemas.

The percentage for this measure is lower due to many of the transfer processes identified already using one of the common file types. Additionally, there is a difference in how IFC and gbXML store and use information; this can be seen in section 3.7.1 and 3.7.2. 


\section{Measure Six}

Interoperability is determined if model information can be transferred back to the creation program after simulations have taken place. In an ideal workflow, all the relevant information along with an updated model would be transferred; however, none of the identified references performs interoperability at this level. For a reference to be awarded $18.75 \%$, any level of information must be transferable and have an impact of the original model. If this is not met, the process receives zero percent.

This measure is set at a higher percentage due to the small number of references that highlight interoperable operations. Attia et al. (2012) determine this to be one of the five criteria selection criteria, while Soebarto et al. (2015) highlight its needed in order for Architects to be able to perform BPS.

\section{Measure Seven}

Process seven assesses whether the processes are explained visually or in writing to a reasonable level of detail. If the basic information of a translation processes is explained, $6.25 \%$ is awarded. If there is no explanation, or the level of detail is low, zero percent is awarded. The process is given a lower percentage due to the potential to enhance testing in later chapters.

\section{Measure Eight}

The steps measure is used to determine and rate how many steps the process takes from the initial program to having simulating results. A length of a workflow can determine its viability, as shorter processes have a lower chance of errors or loss of information. A highly efficient process would only take one-step, e.g. export relevant information into a simulation program, and run.

Information loss is identified in the literature as an issue, the measure awards $12.5 \%$ if there are no more than one file translations in which information could be lost (Welle et al., 2011, Maile et al., 2013, Giannakis et al., 2015, Lilis et al., 2017). Information can be lost anytime the schema changes and compromises on what can be stored are made.

\subsubsection{Complete Rating}

Table 11 illustrates the measures of success that are applied to each translation processes. The requirement statement is used to access which rating score is applied. The previous sections explain each measure in depth; discuss why it is needed, and how it is used.

Table 11: Ranking Requirements for the Measures of Success

\begin{tabular}{|c|l|c|}
\hline Measure & \multicolumn{1}{|c|}{ Requirement } & Rating \\
\hline One & Are manual steps involved in the transfer process? & Yes: $0 \%$ \\
\hline
\end{tabular}




\begin{tabular}{|c|c|c|}
\hline Manual Steps & & No: $12.5 \%$ \\
\hline $\begin{array}{c}\text { Two } \\
\text { Automated Steps }\end{array}$ & Are automated steps involved in the transfer process? & $\begin{array}{l}\text { Yes: } 18.75 \% \\
\text { No: } 0 \%\end{array}$ \\
\hline $\begin{array}{c}\text { Three } \\
\text { Creation Program }\end{array}$ & $\begin{array}{l}\text { Are models constructed in commonly used Architectural } \\
\text { software? e.g. ArchiCAD, Revit }\end{array}$ & $\begin{array}{c}\text { Yes: } 12.5 \% \\
\text { No: } 0 \%\end{array}$ \\
\hline $\begin{array}{c}\text { Four } \\
\text { Simulation Program }\end{array}$ & $\begin{array}{l}\text { Are Simulations completed using common BPS software? e.g. } \\
\text { EnergyPlus, OpenStudio }\end{array}$ & $\begin{array}{c}\text { Yes: } 12.5 \% \\
\text { No: } 0 \%\end{array}$ \\
\hline $\begin{array}{c}\text { Five } \\
\text { File Type }\end{array}$ & $\begin{array}{l}\text { Is IFC or gbXML used as the file schema to transfer } \\
\text { information? }\end{array}$ & $\begin{array}{c}\text { Yes: } 6.25 \% \\
\text { No: } 0 \%\end{array}$ \\
\hline $\begin{array}{l}\text { Six } \\
\text { Interoperability }\end{array}$ & $\begin{array}{l}\text { Are simulation results transferred back to Architectural } \\
\text { modelling software automatically? }\end{array}$ & $\begin{array}{l}\text { Yes: } 18.75 \% \\
\text { No: } 0 \%\end{array}$ \\
\hline $\begin{array}{c}\text { Seven } \\
\text { Workflow }\end{array}$ & $\begin{array}{l}\text { Is the workflow shown or described at a reasonable level of } \\
\text { detail? }\end{array}$ & $\begin{array}{l}\text { Yes: } 6.25 \% \\
\text { No: } 0 \%\end{array}$ \\
\hline $\begin{array}{c}\text { Eight } \\
\text { Transformation Steps }\end{array}$ & $\begin{array}{l}\text { Is there no more than one file transformation where } \\
\text { information could be lost? }\end{array}$ & $\begin{array}{c}\text { Yes: } 12.5 \% \\
\text { No: } 0 \%\end{array}$ \\
\hline
\end{tabular}

\subsubsection{Final Selection Criteria}

To ensure the reference is explained with enough depth for testing to take place each transfer processes is evaluated against the final measure in Table 12. As part of the research question is to determine what errors and issues occur, in addition to if Architects can use the processes, ensuring that there is enough information for the testing to take place is used as a final quality assessment step. Some processes refer to programs, code, or steps that have not been provided. Therefore, testing cannot be completed. The top five translation processes are evaluated and it is determined whether each can proceed based on the requirements set in Table 13. Any disregarded translation processes are replaced with the next highest scoring reference.

Table 12: Testing Measure

\begin{tabular}{|c|l|c|}
\hline Measure & \multicolumn{1}{|c|}{ Requirement } & Rating \\
\hline Nine & Is there enough information for testing to take place? & Yes: Proceed \\
Testing & No: Disregard \\
\hline
\end{tabular}

A translation process provides enough information to proceed if it is not too complex, focuses on the correct areas of research, and explains what programs are used to get to the outcome established.

Table 13: Testing Criteria

\begin{tabular}{|l|c|}
\hline Criteria & Process \\
\hline $\begin{array}{l}\text { A complex process that requires more information than is available during } \\
\text { early design stages }\end{array}$ & Disregard \\
\hline $\begin{array}{l}\text { Information such as code, software, executables, or plugins that are not } \\
\text { publicly available, and there is no workaround known }\end{array}$ & Disregard \\
\hline
\end{tabular}




\subsection{Information Requirements for Building Simulation}

Understanding what information a building simulation requires to run successfully can help control which information needs to be implemented at the start of the modelling process. In other words, understanding what is needed at the end, informs the start. The refined Integrated Dynamic Modelling Method shown in Section 1.2, allows Engineers to work with Architects at the start of a project, this can ensure the correct information and trust of quality at the end. The basic inputs required to run a generic simulation of a certain type are described below.

\subsubsection{Energy Simulation}

Energy simulation in software such as EnergyPlus requires a large amount of different building information to run successfully (LBNL, 2018). It requires:

- Geometry

- Material properties

- Constructions

- Building loads and schedules

- HVAC system or ideal loads

- Weather data

The geometry required must be 3D and created with 2D planar surfaces. All edges need to be connected for the programs to calculate internal volumes and determine where spaces lie. Any subsurface within this geometry resembling windows or doors must also be identified correctly for a program to understand they are joined to a parent surface.

Material properties are required so that geometry can have a calculable effect on the temperature of the building, which energy consumption is calculated from. The specific properties that are required are roughness, conductivity, density, specific heat capacity, thermal Absorptance, solar Absorptance, and visible Absorptance.

Elemental constructions are built up from the materials described above, to form walls, floors, roofs, ceilings, windows, doors, and more. Without constructions, there is no way to apply materials to geometry.

Building loads such as wattage from lighting, computers, and appliances are required to generate consistent internal heat within a space. These loads additionally require schedules to be run off, which allows loads to differ over a period. Schedules are set from daily, all the way to yearly, and 
are not just applied to loads. They can also be used to determine the heating and cooling set points for the HVAC system.

An HVAC system or ideal air loads need to be set and they determine the efficiency of heating and cooling a space. Typically, an HVAC system has higher efficiency, but this can only be used if an actual system is known. If not, ideal loads are used that assume a low efficiency of 1:1.

Weather data and location information are required to place a simulation in a realistic location. Without this, a simulation would not know all sorts of factors such as solar radiation, wind velocity, or external temperatures.

Other factors that are required to run correctly in an energy simulation are zone identification, surface boundaries, and shading surfaces. Each of these mechanisms adds to the realism and accuracy of the simulation.

\subsubsection{Thermal Simulation}

Thermal simulations can either be related to energy simulations and calculate the temperature via heat losses and gains, or it can be a 2D heat flow analysis. At a minimum, thermal simulation requires:

- 2D planar section details

- Material properties

The same properties are required for thermal simulation as thermal simulations. However, to conduct $2 \mathrm{D}$ analyses, sections with each material identified are required. Along with an identification of where the inside and outside surface are located. Internal and external temperatures are required to visualise Heat flow or thermal bridge analysis. This form of simulation can be conducted in THERM or the THERM plugin to Grasshopper.

\subsubsection{Lighting Simulation}

Light simulations can be of either natural sunlight or artificial electric lighting, and they are measured and calculated differently.

- 3D geometry created with 2D planar surfaces and wall thickness

- Material Properties - Reflectance, Roughness, Secularity

- Weather Data

The geometry required is like that needed for energy simulations apart from the need to model wall thickness. Because the sill and edges of windows can shade and reflect light deeper into a 
room they are important for daylight modelling. Whereas, for artificial light internal objects are more important to have modelled.

Different material properties are required for each surface, these being reflectance, roughness, and secularity. As with thermal, weather data is required for daylight simulations.

\subsection{Translation Issues}

Simulation failure can be caused by issues in the model, translation, or analysis software. Issues caused by Architectural modelling or BEM software cannot be fixed without a developer's input. Any issues or errors that occur during simulation would be out of an Architect's range of competence to solve. Therefore, they would be less likely to conduct BPS to avoid them occurring (Weytjens and Verbeeck, 2010). Understanding what can occur strengthens the potential to solve the issues, to allow Architects to use BPS with confidence.

\subsubsection{Review of Potential Issues}

A range of different issues can arise when converting an Architectural model into a transferable file type. It does not matter whether gbXML or IFC is used, similar problems relating to the geometry, element properties, and simulation properties occur, although some schemas can be worse for specific tasks. Kamel and Memari (2019) identify characteristics of gbXML and IFC in a comparative table; this is shown in Table 14. The schemas are not interchangeable because the data structure is quite different and they both take different approaches.

Table 14: Comparison of gbXML and IFC (Kamel and Memari, 2019)

\begin{tabular}{|c|c|c|}
\hline Characteristic & gbXML & IFC \\
\hline Building Geometry & Only rectangular planar geometry & IFC, PKZIP, and XML \\
\hline Data Structure & $\begin{array}{c}\text { XML (eXtendible Mark-up } \\
\text { Language) }\end{array}$ & Bottom-up approach \\
\hline Data Structure Approach & Top-down approach & Yes \\
\hline $\begin{array}{c}\text { Domain of Application } \\
\text { Capability of Defining } \\
\text { Thermal Zones }\end{array}$ & Mostly energy simulation & $\begin{array}{c}\text { Different domains such as building } \\
\text { construction to building operation }\end{array}$ \\
\hline Location & Yes & No \\
\hline Standard Content Set & No & Yes \\
\hline Material Thickness & Yes & Yes \\
\hline $\begin{array}{c}\text { Limited Data Related to } \\
\text { HVAC System }\end{array}$ & Yes & Model View Definition \\
\hline \multicolumn{2}{|c|}{} & \multicolumn{2}{c|}{} \\
\hline
\end{tabular}

Dong et al. (2007) conclude that gbXML is simpler and easier to understand than IFC due to its bottom-up approach and that IFC may have potential benefits due to the comprehensive top- 
down approach. Jalaei and Jrade (2014) identify that gbXML has a bottom-up approach, that is simple and open source. This schema can result in smaller files but also lacks some information. The top-down approach in IFC is complex and has a large file size due to backtracking information at each step. It should also be identified that Kamel and Memari (2019) have misrepresented Jalaei and Jrade (2014) definition of gbXML and IFC data structure.

Table 14 highlights some of the characteristics of each schema, though it does not provide insight into the problems. Jalaei and Jrade (2014) found that sometimes data being transferred to an IFC file from an Architectural model might be missing in the output file, this included information about constructions, loads, and location. The paper by Maile et al. (2013) report on case studies of buildings that are used to illustrate problems relating to many IFC transfers, the problems that arose are tabled below along with problems from other sources.

Table 15: Identified Problems with IFC

\begin{tabular}{|c|c|c|}
\hline Problem & Description & Identified From \\
\hline Constructions & $\begin{array}{l}\text { Missing construction properties (R-value, Conductivity, } \\
\text { Thermal Mass) }\end{array}$ & (Jalaei and Jrade, 2014) \\
\hline Loads & $\begin{array}{l}\text { Missing loads (Occupancy, Lighting, Equipment), either } \\
\text { not applied in BIM or not transferred }\end{array}$ & $\begin{array}{l}\text { (Jalaei and Jrade, 2014) } \\
\text { (Kim et al., 2015) }\end{array}$ \\
\hline Location & $\begin{array}{l}\text { Missing location information such as longitude and } \\
\text { latitude or weather file }\end{array}$ & (Jalaei and Jrade, 2014) \\
\hline Geometry & $\begin{array}{l}\text { Geometry objects duplicating by being defined twice in } \\
\text { the same location }\end{array}$ & (Maile et al., 2013) \\
\hline Spaces & Missing space objects & $\begin{array}{l}\text { (Maile et al., 2013) } \\
\text { (Kim et al., 2015) }\end{array}$ \\
\hline $\begin{array}{c}\text { Space } \\
\text { Boundaries }\end{array}$ & $\begin{array}{l}\text { Missing space boundaries occur when elements do not } \\
\text { properly align or being defined twice }\end{array}$ & (Maile et al., 2013) \\
\hline Exterior Walls & Missing walls causes spaces to not form & (Maile et al., 2013) \\
\hline $\begin{array}{l}\text { Building Element } \\
\text { Location }\end{array}$ & $\begin{array}{l}\text { Misalignment of space and building element causes } \\
\text { incorrect space boundaries to be generated }\end{array}$ & (Maile et al., 2013) \\
\hline $\begin{array}{l}2^{\text {nd }} \text { Level Space } \\
\text { Boundaries }\end{array}$ & $\begin{array}{l}\text { 2LSB are incorrectly or not generated with some } \\
\text { geometry }\end{array}$ & $\begin{array}{l}\text { (Maile et al., 2013) } \\
\text { (Lilis et al., 2017) }\end{array}$ \\
\hline Vector Direction & $\begin{array}{l}\text { Incorrect normal vector direction means geometry will } \\
\text { be facing the incorrect direction (Inside out) }\end{array}$ & (Maile et al., 2013) \\
\hline $\begin{array}{l}\text { Geometric } \\
\text { Tolerance }\end{array}$ & $\begin{array}{l}\text { Geometry will not "snap" due to a small tolerance } \\
\text { causing gaps to form }\end{array}$ & (Maile et al., 2013) \\
\hline Rooms/Zones & $\begin{array}{l}\text { An Architectural model will be zoned by rooms, not by } \\
\text { thermal zones which are required for an energy } \\
\text { simulation }\end{array}$ & (Kim et al., 2015) \\
\hline Wall Thickness & $\begin{array}{l}\text { Thermal zones can generate with a gap between them } \\
\text { due to wall thicknesses duplicating }\end{array}$ & $\begin{array}{c}\text { (Toth et al., 2011) } \\
\text { (Ramaji et al., 2016) }\end{array}$ \\
\hline
\end{tabular}


Many of the issues with the IFC schema being used in file translation are identified from multiple sources (Jalaei and Jrade, 2014, Kim et al., 2015, Maile et al., 2013, Lilis et al., 2017, Toth et al., 2011, Ramaji et al., 2016). These issues are simpler to identify when they occur due to them occurring during simulation, like missing zones or geometry. Other issues such as vector direction or geometric tolerance are harder to determine. IFC is more comprehensive than other schemas because it is about generic translation. It therefore might be expected to have more problems with a specific task like Architectural model to BEM than a dedicated translator would. Problems that have been identified to occur with gbXML file types are tabled below, although, there are less in quantity the importance is relatively high.

Table 16: Identified Problems with gbXML

\begin{tabular}{|c|l|c|}
\hline Problem & \multicolumn{1}{|c|}{ Description } & Identified By \\
\hline Shading & Incorrect shading surfaces & (Lilis et al., 2017) \\
\hline Geometry & Missing wall geometry & (Lilis et al., 2017) \\
\hline Wall Thickness & $\begin{array}{l}\text { Thermal zones can generate with a gap between them } \\
\text { due to wall thicknesses }\end{array}$ & (Toth et al., 2011) \\
\hline
\end{tabular}

Lilis et al. (2017) determines that of the two schema discussed, IFC is more suitable for information translation due to it being richer in content and having the ability to be interoperable. Ramaji et al. (2016) state that IFC models are better for information exchange as gbXML cannot capture all the information and relationships contained in a BIM. Additionally, IFC is more rich data models that can handle bi-directional exchanges of information. These two papers make valid points; however, the amount of issues identified with IFC may not outweigh its potential benefits of more information and interoperability.

All the established issues contain some common ground between them and can be categorised into eight areas.

\subsubsection{Issues Identified from Literature Review}

The problems and issues identified in the section above were sorted into eight different categories shown in Table 17.

Table 17: Assessment of Categorised Issues

\begin{tabular}{|l|l|}
\hline Issue & Assessment \\
\hline Thermal Zoning & Are thermal zones used? If so, what software are they constructed in? \\
\hline Walls and Geometry & Are walls or geometry created? If so, in what dimension and what software? \\
\hline Materials & Are materials used? If so, applied or transferred from what software? \\
\hline Construction & Are constructions used? If so, applied or transferred from what software? \\
\hline Loads and Schedules & Are loads or schedules used? If so, applied or transferred from what software? \\
\hline
\end{tabular}




\begin{tabular}{|l|l|}
\hline Location & Are locational data used? If so, where is it from? \\
\hline Duplicate Data & Are data duplication problems identified in the workflow? \\
\hline Missing Data & Are missing data problems identified in the workflow? \\
\hline
\end{tabular}

The categories above are used with the assessment statement next to it to determine what issues are present in the identified references. The questions aim to conclude if an issue is dealt with, and in what way. The second category, walls, and geometry, contains three levels of information gathering. It aims to highlight; what dimension walls are translated in as this can have an effect of different simulation environments. Table 18 shows the results of this information. Interestingly, only one reference has the potential to contain all eight issues categorised, while the average amount of issues is over $50 \%$ at $4.7 / 8$.

Table 18: Categories of Potential Issues

\begin{tabular}{|c|c|c|c|c|c|c|c|c|}
\hline 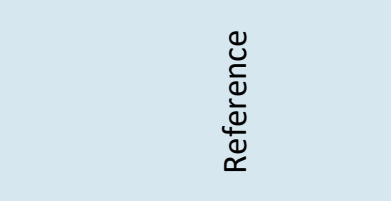 & 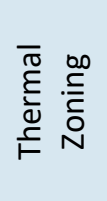 & 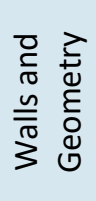 & 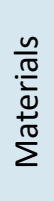 & 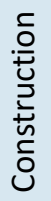 & 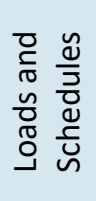 & 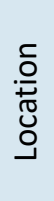 & 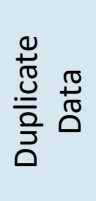 & 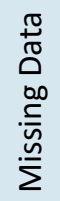 \\
\hline \multicolumn{9}{|l|}{1 - Kota et al. (2014) } \\
\hline \multicolumn{9}{|l|}{2 - Chaisuparasmikul (2006) } \\
\hline \multicolumn{9}{|l|}{3 - Cerezo et al. (2014) } \\
\hline \multicolumn{9}{|l|}{4 - Yeang and Lam (2009) } \\
\hline \multicolumn{9}{|l|}{5 - Kamel and Memari (2019) } \\
\hline \multicolumn{9}{|l|}{6 - Santos et al. (2017) } \\
\hline \multicolumn{9}{|c|}{7 - Kumar and Mahdavi (2001) } \\
\hline \multicolumn{9}{|l|}{8 - Maile et al. (2013) } \\
\hline \multicolumn{9}{|l|}{9 - Noack et al. (2016) } \\
\hline \multicolumn{9}{|l|}{10 - Pratt et al. (2012) } \\
\hline \multicolumn{9}{|l|}{11 - Bazjanac (2008) } \\
\hline \multicolumn{9}{|l|}{12 - Giannakis et al. (2015) } \\
\hline \multicolumn{9}{|l|}{13 - Jeong et al. (2016) } \\
\hline \multicolumn{9}{|l|}{14 - Jones et al. (2013) } \\
\hline \multicolumn{9}{|l|}{15 - Kim et al. (2015) } \\
\hline \multicolumn{9}{|l|}{16 - Lilis et al. (2017) } \\
\hline \multicolumn{9}{|l|}{17 - Welle et al. (2011) } \\
\hline \multicolumn{9}{|l|}{18 - Ramaji et al. (2016) } \\
\hline 19 - Dimitriou et al. (2016) & & & & & & & & \\
\hline
\end{tabular}

Less identifications on Table 18 do not necessarily mean a better or less error-prone process because of how they are rated. For example, number four from Yeang and Lam (2009) achieve a low score because of having many not applicable steps. In addition, number seven from Kumar and Mahdavi (2001) also score low, because of not containing steps to allow for the categorised issues. 


\subsubsection{Stages that Issues Occur In}

After categorising a list of issues and determining what potential issues each reference contains, an understanding of each issue, and how often it can occur is presented. Figure 22 classifies the amount of issues, based on Table 10 and whether a process is performed that could generate an issue. It is counted once per paper of the 19 identified. The most used classes are walls and geometry at 16 each, which surprisingly is not every reference. It is expected that problems could occur with geometry when it comes to testing processes since it is the most used category and as previously identified in section 3.4.1 both IFC and gbXML struggle with aspects of geometry.

The processes that are known to have issues are used on more than half of the references. In addition, important factors such as schedules and loads, and location are not being implemented that much. This does leave room for fewer issues to occur, but the two just mentioned are key to simulations running successfully. The lowest occurring category is duplicate data at three times. This shows that only three references check for duplicate information being produced and then remove it. Shown in Table 15, Maile et al. (2013) have identified this as a known problem that can cause issues during simulation.

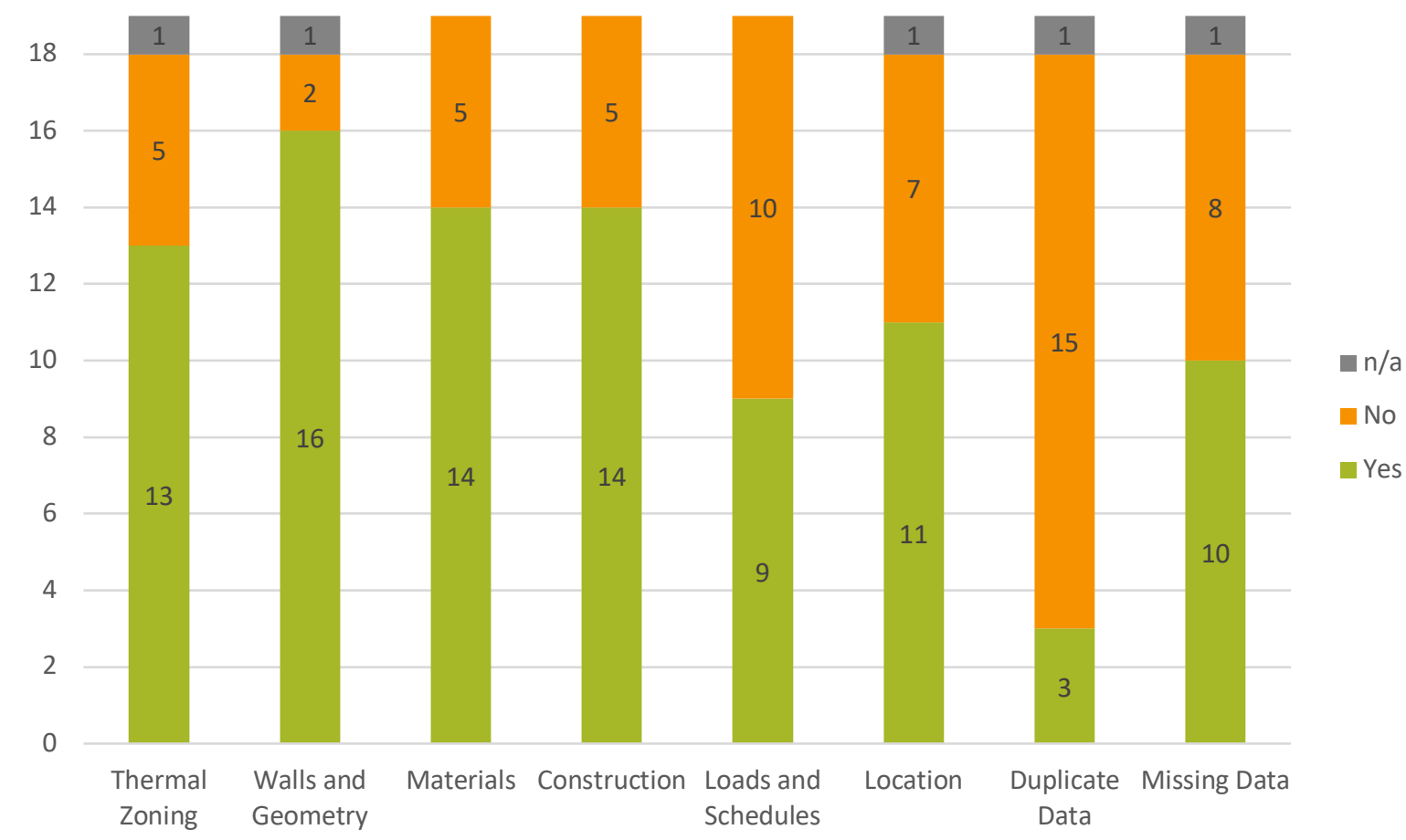

Figure 22: Quantity of Potential Issues per Process

Revit is used the most throughout all the references that specify software use, presented in Figure 23. ArchiCAD, another detailed Architectural modelling software capable of authoring BIM models is not identified. The lesser common Digital Project software is used throughout the categories, 
but only once in each case. Loads and schedules are interesting because they are applied from within a range of software. Location also seems broad but this is due to assumptions of where the weather and location information comes from due to it not being designated in most cases.

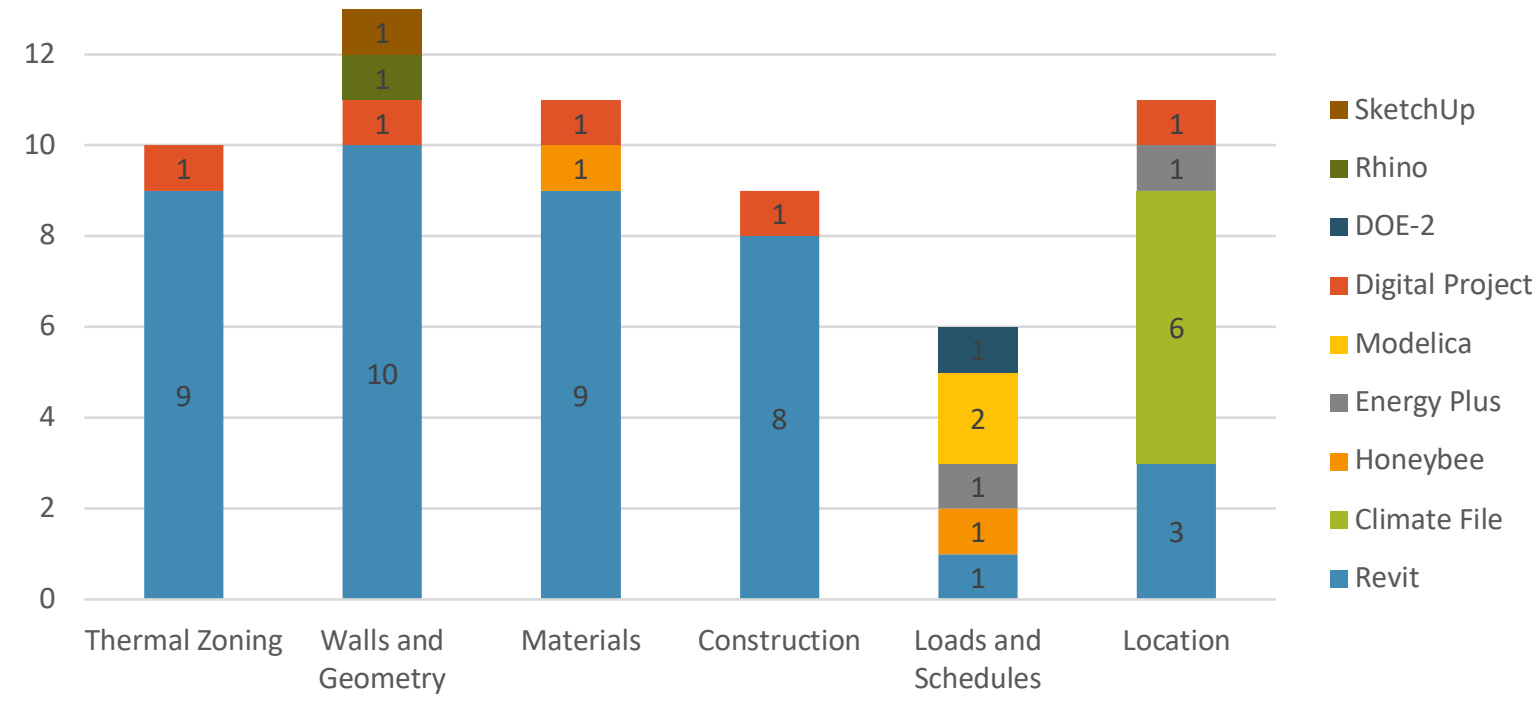

Figure 23: Specific Software or Program Used to Generate Potential Issues

\section{Thermal Zoning}

When an Architect zones a building, it tends to follow geometrical restrictions within a space, while an HVAC Engineer may zone spaces with similar thermal requirements together even if there is geometry separating the conventional spaces. With different approaches to zoning a building or space, which process is best for environmental simulation of lighting, energy, or thermal performance? Moreover, does it matter what one is used for MDH simulations? In this section, the issues, and how thermal zoning is dealt with from the identified references are identified.

Geometrical zoning restrictions that an Architect could apply to design would mean that separate zones are created for each volumetric space. The example in Figure 24 represents each zone as a different colour. With the seven zones, simulations would be more complicated and can take longer periods to simulate. The smaller spaces such as wardrobes would be individually zoned, whereas these should be part of the bedrooms zone. Larger spaces that are open plan or do not have geometry separating them, such as the living room and kitchen, could be combined into one zone even though they have different requirements in terms of load, schedules, etc. These two differences mean there are multiple ways of zoning areas that could produce multiple sets of slightly different results. 


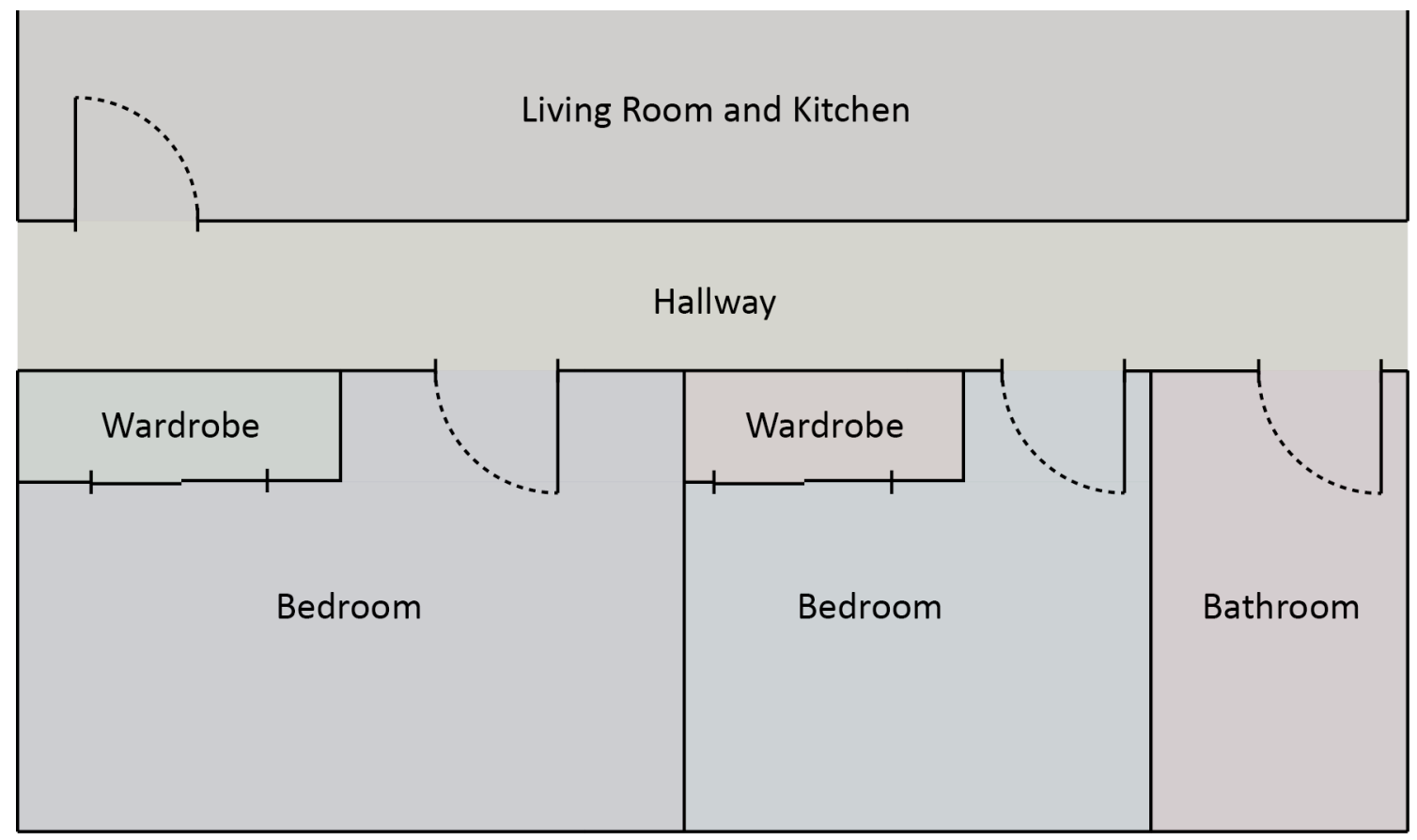

Figure 24: Architectural Geometry Based Zoning

An HVAC Engineer or Simulation Specialist would be far more likely to zone the same house as above in only five zones. In this example, the living room and kitchen are separated due to their differing properties. The wardrobes are absorbed into the bedroom zone, as its size is small and the use would be similar, this thermal zone is extended to cover the adjacent bedroom due to them having identical properties.

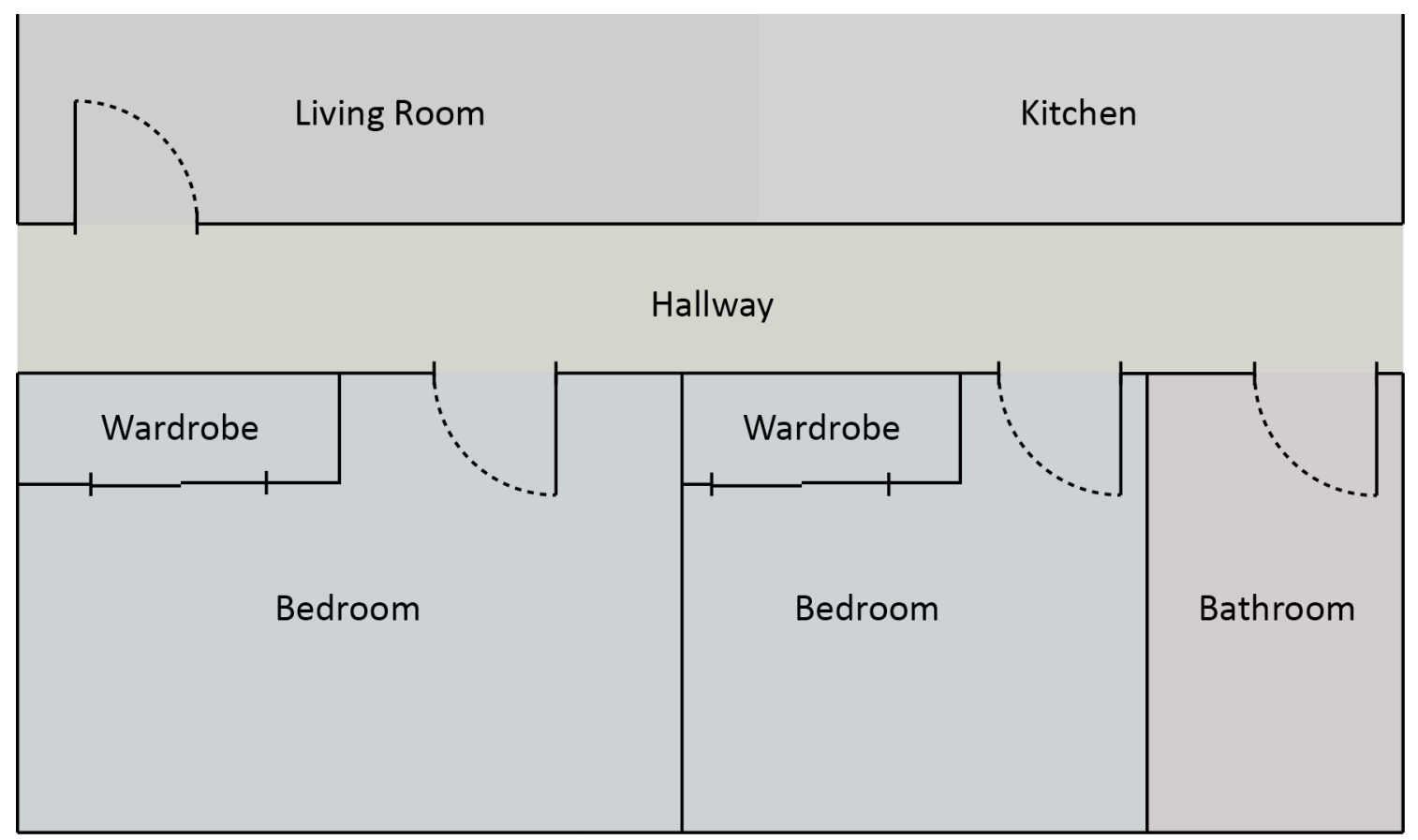


Each of the identified references may consist of a different zoning methodology, approach it in a different way, or not even create thermal zones. The Architectural approach essentially adds complexity to the simulation by adding more zones, while the Engineering approach reduces complexity by only modelling what seems to be necessary. Simulation experts try to reduce the number of thermal zones in a project so that simulation time is also minimalised, some simulations can take hours to days to complete. With computers, increasing efficiencies each year, simulations are becoming faster; therefore, the reasoning to reduce zone numbers to speed up simulations is unfounded. MDH is likely to contain under 15 thermal zones due to its size, meaning even though there are different zoning approaches if zones are being created for reasonable areas it should not affect the outcome of the simulations.

Thermal zoning is identified in 13 processes, while five do not, and one is not applicable. As thermal zoning is necessary for energy simulations, they must be implemented for results to be produced. If a process does not allow for zoning, it will be implemented at the end of the process to ensure that calculations work. Of the processes that allow zones to be produced, nine use Revit, it is by far the most used Architectural modelling software, but it also provides different processes of zoning whether that is space or room. One process uses Digital Project, while the other three processes do not define what specific software is used; just that "CAD" software is involved.

\section{Walls and Geometry}

SketchUp is just one tool among the range of Architectural modelling tools that use twodimensional (2D) planar surfaces within a three-dimensional (3D) space to represent construction geometry. 3D geometry can be created to give the appearance of wall depth visually, although the wall would contain a void making it not solid, displayed in Figure 26. ArchiCAD and Revit are among another set of Architectural modelling software that uses 3D geometry when modelling. The 3D elements are made up of each layer in the wall; this is shown visually and analytically in the software. Figure 26 highlights an example wall from Revit and SketchUp. 

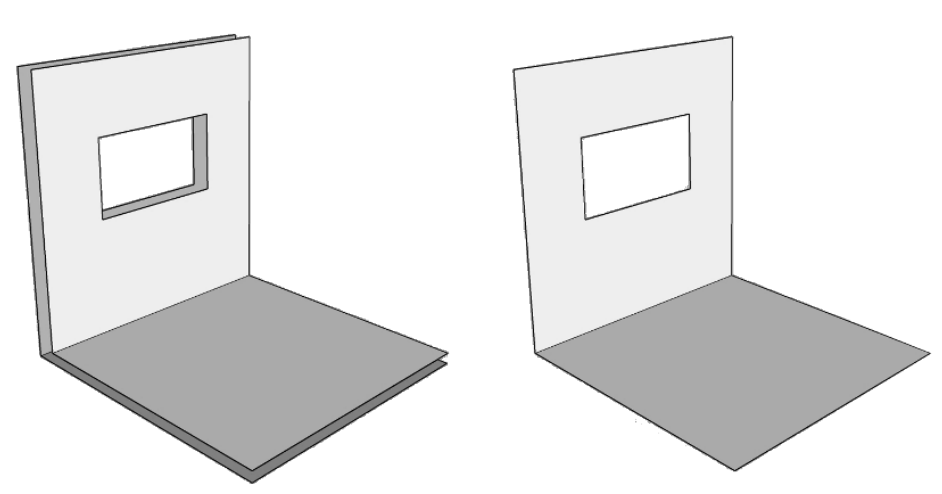

Figure 26: 2D and 3D Geometry Examples

SketchUp - 3D
SketchUp - 2D

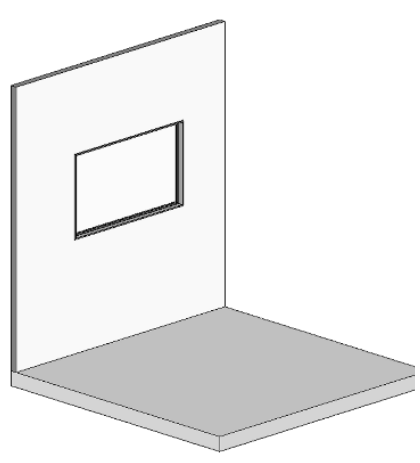

Revit - 3D

Different environmental simulations have slightly different requirements for the walls and geometry. For example, a lighting simulation requires wall thickness or a windowsill so that light can accurately bounce off the surface up into the room and in an energy simulation; visual wall thickness does not matter as each element is just represented as a heat-flow resistance value. If the direct geometry output from SketchUp was to be used in a lighting simulation it would be accurate as wall thickness is not accounted for, however, the 2D version could be used for energy simulation with the addition of R-values. The Revit geometry would have the opposite problems than SketchUp, as wall thickness is physically represented but there are two surfaces to apply an R-value to, the inside and outside which would not work.

In a standard energy simulation, the edges of a zone need to be touching the edges of another zone for them to be classified as internal geometry, if they are not touching any other zone, they can be classified as exposed, which means the geometry is external and is effected by wind and sun. Different calculations are used for internal and external conditions if incorrectly identified simulations can be drastically wrong. Zoning as mentioned in the section above is an important factor for any type of building simulation and the different ways walls and geometry can be modelled effects zoning. Using the SketchUp 3D model, zones would be separated by the thickness of the wall or if automatically zoned a third zone may be placed inside the wall gap. These scenarios create a problem that means simulations either would not run successfully or would be inaccurate. SketchUp's 2D geometry would allow for correct zoning, as no gaps are produced between walls.

ArchiCAD and Revit have different export options allowing different zone definitions to be generated. The edges of a zone or boundaries of a space can be defined into three different 
informational categories whether they be physical (wall, floor, ceiling), or virtual (air wall). These are called first-level, second-level, and third-level space boundaries (Weise et al., 2011).

Due to the role of an Architect or Designer, the most important aspect for a space boundary is to be able to visualise the makeup and finishes of an element correctly on plan drawings, justifying the simplest of conditions, first-level space boundaries (1LSB). They at most contain information to visualise a material, therefore, higher-level analyses cannot be performed. One property that is not seen with the other levels is the ability to have non-planar surfaces (Weise et al., 2011). Second level space boundaries (2LSB) are used within most if not all environmental building performance analysis packages, due to the ability to define boundaries that have differences in material, assembly, or back zone. Materials are represented at a greater level of detail than 1LSB's due to having an R-value, thickness, conductivity, density, and specific heat capacity enabling the use of thermal analysis software. The difference between these two in terms of the surface boundary is shown in Figure 27. Being able to define different assemblies along a space boundary leads to a more representative analysis of wall constructions. A boundary can be defined as either internal or external, if internal, a back zone is applied, and this allows heat transfer between the two adjacent surfaces to take place. Lower level categories cannot perform these tasks (Weise et al., 2011). Third Level Space Boundaries (3LSB) are created when an opaque building element is behind the boundary surface, this usually occurs where a wall intersects another at a right angle. 2LSB's and 3LSB's can be combined to form a completely closed shell around a given space (Weise et al., 2011).

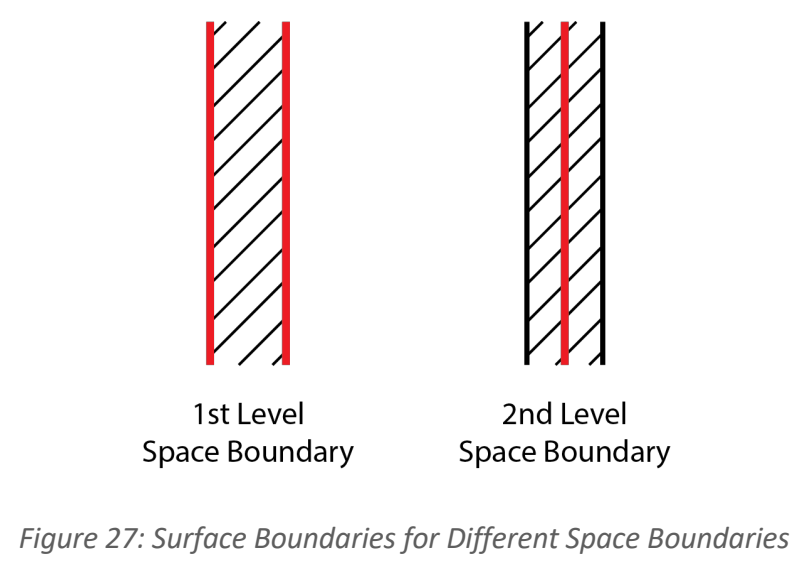

Walls and geometry are identified greatest at 16 times, through the 19 methodologies studied, as set out in Figure 22. Even though the geometry is an important aspect to understand, the two processes that do not discuss any geometry do discuss the general translation of information. Many approaches contain geometrical translation however, as described in section 3.4.1, quite a few issues can be caused by transferring geometry. A majority of wall and geometry information 
is applied from Revit, while a smaller proportion, one approach respectively, uses Digital Project, Rhino, and SketchUp.

\section{Materials}

The distinction between materials is important visually for design and numerically for thermal or lighting simulations. Materials in Architectural models are typically represented by a cut pattern, colour, and surface pattern; these properties are not required for building energy simulation. Due to this, additional thermal and light properties need to be assigned within the Architectural modelling software. A method to bypass the addition of extra properties is to assign an identifiable numerical value to each unique material. Therefore, when material information is exported the corresponding properties to the unique number can be applied in a Middleware. This advanced method is far more complex than simply applying materials in an Architectural model, but it does get around other identified problems with materials. These problems occur when a BIM exporter is used to transform Architectural model data into a transferrable schema. As discussed in Section 3.7.1 and 3.7.2, gbXML and IFC export different amounts of different information. The universal schema IFC can export complete elemental R-values instead of individual material thermal transmittances. If the element ever needs to be broken down or have a material changed, being able to access the original material properties helps, this is possible with gbXML as each layer keeps its value.

Fourteen out of the 19 processes allow materials to be applied. Although this is one of the higher categories, it is surprising that the number is not closer to the total amount of processes because of how vital materials are to a simulation running correctly. Of the 11 processes that specified what software is used, Revit is used nine times, whereas Honeybee and Digital Project are used once each. In these uncommon programs, materials still need to be applied in the same way, and the properties are no different.

\section{Construction}

Constructions are generated from the materials discussed above. Many of the problems occur like missing construction properties for R-value, conductivity, and thermal mass, discussed in section 3.4.1. Therefore, a similar solution to what is mentioned for materials can be used to re-apply or generate construction properties.

Like with materials, constructions are identified in 14 of the processes. Showing that it is used a lot, so there is a higher chance of issues occurring, but that is in direct comparison to constructions being required for many simulations. 


\section{Loads and Schedules}

Zone loads and system schedules are important for simulations as they add the detail that makes models realistic. They are some of the most important sets of information required because simulations cannot run without their implementation. Demonstrated in Figure 22, only nine processes mention how loads and schedules are going to be implemented, the second lowest out of all the categories analysed. As they are required, it must be assumed that other processes also assume they would just be automatically present. The variance of what software provided the schedules and loads, is surprising. Five different processes are used that specify this information, which is more than any other category. Revit, which is highest in most other categories, is only reported once, along with Honeybee, EnergyPlus, and DOE-2, whereas Modelica is used twice. All the different processes used shows that not one approach has proven itself dominant, leaving uncertainty.

\section{Location}

The location of a project matter for many different types of environmental simulations, important information such as temperature, humidity, due point, wind chill, wind direction, wind speed, and solar radiation can be identified and used. All this information is normally provided in the form of a weather file. The type of weather file often used in the simulation is called a typical meteorological year file; this type contains a collection of the average weather over a large period of years. This is opposed to using actual weather recordings for a set period, which is not useful for predicting values such as those produced through simulation.

Weather files can be applied in simulation software and in some Architectural modelling software. If an identified reference does not allow for a weather file during the workflow, results will be irrelevant to any actual location and very generic if the software even allows a simulation to take place.

Figure 22 illustrates that only 11 of the processes describe weather files and a location being used. This information is imported mostly through a climate file as shown in Figure 23, in addition to Revit, EnergyPlus, and Digital Project being used.

\section{Duplicate Data}

Information that is duplicated during the transformation process can cause errors to occur which make simulations crash. Maile et al. (2013) found that most often, geometry is duplicating and being defined in the same location twice. To combat the issue, a model checking process could be implemented which reads the output files and removes information if there are multiple occurrences of it. As gbXML is simpler in its formatting and numbers each element, identifying 
duplicate geometry can be completed systematically through searching each elemental number and determining if it appears twice in succession.

Duplicate data is not identified often, only three times from the processes analysed. This is likely because issues and errors caused by duplicate information are not as common as some other issues.

\section{Missing Data}

Not all information contained in an Architectural model can be translated into other formats. This is the main cause of missing data during translation. Although, there is the potential that information which should be translated is not. Figure 22 demonstrates that ten processes have a strategy to combat missing data. Normally automatic detection and correction like described in Bazjanac (2008), manual detection and correction like in Lilis et al. (2017), or just identification like in Welle et al. (2011). Identifying what information goes missing most often is useful for developing approaches that avoid applying the information too early or that re-apply it at a later stage.

\subsection{Architectural Modelling Software Options}

Four different software's are identified for use in Architectural design. Revit, Rhino, SketchUp, and Digital Project contain the ability to model geometry and apply properties, they do not allow for advanced BPS without additional software. While Revit has energy evaluation software, it is designed for optimising energy only.

Other software not identified through the literature review can also evaluate energy consumption. In ArchiCAD, energy consumption can be estimated; however, it lacks important information such as temperature for comfort. Because only energy is calculated there is little useful information produced for an Architect to design off. These energy values are primarily used for showing a construction meets green rating schemes requirements. In New Zealand, load and schedule values cannot be changed and are locked in default for an office building, essentially making any results for MDH useless.

\subsubsection{Revit Insite and IES VE}

Tools such as Revit Insite or IES VE that are imbedded within existing software are not used as they cannot provide enough quantifiable information to Designers for them to make benifitial design decisions. They do not provide good design anaylsus or sugestions for in a BIM environment, unlike Braasch's example that can consider many situations. 


\subsection{Green Rating Tools}

Each software typically only caters for one environmental or green rating tool. In the case of ArchiCAD and Revit, the focus is towards the Leadership in Energy and Environmental Design (LEED) tool that is prevalent in the United States. Whereas, there is no special focus towards other tools or the ability to choose what one is used. The BIMAccelerationCommittee (2019) of New Zealand says "The use of BIM technologies facilitates more sustainable design techniques" when a "BIM project is evaluated based on NZGBC Green Star, NABERS NZ, or other sustainable criteria". The problem is that these criteria are not implemented into BIM and green rating tools are inherently vaired from the true notion of sustainability.

\subsection{File Export Options}

Most software has dedicated file exporters for IFC, such as ArchiCAD, SketchUp 2020, or Revit. Architectural modelling software Revit can also export information in many different file formats. Two formats standout in literature for having the ability to transfer not only geometric information from Revit to another program but also specific building properties required for BPS, these are gbXML and IFC.

\subsection{1 gbXML Schema}

There are three different export options for a gbXML from Revit. These are spaces, rooms, or energy. Within Revit a small three zoned single-story model is constructed with simple elements, materials, and openings. The model has default loads and schedules which are exported in certain conditions. This model is used to test all exports settings and determine what information can be exported for use within another program.

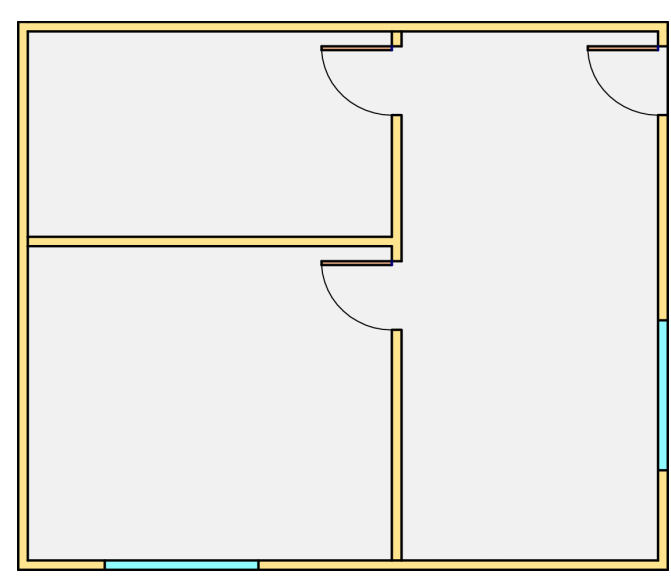

Figure 28: Basic Model Floor Plan 


\section{Space Export}

Spaces are defined in the same way as thermal zones; this means that zones are not just bound by the extents of a room. Exporting a gbXML file using the spaces setting allows for the highest amount of information, which includes loads, schedules, zones, shading, materials, and constructions. These are detailed in Table 19. Loads and schedules are unique to this export option.

Table 19: Information Exported using the gbXML Space Export Option

\begin{tabular}{|l|l|}
\hline Exported & Specific Information \\
\hline Loads & People Number, People Heat Gain, Light Power Per Area, Equip Power Per Area \\
\hline Schedules & Year Schedule, Week Schedule, Day Schedule \\
\hline Zones & $\begin{array}{l}\text { Air Changes Per Hour, Outdoor Airflow Per Area, Outdoor Airflow Per Person, Design } \\
\text { Heat Temp, Design Cool Temp }\end{array}$ \\
\hline Elements & Shades \\
\hline Materials & R-value, Thickness, Conductivity, Density, Specific Heat Capacity \\
\hline Constructions & U-value, Absorptance, Roughness \\
\hline
\end{tabular}

Loads and schedules that can be applied in Revit are successfully exported in detail; both categories are key to a successful thermal or energy simulation. Loads are used to determine how much power is used and the amount of passive heat produced what is added to the spaces. Fractional schedules are used to set occupancy or power densities on an hourly basis, which make up a daily schedule. More depth can be applied in Revit so that variable, week, and yearly schedules can be established. The zone information is exported as one and applied to each space; if a space has different loads or schedules; a new set of zone information is created.

\section{Room Export}

Rooms are defined by the walls throughout a design, meaning an ensuite would be separate from a bedroom, or a cupboard may not be counted as part of a lounge. The rooms export option allows shading devices to export, which does not happen with the energy output. Although there is not as much information as the space export option, different levels of output can be selected. If the complexity is set to simple no shading is exported, this option can be used to get smaller output file sizes or if there are no known shading devices. The complex option exports shading devices in addition to window mullions, although no evidence of this is found in the models. When detailed elements are exported, the materials properties and constructions are produced. If this is not selected no material or constructions would be exported resulting in smaller file sizes, but less key information. 
Table 20: Information Exported using the gbXML Room Export Option

\begin{tabular}{|l|l|}
\hline Exported & Specific Information \\
\hline Elements & Shades \\
\hline Materials & R-value, Thickness, Conductivity, Density, Specific Heat \\
\hline Constructions & U-value, Absorptance, Roughness \\
\hline
\end{tabular}

\section{Energy Export}

Zones for an energy output are set based on spaces; Revit then makes an energy model with analytical surfaces out of the zones. Materials and constructions are exported normally like the other options, although zones are exported individually for each separate zone, unlike the space option. The zone export for energy also contains less information.

Table 21: Information Exported using the gbXML Energy Export Option

\begin{tabular}{|l|l|}
\hline Exported & Specific Information \\
\hline Zones & Outdoor Air Flow Per Person, Design Heat Temp, Design Cool Temp \\
\hline Materials & R-value, Thickness, Conductivity, Density, Specific Heat \\
\hline Constructions & U-value, Absorptance, Roughness \\
\hline
\end{tabular}

This export option fragments elements that have embedded openings (windows or doors) into four or more trapezoid panels as in Figure 29. This would not affect the results from a simulation, but it does greatly increase the complexity of the output gbXML file.

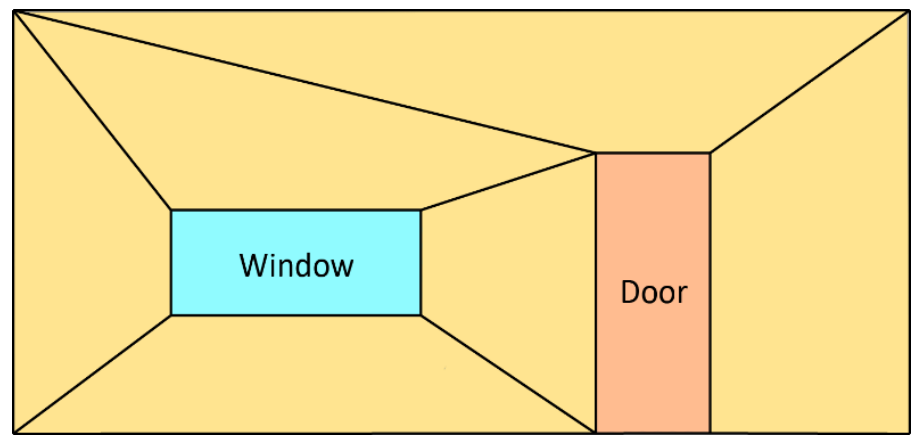

Figure 29: Automatic Geometry Splitting

\section{Construction of gbXML}

Green Building standard XML files are detailed from the bottom-up (Dong et al., 2007). This is shown through how they are constructed. At the lowest and smallest level of detail, materials are defined by a name, R-value, thickness, conductivity, density, and specific heat. When exporting from Revit, this material definition is assigned a material ID in the form of "aimXXXX" where the letters "aim" are a constant and the " $X$ " is given a value. All ID references within a gbXML are defined like this, with the value increasing by one with each new definition made. In a standard 
gbXML produced by other BEM software like OpenStudio, names are already defined and therefore used.

The next level up is the layer ID. It is constructed from layered material ID definitions to form a coherent list of material properties for an element. However, it does not have all the required properties to be labelled a construction. A layer ID looks like a cluster of ID values separated by commas, e.g. aim0001, aim0003, aim0014.

A construction ID is formed from the pre-defined layer ID and additional information. A name, Uvalue, Absorptance, and roughness are also required. The U-value is used in case windows are defined within the construction, as this is the standard for glazing properties. The Absorptance and roughness are required for thermal and light simulations.

Spaces or thermal zones are defined by floor area, volume, and a given definition of space ID, up until this point, no geometry is defined. Under the category of surface ID geometry, elemental type, space ID and construction ID are combined. A surface is defined in space by using Cartesian $\mathrm{X} / \mathrm{Y} / \mathrm{Z}$ geometry, and it is given a construction ID so that it has properties. The terminologies used to generate surface names come from a few areas. Firstly, what direction the outside of the surface is facing is noted, whether it is "T" for Top, "B" for Bottom, "N" for North, "E" for East, "S" for South, or "W" for West. Second, the space ID number for the connecting zone e.g. 1, 2, or 3. If the surface is connected to two, each zone it is interacting with is listed. Next, an "I" is given if the surface is internal or " $E$ " for external. The fourth identifier breaks down what the surface is defined as, either "W" for Wall, "F" for Floor, "R" for Roof, or "S for Shade. Lastly, a unique element number is applied. After this process, a named surface is produced with all the required properties.

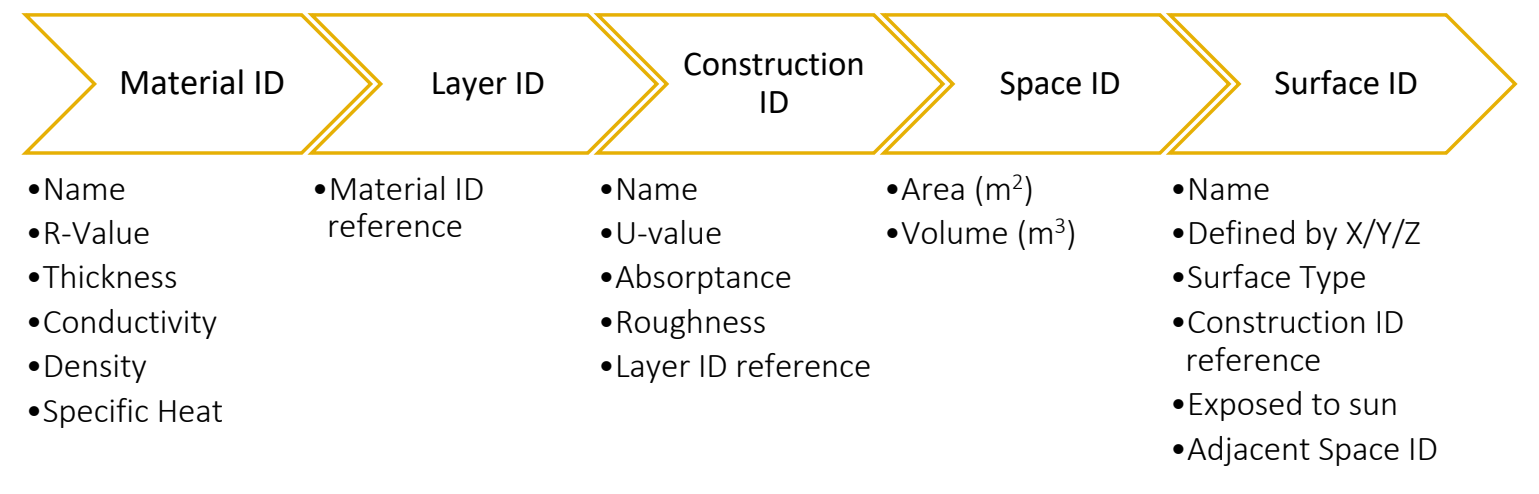




\subsubsection{IFC Schema}

Within the IFC export window, there are many different versions that can be exported, they all have slightly different pre-sets for what is exported or included in the translation. The trial below uses IFC $2 \times 3$ Coordination View 2.0. Specific options must also be selected when exporting with IFC so that desired properties in Table 22 are included.

- Export Revit Property Sets

- Export IFC Common Property sets

- Export Base Quantities

- Export Schedules and Property Sets

Table 22: Information Exported using the IFC Export Option

\begin{tabular}{|l|l|}
\hline Exported & Specific Information \\
\hline Loads & Lighting Load, Power Load, Heat Gain per person, Number of People, \\
\hline Zones & Different loads for each space \\
\hline Materials & Material Names, Thickness \\
\hline Constructions & Roughness, Absorptance, U-value, R-value, Thermal Mass \\
\hline
\end{tabular}

There are additional settings within the export screen that identifies what Level Space Boundaries (LSB) is exported. To determine what information is being exported from Revit, the IFC model is brought back into Revit and examined; the three different options are Zero LSB, $1^{\text {st } L S B}$, and $2^{\text {nd }} L S B$. In all three levels of space boundaries, geometry is visually represented consistently; however, no individual material thermal properties have been transferred through the process. Whole element thermal transmittance is applied to the geometry, so thermal simulations may be possible with these output settings. Zones are only correctly generated to geometrical extents in the Zero and second LSB exports. Zones are generated in the centre of a space but it is not applied across the space for $1^{\text {st } L S B}$. Penetrations in geometry including doors and windows have correctly generated for all options, they have been simplified through the export process as to not include framing, but just a single surface.

\section{Model View Definitions}

Model View Definitions (MVD) is used to define a specific set of information for export to fulfil exchange requirements. They are essentially a trimmed view of the whole project model. A MVD allows for only the required information to be exported e.g. a structural Engineer wants only structural steel elements for a life cycle analysis exported. There are industry defined MVD's, or they can be set by users. 

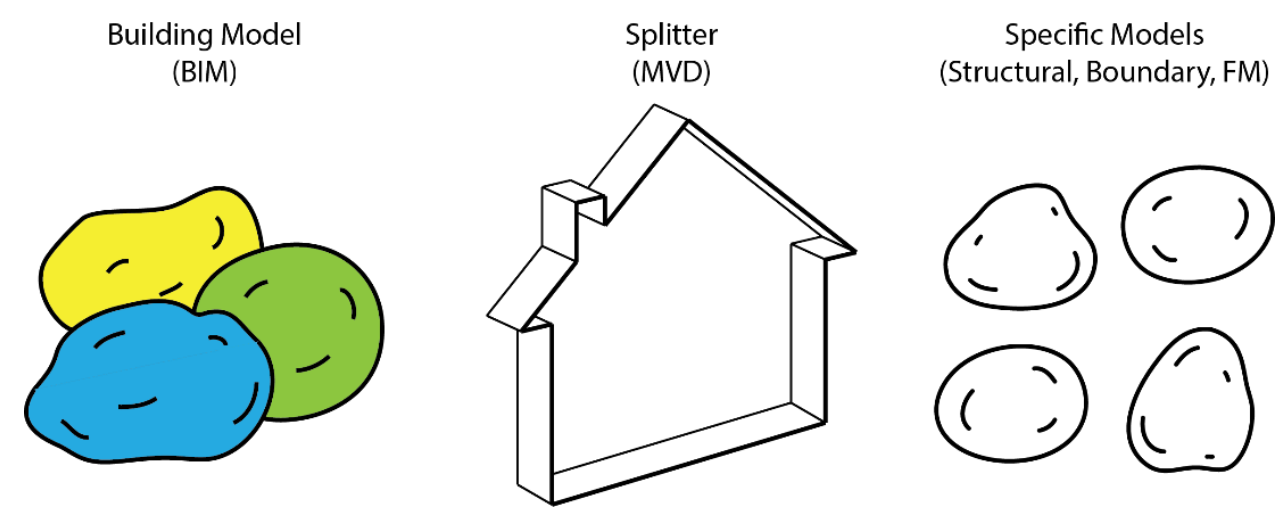

Figure 31: Simplified Example of MVD

The IFC file format schema has specific MVD's for different tasks that could be needed within the AEC industry (BuildingSmart, 2019). Within any program that can export IFC files, there is an option to select what version is required. There are different uses for each; some of the most common are displayed in Table 23.

Table 23: Current IFC Definitions (BuildingSmart, 2019)

\begin{tabular}{|c|c|c|}
\hline Type & Use & Published \\
\hline $\begin{array}{l}\text { IFC2x3 } \\
\text { Coordination } \\
\text { View } 2.0\end{array}$ & $\begin{array}{l}\text { Coordination View is used to allow the sharing of BIM models between } \\
\text { the major disciplines in the AEC industry; it contains definitions of spatial } \\
\text { structure, building, building services elements. }\end{array}$ & 2007 \\
\hline $\begin{array}{l}\text { IFC4 Reference } \\
\text { View }\end{array}$ & $\begin{array}{l}\text { Reference View is used to define a standardised subset of the IFC4 } \\
\text { schema using an MVD. It is suitable for all BIM workflows that are based } \\
\text { on reference models and ID used where the exchange is mainly one } \\
\text { directional. }\end{array}$ & 2015 \\
\hline $\begin{array}{l}\text { IFC4 Design } \\
\text { Transfer View }\end{array}$ & $\begin{array}{l}\text { Design Transfer View is used to provide support for editing of } \\
\text { interconnected elements in BIM, such as inserting, deleting, moving, and } \\
\text { modifying physical building elements and spaces. }\end{array}$ & 2015 \\
\hline
\end{tabular}

Within IFC, a space boundary add-on tool can extend IFC2×3 Coordination View 2.0. The add-ons are presented in Table 24. This add-on allows additional information to be exported that is required for thermal analysis software. The export correctly identifies space boundaries which can be identified as first level or second level, in addition to other requirements such as thermal properties (BuildingSmart, 2019). This add-on and the others available are not used, as they are not mentioned in any processes.

Table 24: IFC Extensions for Specific Tasks (BuildingSmart, 2019)

\begin{tabular}{|l|l|}
\hline Type & Use \\
\hline $\begin{array}{l}\text { Quantity Take-off } \\
\text { add-on View }\end{array}$ & $\begin{array}{l}\text { This allows base qualities for all spatial, building, building services, and structural } \\
\text { elements to be transferred. }\end{array}$ \\
\hline
\end{tabular}




\begin{tabular}{|l|l|}
\hline $\begin{array}{l}\text { Space Boundary } \\
\text { add-on View }\end{array}$ & $\begin{array}{l}\text { This allows BIM to be used in thermal and energy analysis, through adding building } \\
\text { elements to space relationships }\end{array}$ \\
\hline $\begin{array}{l}\text { 2D Annotation } \\
\text { add-on View }\end{array}$ & $\begin{array}{l}\text { This allows for the exchange of additional 2D element representations and } \\
\text { annotations in building models. }\end{array}$ \\
\hline
\end{tabular}

\section{Construction of IFC}

An IFC file is constructed from the top-down, geometry is defined first opposed to the gbXML files previously described (Dong et al., 2007). More information is defined in an IFC file; the same base model uses 9,280 lines of information in the IFC schema, while only 2,365 are used with gbXML schema.

Geometry is constructed from surfaces, which are defined by "IFCPOLYLINE". The polyline is made up of multiple "IFCCARTESIANPOINT" that circle around on itself to close the line.

\begin{tabular}{|c|c|c|c|c|}
\hline Geometry & Material & Material Layer & $\begin{array}{c}\text { Material Layer } \\
\text { Set }\end{array}$ & Property Set \\
\hline $\begin{array}{c}\text {-IFCPOLYLINE = } \\
\text { (PointA\#, PointB\#, } \\
\text { PointC\#, PointD\#, } \\
\text { PointA\#) }\end{array}$ & $\begin{array}{l}\text {-IFCMATERIAL = } \\
\text { (Material Name) }\end{array}$ & $\begin{array}{l}\text {-IFCMATERIALLAYER = } \\
\text { (Material Ref\#, Width) }\end{array}$ & $\begin{array}{c}\text {-IFCMATERIALLAYERSET } \\
=\text { (Layer Ref\#, } \\
\text { Construction Name) }\end{array}$ & $\begin{array}{l}\text {-IFCPROPERTYSET = } \\
\text { (Layer Set Ref\#, } \\
\text { Analytical Property\#) }\end{array}$ \\
\hline
\end{tabular}

Figure 32: Hierarchy of IFC Construction

Materials are defined as "IFCMATERIAL"; they only contain the material name that is applied to them in Architectural modelling software. A large limitation to IFC files is this inability to contain specific material data. Each new line of data in an IFC file is given a unique number, written as \#XXXXX. These numbers are used as an identifier or reference to the information contained on that line.

The material reference numbers are then combined into an "IFCMATERIALLAYER" which takes the unique number and combines it with a material width. This is useful in situations when identical materials are used but at differing thicknesses, as the material is defined once and is just adjusted according to other corresponding width values.

Constructions are generated next using an "IFCMATERIALLAYERSET" definition. This takes the previously defined layer reference number for each layer in the construction and combines this with a construction name. By using this method, complete constructions can be described in a few lines of code.

Property values for constructions are defined one by one. Under the "IFCPROPERTYSINGLEVALUE", heading an array of different information is defined, from 
construction families, constraints, and building phases, which are defined in Revit to roughness, Absorptance, and thermal mass. The values that are defined and useful for building simulation are roughness, Absorptance, heat transfer coefficient, thermal mass, and thermal resistance. A value for each construction set is defined.

Construction properties are applied last by "IFCPROPERTYSET"; this takes the single values described above and combines them into a set that relates to a construction. The references numbers for the required properties are listed along with the relevant "IFCMATERIALLAYERSET". The limitation to this approach is that analytical properties are only known for the overall construction as a whole and values such as roughness or thermal mass must be amassed from a set of differing internal material values.

\subsection{Skills and Abilities of the Intended User}

Weytjens and Verbeeck (2010) determine 47 criteria for energy simulation tools to be Architect friendly. They apply over five sections; data input, output, interface, usability in the development process, and general. Although BPS tools are not being directly rated, these criteria help determine what skill set a typical Architect would have. Table 47 in Appendix One, shows the criteria Weytjens and Verbeeck (2010) identified in one column and the condensed points that an Architect would be expected to be able to do on the other. A typical Architect's skill set for BPS, is reduced to ten points, these are shown below:

1. Select defaults or input small amounts of data quickly

2. Intuitively input or change data to create alternative design options during the early design stage

3. Model a 3D representation of a building

4. Interoperate the results and conformance with building codes via a graphical representation

5. Understand and communicate the impact of design decisions using output information

6. Understand the accuracy of output

7. Use a simple intuitive GUI

8. Use a simplistic tool during the development process to obtain results

9. Learn the intuitive features of a new tool

10. Be able to apply correct materials

Activities that an Architect would be able to undertake when designing in a modelling program such as ArchiCAD or Revit are detailed, but not in terms of information required for simulations. 
Below are activities that are required for thermal and lighting models, which an Architect would not be expected to know:

1. Zoning of spaces for thermal analysis

2. Material properties, such as roughness, thickness, conductivity, density, specific heat capacity, thermal absorbance, solar absorbance, and visible absorbance

3. Loads, such as lighting, plug, people, and occupancy

4. Schedules for temperature, occupancy, computers, and lighting

5. Effects of wall thicknesses

6. HVAC modelling

Architectural modelling and BIM software has purposefully been developed to accommodate the different needs and skills of each discipline so that work can be completed by each expert. Therefore, when determining what workflows literature already offers there must be a consideration in the same way. Some of the processes analysed in Chapter Two have been systematically removed due to them not catering toward the skill set of a typical Architect and being overly complicated. These references by Jeong et al. (2016), Jones et al. (2013), and Kim et al. (2015) contain workflows that would require the user to translate the model into Modelica, another program language specifically for building systems or conduct raytracing to determine what $1^{\text {st }}$ level space boundary walls are connected and create $2^{\text {nd }}$ level spaces boundary's out of them.

There are different ways of approaching thermal and lighting simulation. Complex workflows, take hours for calculations to process can provide highly detailed outputs. Simpler workflows that take comparatively shorter lengths of time and still provide reliant outputs can also be used. The mechanical, electrical, hydraulic, and thermal systems that can be modelled using Modelica puts it on the complex side, it is useful in areas where a higher level of detail can be of benefit over what would be obtained in conventional software. As systems, this detailed are rarely planned during early concept or development design stages there is no need to have Architects follow a complex workflow.

\subsection{Medium Density Housing Models}

A large amount of new MDH is going to be constructed in New Zealand due to the KiwiBuild scheme. Enabling Architects to simulate and predict how their designs affect the occupants and the environment through BPS is invaluable. 
Translations process should be able to work on a model with a reasonable degree of complexity. A single-family home would be too simple to confirm that the process should work with an Architect's model. This makes MDH a logical complexity, with the advantage that if it works for $\mathrm{MDH}$ it can be expected for the simpler forms of normal single-family homes it will also be successful and for the much larger commercial buildings. Starting with MDH allows larger commercial buildings to be tested in the future. Although larger buildings are bigger, they may not be as complex.

\subsubsection{Model Complexity}

Two different model complexities are used during the testing of approaches. Attia et al. (2009), finds that the accuracy of a tool, combined with its ability to simulate complex buildings makes BPS more Architecturally friendly.

In this thesis, the model complexities are used to understand how different factors such as those from Weytjens and Verbeeck (2010) are translated. Different issues may occur because of the model complexity and information that can be stored in that model.

An intervention of BPS within the early design stages mean more decisions can be made. This can enhance Architect's confidence in design, but also allow developers to accept them more easily (Xie and Gou, 2017). Architects identify what factors would enhance the design the most by way of understanding the effects on energy and climate during the development process (Weytjens and Verbeeck, 2010). These factors are:

- Glazing percentage impact on Energy, identified by $80 \%$

- Insulation impact on Energy, identified by $76 \%$

- Orientation impact on Energy, identified by $50 \%$

- Thermal mass impact on Energy, identified by $50 \%$

- Glazing percentage impact on Overheating, identified by $63 \%$

- Shading devices impact on Overheating, identified by $50 \%$

Most of the references identified to translate building information are tested with low complexity models, like single-story houses with less than five thermal zones. Although there are eight higher than low complexity model used, highlighted in Table 25.

Table 25: Identified Model Complexities

\begin{tabular}{|l|c|c|c|}
\hline \multicolumn{1}{|c|}{ Reference } & Model Complexity & Zones & Stories \\
\hline 1 - Kota et al. (2014) & Low & 1 & 1 \\
\hline 3 - Cerezo et al. (2014) & Low & 1 & 1 \\
\hline 4 - Yeang and Lam (2009) & Low & 3 & 1 \\
\hline
\end{tabular}




\begin{tabular}{|l|c|c|c|}
5 - Kamel and Memari (2019) & Low & 4 & 1 \\
\hline 6 - Santos et al. (2017) & Low & 1 & 1 \\
\hline 13 - Jeong et al. (2016) & Low & 1 & 1 \\
\hline 18 - Ramaji et al. (2016) & Low & 2 & 1 \\
\hline 2 - Chaisuparasmikul (2006) & Medium & $10+$ & 1 \\
\hline 10 - Pratt et al. (2012) & Medium & 3 & 2 \\
\hline 15 - Kim et al. (2015) & Medium & 3 & 2 \\
\hline 12 - Giannakis et al. (2015) & High & $10+$ & 2 \\
\hline 16 - Lilis et al. (2017) & High & $10+$ & 2 \\
\hline 19 - Dimitriou et al. (2016) & High & $10+$ & $20+$ \\
\hline 14 - Jones et al. (2013) & Very High & 273 & 3 \\
\hline 17 - Welle et al. (2011) & Very High & 21 & - \\
\hline 7 - Kumar and Mahdavi (2001) & Not Defined & - & - \\
\hline 8 - Maile et al. (2013) & Not Defined & - & - \\
\hline 9 - Noack et al. (2016) & Not Defined & - & - \\
\hline 11 - Bazjanac (2008) & Not Defined & - & \\
\hline
\end{tabular}

\section{Low Complexity Model}

The low complexity model is used to test the most basic aspects of a translation process; materials, zones, and geometry, although more detailed information is stored within the model. Figure 33 presents the geometry of the three-zone MDH model, the specific properties that are tested with this model are:

- External corner connections

- Internal to external adjacent connections

- Internal adjacent connections

- Material properties transformation

- How windows act

- How doors act 


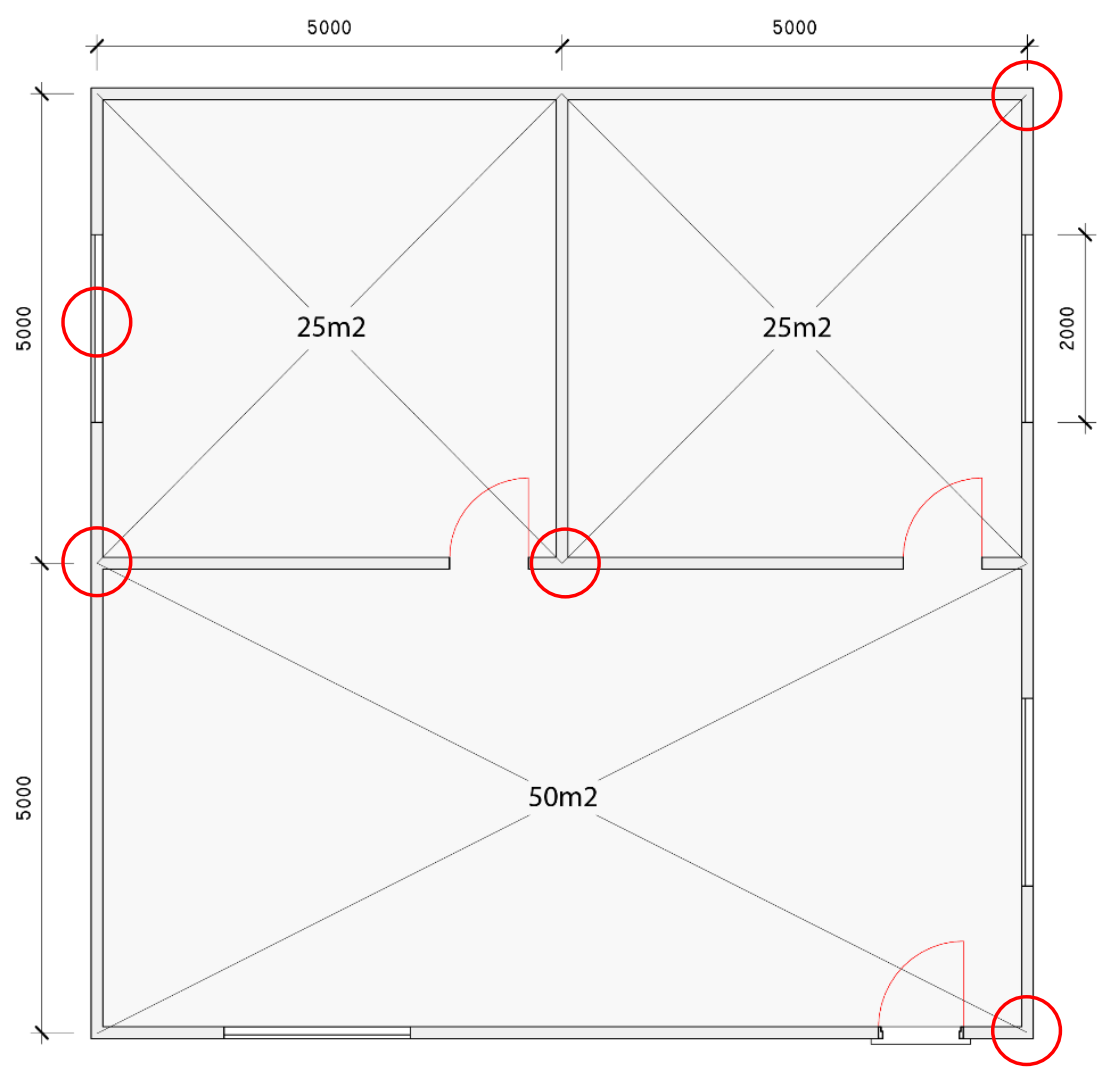

Figure 33: Floor Plan of Low Complexity Model

The model has a floor area of $100 \mathrm{~m}^{2}$ and includes four external windows, one external door, and two internal doors. Internal windows are not modelled as it is expected they would be treated in the same manner as doors due to them both being sub surfaces of a wall. In terms of energy modelling, there is minimal reasoning to model internal doors, however, as they are modelled in a standard Architectural model, it is important to know what reaction could occur during building translation.

Higher-level detail such as furniture, fittings, and even door handles are assumed not to be modelled. This is because they wouldn't have any impact on simulation results and that there would be a way to export the Architect's model with these complexities removed.

\section{High Complexity Model}

The complex MDH model that is produced by Ganda (2019) was converted to contain zones and identical material properties to the low complexity model. The model's floor area is approximately $132.2 \mathrm{~m}^{2}$. Testing the identified processes with this more detailed geometry has the possibility to highlight errors that a simple model could not. The model contains 14 unique zones laid out in Figure 34; there is a mix of internal and external elements, including air walls. Air walls are the technical term used for the virtual divide between open plan spaces, in energy modelling they 
must be defined as a "wall" that has zero resistance to heat transfer. The model also includes geometry representing cupboards, stairs, and voids making it a closer representation to a real Architectural model.
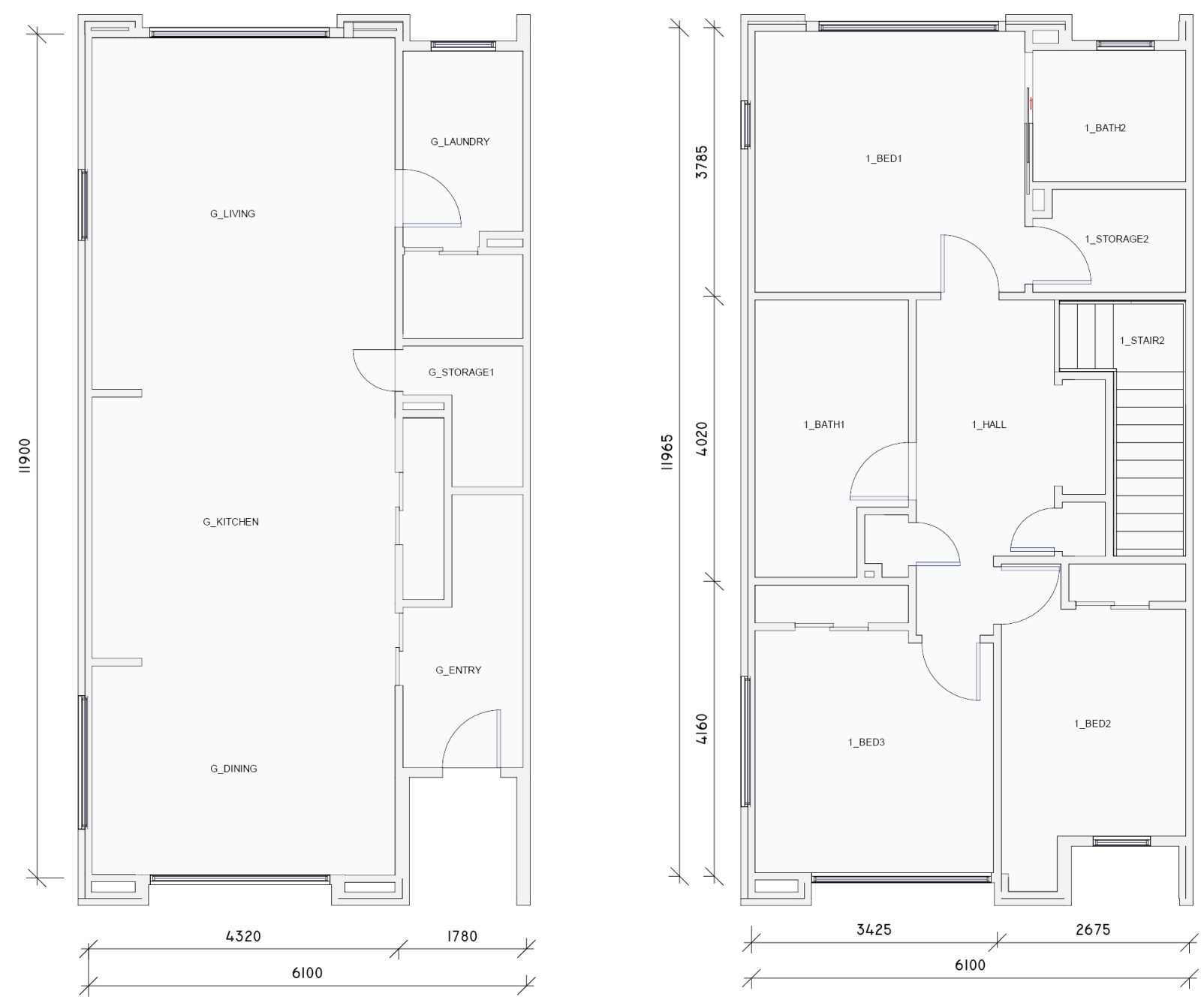

Figure 34: Floor plan of High Complexity Model

When applying spaces to a complex model the limits or extents must be correctly defined to intersect with bounding geometry, otherwise, air elements are generated. The only difference between this model and the low complexity one is the number of zones, surfaces, and the fact that it is two stories.

\subsubsection{Elemental Constructions}

Constructions are designed for Wellington, New Zealand, which is in Climate Zone two and requires the R-values as set out in Table 48 of Appendix One. 
The overall elemental R-values for each makeup shown in the tables below are calculated using the iso-thermal planes method determined in NZS4214 (2006). This is used to account for the effects of thermal bridging, which occur when two or more materials are enclosed within one plane of a wall. In all the basic makeups below, timber and insulation are combined into one layer.

Materials that cover $100 \%$ of the wall plane can be simply added together. Surface thermal resistance also needs to be applied; this is the resistance of air touching the internal and external surfaces of an element. NZS4214 (2006) defines the internal coefficient as $0.09 \mathrm{~m}^{2 \circ} \mathrm{C} / \mathrm{W}$ and the external coefficient as $0.03 \mathrm{~m}^{2 \circ} \mathrm{C} / \mathrm{W}$. These coefficients are accounted for when calculating the overall R-value; however, they are not inputted in Revit as they are automatically applied in energy simulation software during the latter stages. All R-values are either directly from NZS4214 (2006) or calculated using the conductivity values provided in the same standard.

A basic external wall makeup is shown below with corresponding component R-values; the timber framing percentage is assumed $20 \%$, therefore $80 \%$ insulation. This set of elements produce an overall R-value of 1.9 , which is the code minimum for Wellington, New Zealand.

Table 26: External Wall

\begin{tabular}{|l|l|c|c|c|}
\hline Element & Type & Layer & Percentage & R-value $\left(\mathrm{m}^{2{ }^{\circ}} \mathrm{C} / \mathrm{W}\right)$ \\
\hline Cladding & $\begin{array}{l}\text { Bevel back Weatherboard, } \\
18 \mathrm{~mm}\end{array}$ & 1 & $100 \%$ & 0.14 \\
\hline Air Barrier & Building Paper & 2 & $100 \%$ & 0.01 \\
\hline Timber Framing & Pine, 90mm & 3 & $20 \%$ & 0.75 \\
\hline Insulation & Fiberglass R-2.2 & 3 & $80 \%$ & 2.20 \\
\hline Wall Lining & Gypsum Plasterboard, 10mm & 4 & $100 \%$ & 0.05 \\
\hline
\end{tabular}

Internal walls do not have a resistance requirement due to them being inside the thermal envelope; however, they still require a construction. Below is a simple internal wall that also has the internal coefficient above applied twice. Additionally, the timber percentage is set at $20 \%$.

Table 27: Internal Wall

\begin{tabular}{|l|l|c|c|c|}
\hline Element & Type & Layer & Percentage & R-value $\left(\mathrm{m}^{2 \circ} \mathrm{C} / \mathrm{W}\right)$ \\
\hline Wall Lining & Gypsum Plasterboard, $10 \mathrm{~mm}$ & 1 & $100 \%$ & 0.05 \\
\hline Timber & Pine, $90 \mathrm{~mm}$ & 2 & $20 \%$ & 0.15 \\
\hline Wall Lining & Gypsum Plasterboard, $10 \mathrm{~mm}$ & 3 & $100 \%$ & 0.05 \\
\hline
\end{tabular}

\begin{tabular}{|l|l|}
\hline Overall R-value & 0.42 \\
\hline
\end{tabular}


The external roof is assumed as a basic timber framed roof that is directly fixed or battened with a flat ceiling. The frame used is $7.5 \%$ timber and made up from $90 \mathrm{~mm}$ rafters at $600 \mathrm{~mm}$ centres.

Table 28: External Roof

\begin{tabular}{|c|c|c|c|c|}
\hline Element & Type & Layer & Percentage & R-value $\left(\mathrm{m}^{2 \circ} \mathrm{C} / \mathrm{W}\right)$ \\
\hline Roofing & $\begin{array}{l}\text { Corrugated Steel, Building } \\
\text { Paper }\end{array}$ & 1 & $100 \%$ & 0.01 \\
\hline Air Barrier & Roof Space & 2 & $100 \%$ & 0.11 \\
\hline Timber Framing & Pine, $90 \mathrm{~mm}$ & 3 & $7.5 \%$ & 0.75 \\
\hline Insulation & Fiberglass, R-3.2 & 3 & $92.5 \%$ & 3.20 \\
\hline Wall Lining & Gypsum Plasterboard, 10mm & 4 & $100 \%$ & 0.05 \\
\hline & & & Overall R-value & 2.90 \\
\hline
\end{tabular}

The external suspended floor frame consisting of $140 \mathrm{~mm}$ joists at $600 \mathrm{~mm}$ centres provides a timber percentage of $7.5 \%$.

Table 29: External Floor

\begin{tabular}{|l|l|c|c|c|}
\hline Element & Type & Layer & Percentage & R-value $\left(\mathrm{m}^{2 \circ} \mathrm{C} / \mathrm{W}\right)$ \\
\hline Timber Framing & Pine, 140mm & 1 & $7.5 \%$ & 1.17 \\
\hline Insulation & Fiberglass, R-1.4 & 2 & $92.5 \%$ & 1.40 \\
\hline Flooring & Plywood, $14 \mathrm{~mm}$ & 3 & $100 \%$ & 0.10 \\
\hline
\end{tabular}

\begin{tabular}{|l|l|}
\hline Overall R-value & 1.60 \\
\hline
\end{tabular}

Although there are no set R-value requirements for internal elements, they still require constructions. The internal ceiling and floor have the same construction set but in reverse order from one another, set out in Table 30.

Table 30: Internal Ceiling/Floor

\begin{tabular}{|l|l|c|c|c|}
\hline Element & Type & Layer & Percentage & R-value $\left(\mathrm{m}^{2 \circ} \mathrm{C} / \mathrm{W}\right)$ \\
\hline Flooring & Plywood, $14 \mathrm{~mm}$ & 1 & $100 \%$ & 0.10 \\
\hline Timber Framing & Pine, $90 \mathrm{~mm}$ & 2 & $20 \%$ & 0.15 \\
\hline Ceiling Lining & Gypsum Plasterboard, 10mm & 3 & $100 \%$ & 0.05 \\
\hline
\end{tabular}

\begin{tabular}{|l|l|}
\hline Overall R-value & 0.47 \\
\hline
\end{tabular}

These constructions are applied to the low complexity and high complexity Architectural models.

Windows are represented with a single value, which accounts for the frame and glazing combination. For a large single glazed window, this can be assumed as a U-value of 6.7018. 


\subsubsection{Applying Materials to Architectural models}

Each element has different insulation levels, timber thickness, or timber percentage, this result in individual thermally bridged core values. These are tabled below.

Table 31: Core R-values for Each Element

\begin{tabular}{|l|c|c|}
\hline Element & Thermal Conductivity & R-value \\
\hline External Wall & 0.05673 & 1.58 \\
\hline Internal Wall & 0.60000 & 0.15 \\
\hline External Roof/Ceiling & 0.03300 & 2.73 \\
\hline External Floor & 0.10150 & 1.38 \\
\hline
\end{tabular}

Constructing each material in Revit can be done at any Architectural development stage before export for BEM. Some of the default materials in Revit have thermal properties applied, but they are inconsistent with values given in NZS4214 (2006), meaning everything needs to be set up for New Zealand climate standards. Once an example of each material is applied in Revit, they can be drawn from in the future.

\subsubsection{Quality Assurance}

The Household Energy End-use Project (HEEP) conducted by BRANZ identifies an average dwelling in Wellington consumes $10,860 \mathrm{kWh}$ per year with a standard error of 790kWh, or 3,620 kWh per occupant with a standard error of 280kWh (HEEP, 2010). These values along with some simple quality assurance checks are used on each model simulation to ensure that reliable results are being produced. The process also allows for easy identification of simulations that do not run as expected.

As two different models are used, different energy consumptions are generated. The low complexity model is not based off a real dwelling; therefore, the expected total kWh energy consumption is lower than what HEEP estimates. For the high complexity model based off a real MDH model the overall energy consumption should be within the range defined by HEEP (2010).

Once simulation results are determined to be representative according to HEEP, an additional simulation is conducted where insulation levels are halved. For a climate like Wellington, New Zealand, the heating loads would be expected to increase and cooling loads decrease. The test is done to ensure that the simulation engine is behaving correctly and as expected. If unexpected results occur then the initial base test may not be able to be trusted either. 


\subsubsection{Thermal Bridging}

Thermal bridging is a complicated issue in energy simulation as constructions are made by layering materials on top of each other in planes as discussed previously. The problem when using hand calculations is simple to solve through using the iso-thermal method and formula. However, in energy simulation software OpenStudio, or Architectural modelling software Revit there is no way to input multiple materials in one plane. Moreover, until building material technology that allows for no thermal bridging are commonplace these problems will occur. There are a few ways to approach the thermal bridging problem, each with their own limitations and assumptions.

The first method is to create two different constructions for an element; they would both have identical layers that cover $100 \%$ of the wall. One of the constructions has only insulation applied in the core and another with only timber in the core. If the wall has a $20 \%$ timber percentage, that proportion of the wall area is applied with the timber only construction, while the rest has the insulation construction applied. In Figure 35, a simple wall diagram in elevation shows how this would work.

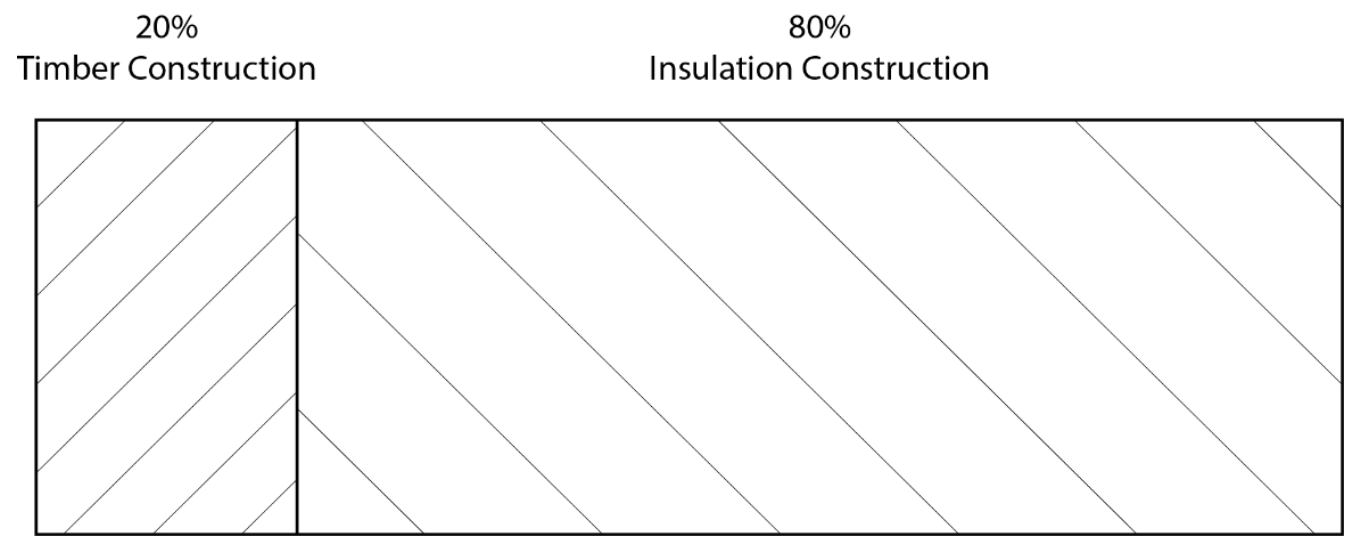

Figure 35: Two Wall Constructions Applied to One Wall

This approach would not work in Architectural design due to the miscommunications it would cause on plan or detail drawings. If there were a process to alter the walls after export from Architectural modelling software to make them like this, the approach would work for energy or heat loss simulations. This approach would also make lighting simulation possible as it simply requires geometry, but moisture simulations would be drastically incorrect due to making the assumptions that this approach requires.

The second approach involves calculating an overall R-value for the core material using the isothermal planes method and applying it to $100 \%$ of the wall. Below are two images of a simple wall that are made of cladding, building paper, timber, insulation, and gypsum plasterboard. However, if the Architectural model simply incorporates both structure and insulation then it produces the 
wall on the left that is double the thickness as insulation and timber are each represented. The wall on the right has the correct thickness, but requires an unreal combined resistance comprising $20 \%$ timber and $80 \%$ insulation, which makes moisture risk and transfer difficult to model.
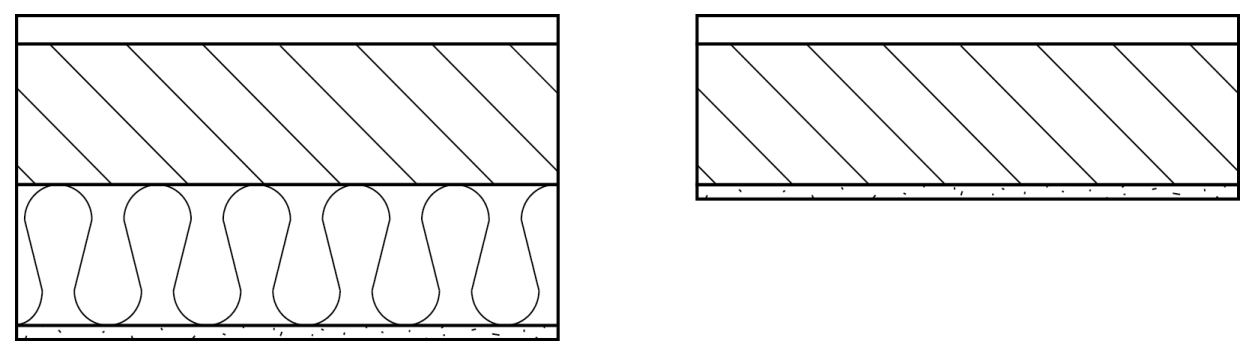

Figure 36: Layered Wall in Architectural Model (Left) and Condensed Wall (Right)

Due to the increased thickness, the larger wall has a high R-value. To keep the thickness and resistance the same as defined above, the calculated thermally bridged R-value is applied to the core material. This approach assumes that there is even distribution of resistance and other material properties such as density, specific heat capacity, and emissivity. This method allows for correct energy, heat loss, and lighting simulations, but not moisture like the first approach. When drawn in an Architectural modelling program it looks correct due to the makeup of material layers.

The third and most complicated approach involves meticulously modelling the location of each timber element with the correct material as shown in Figure 37.In theory; this method would provide correct energy, heat loss, lighting, and moisture simulations. Nevertheless, manually creating this type of geometry is not practical. If an automated program could generate framing construction this method would become viable for use in simulations.

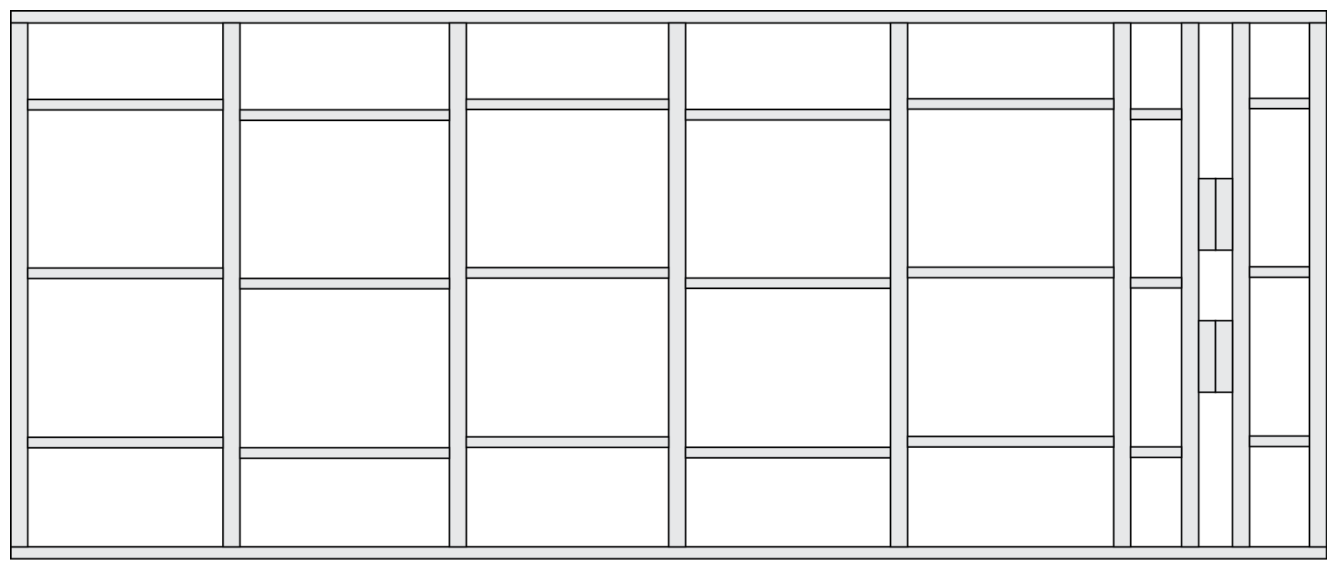

Figure 37: Individually Framed Wall Geometry

Out of the three approaches above, the second is used because of the representation in Architectural software along with the energy and lighting simulation uses. Although, the model 
produced through the transformation process would not be able to be used for moisture simulations due to the construction limitations.

\subsection{Ranking Translation Processes}

To identify what transfer process can provide the most beneficial information relating to how Architects can use BPS; the references highlighted in section 2.6.1 are ranked. Ranking enables papers with the most valuable information to be identified from the set of relevant literature. After applying the measures of success in section 3.2.1 to the 19 identified transfer processes, a list of ranked references is produced, shown in Table 32.

Table 32: Rankings of Identified References

\begin{tabular}{|c|c|}
\hline Ranking & Transfer Process Identified In \\
\hline $1^{\text {st }}$ & - Noack et al. (2016) \\
\hline $2^{\text {nd }}$ & - Ramaji et al. (2016) \\
\hline $3^{\text {rd }}$ & - Santos et al. (2017) \\
\hline $4^{\text {th }}$ & $\begin{array}{l}\text { - Giannakis et al. (2015) } \\
\text { - Dimitriou et al. (2016) }\end{array}$ \\
\hline $5^{\text {th }}$ & - \\
\hline $6^{\text {th }}+$ & $\begin{array}{c}\text { - Pratt et al. (2012) } \\
\text { - Bazjanac (2008) } \\
\text { - Welle et al. (2011) } \\
\text { - Kamel and Memari (2019) }\end{array}$ \\
\hline
\end{tabular}

Some references achieve the same rankings as each other, causing there to be two fourth places in the top five. Because of this, the top five can clearly be identified. There is also a clear distinction to where the lower scoring references placed.

The graph displayed in Figure 38 provides the overall point rating and a breakdown for each of the identified references. Only nine are identified as possible to test based on the criteria set out in section 3.2.2. The excluded references are shown in the graph below with a saturated hue. Some of these excluded references score highly, like Lilis et al. (2017), which would have been in $4^{\text {th }}$ equal, had testing been possible. As Figure 38 highlights, not every reference scores in each category. The successful ones detail the process, identify what software information is transferred to, and have minimal steps where information could be lost. 


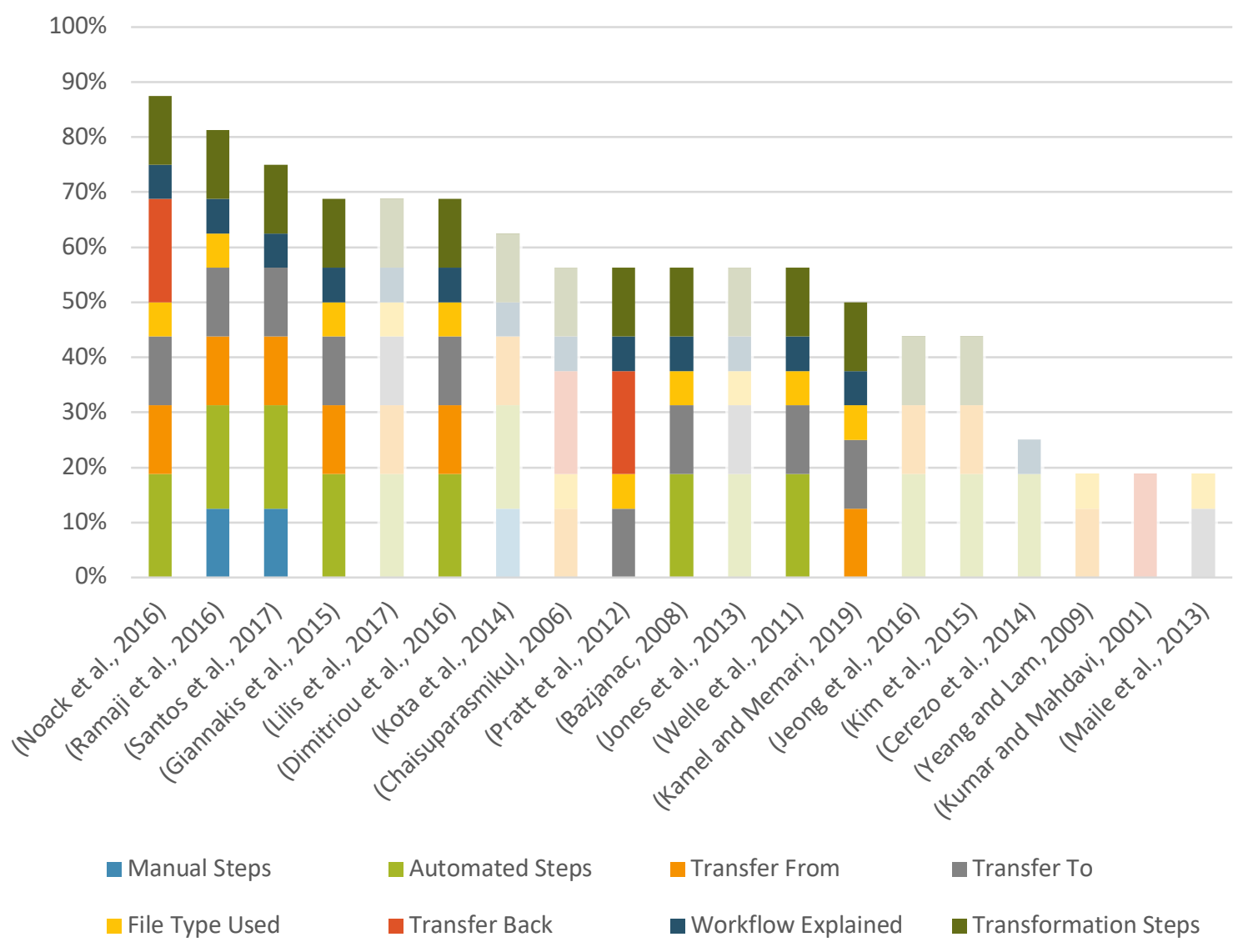

Figure 38: Ranked Translation Processes

The set of 19 references are not all used in testing because:

- They are not all able to be replicated due to lack of information, incomplete process, or unavailable software

- There is no need to test everything when there is a finite number of potential categories of processes

- Similar processes identify the same issues and successes

Five are used in testing as the processes within them cover a range of different areas, including file schema, design tool, simulation tool, middleware, or additional processes. The additional four unused references that placed in $6^{\text {th }}$ place would not provide any new information due to them not ranking highly.

These processes cover four different categories that are given in the next chapter. Each category contains at least one process that is representative of all types in that category because of the schema, Middleware, and simulation program. The number that was used is sufficient to test the range of types of process. 
Chapter Three: Methodology 
Chapter Three: Methodology 


\section{Chapter Four: Testing}

This chapter reports testing of Architectural model to thermal model translation processes with the goal of answering the questions proposed in chapter one, 'What must be improved for BIM to be used in building performance simulation?' and 'Can BIM be used by an Architect to calculate performance during the early stages of design?'.

In this chapter, the processes that have been identified based on the measures of success set, are tested through following the processes and methodology described in chapter three. These processes of translation are completed in as much detail as possible so that issues and errors can be identified. The energy consumptions of each approach are compared to Quality Assure that unknown errors are not making it through the testing stage. 


\subsection{Testing Translation Processes}

Seven translation processes are identified from the top five rated references. The approaches take somewhat similar steps to achieve translation as seen from a high-level overview of all processes in Figure 39. The most common nodes within the process are shown to be Revit, IFC or gbXML, an algorithm, IDF, and EnergyPlus. Although only approach " $C$ " from Noack et al. (2016) takes this exact path, most of the other processes vary slightly from it. The most differing approach is in Santos et al. (2017), which uses modelling tool Rhino and doesn't require direct manual translation due to the Middleware Grasshopper.

Identified processes can seem similar from a high-level view, but they contain more detailed or comprehensive steps between stages. An example of this is the difference between approaches " $A$ " and "B" from Noack et al. (2016). While they both use Revit as the modelling tool and the gbXML schema in the translation step, " $A$ " describes a weather file being implemented, and " $\mathrm{B}$ " contains an additional file check and edits to remove degenerate information.

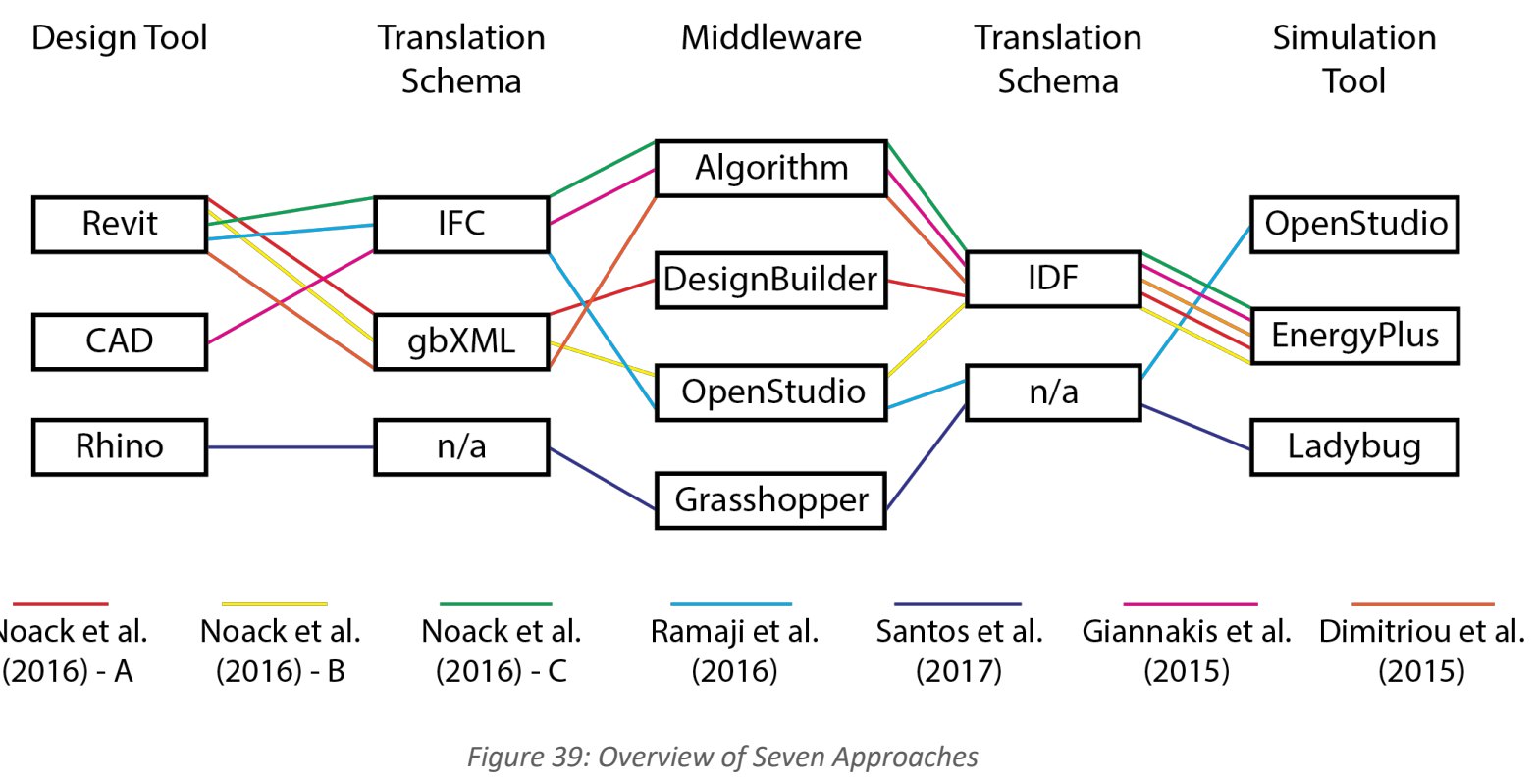

An overview of the three approaches proposed by Noack et al. (2016) is set out in Figure 39, while a more detailed workflow for each approach is in Figure 41, Figure 44, and Figure 51. There are slight differences in the processes between approaches " $\mathrm{A}$ " and " $\mathrm{B}$ ", being the translator tool, weather data, and the location information from a component database is implemented. The component database described in this reference is not used in any tested workflows due to access and already having quality assured material properties. In the three processes, the design4energy (D4E) repository is also ignored, due to it solely acting an online repository to transfer results to the BIM authoring tool. These approaches are all from the identified reference paper by Noack et 
al. (2016), which is ranked first with a score of $87.5 \%$. It achieves the requirements in all sections apart from manual steps. As described in section 2.9, the positive aspects of Noack et al's processes are that there are different approaches depending on what file type is exported from the Architectural modelling software. Noack et al. (2016) uses specialised validator and conversion tools are used in some cases, these are not publicly available, and so any steps that these are required in are skipped or completed manually. This is a limitation of accessing processes from academic literature.

Second is an approach from Ramaji et al. (2016) that scores $81.25 \%$ in the measures of success assessment. This approach uses IFC as the translation schema and software OpenStudio for simulation. The original process is unique when compared to the others in Figure 39. A detailed image of this is shown in Figure 50.

The process ranked in third with 75\%, is from Santos et al. (2017), while it doesn't involve any file transformations or translations, energy results are shown to be produced and even visualised on the original model. The process of using software Rhino and Grasshopper is presented in Figure 47.

With two approaches ranked in fourth place, Giannakis et al. (2015) and Dimitriou et al. (2016) each achieved a score of $68.75 \%$. Although they achieved the same ranking though the same measures, the fundamental design tool and translation schema are different. A more detailed process is set out in Figure 53 and Figure 46.

As some approaches cannot be tested directly from the literature source, adjustments are made for them to have the highest chances to be successful. The process for how each process is tested is in Figure 40. DesignBuilder is not used, as access is not available during the thesis period, due to it merely being used for file translation in a "black box", OpenStudio is used in its place. Discussed previously, three of the processes use a publicly unavailable algorithm or set of code to translate IFC and gbXML BIM files, into an IDF format. Since processes cannot be tested without knowing what the algorithm or code does, a known process in OpenStudio is used to perform the Middleware translation. 


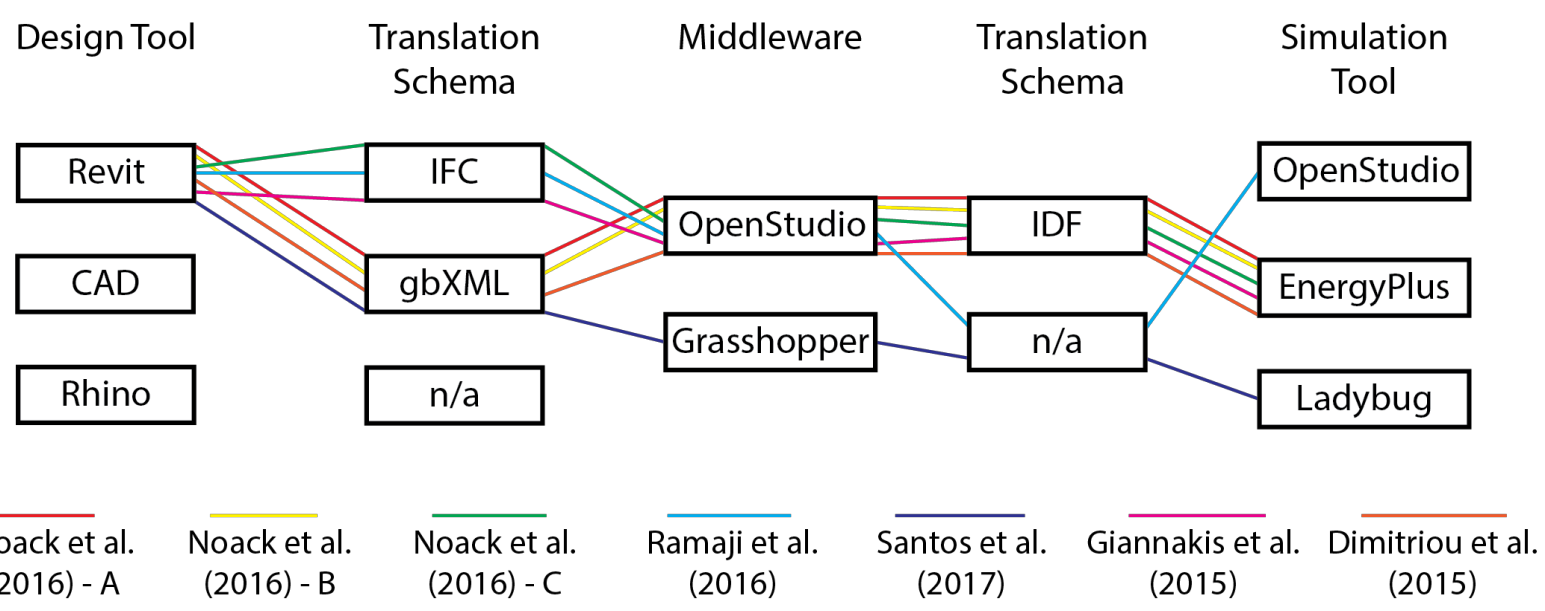

Figure 40: Overview of Adjusted Approaches

Six of the seven tested translation processes use OpenStudio. This means that problems or issues relating directly to OpenStudio are identified more often, however, issues relating to all other sections of the process except for approaches by Santos et al. (2017) and Giannakis et al. (2015), remain the same. Santos et al. (2017) uses Grasshopper as a VPL but does not generate results off an Architectural model produced in common Architectural software. This workflow is altered so that a gbXML from Revit is imported and tested as it has already been shown that the back end of the approach works. The process from Giannakis et al. (2015) is only modified slightly to include Revit, due to no Architectural modelling software being specified in the original.

Based on Figure 40 and presented in Table 33, the seven processes can be sorted into four groups by how similar their individual stages are. The processes are grouped firstly by translation schema, then Middleware, and lastly simulation program. The first grouping contains three approaches making it the most common. The first process is the most simplistic and could even be simplified once more; however, none of the references found suggested that approach.

Table 33: Categorised Processes

\begin{tabular}{|c|c|l|}
\hline Category & Generic Process (Translation $\rightarrow$ Middleware $\rightarrow$ Simulation) & \multicolumn{1}{c|}{ Approach From } \\
\hline 1 & gbXML $\rightarrow$ OpenStudio $\rightarrow$ EnergyPlus & $\begin{array}{l}\text { Noack et al. (2016) - “ } A \text { ” } \\
\text { Noack et al. (2016) - “ } B \text { ” } \\
\text { Dimitriou et al. (2016) }\end{array}$ \\
\hline 2 & gbXML $\rightarrow$ Grasshopper $\rightarrow$ Honeybee & Santos et al. (2017) \\
\hline 3 & IFC $\rightarrow$ OpenStudio $\rightarrow$ OpenStudio & Ramaji et al. (2016) \\
\hline 4 & IFC $\rightarrow$ OpenStudio $\rightarrow$ EnergyPlus & $\begin{array}{l}\text { Noack et al. (2016) - “C" } \\
\text { Giannakis et al. (2015) }\end{array}$ \\
\hline
\end{tabular}




\subsubsection{Category One: gbXML $\rightarrow$ OpenStudio $\rightarrow$ EnergyPlus \\ Process "A" in Noack et al. (2016)}

Approach " $\mathrm{A}$ " contains adjustments to the processes for: manual replication of proprietary code or processes, and the Middleware translation tool used.

Table 34: Summary of Approach "A" from Noack et al. (2016)

\begin{tabular}{|c|c|}
\hline Low Complexity & High Complexity \\
\hline Adjusted Process Failed & Adjusted Process Failed \\
\hline Manually Edited Process Successful & Manually Edited Process Successful \\
\hline
\end{tabular}

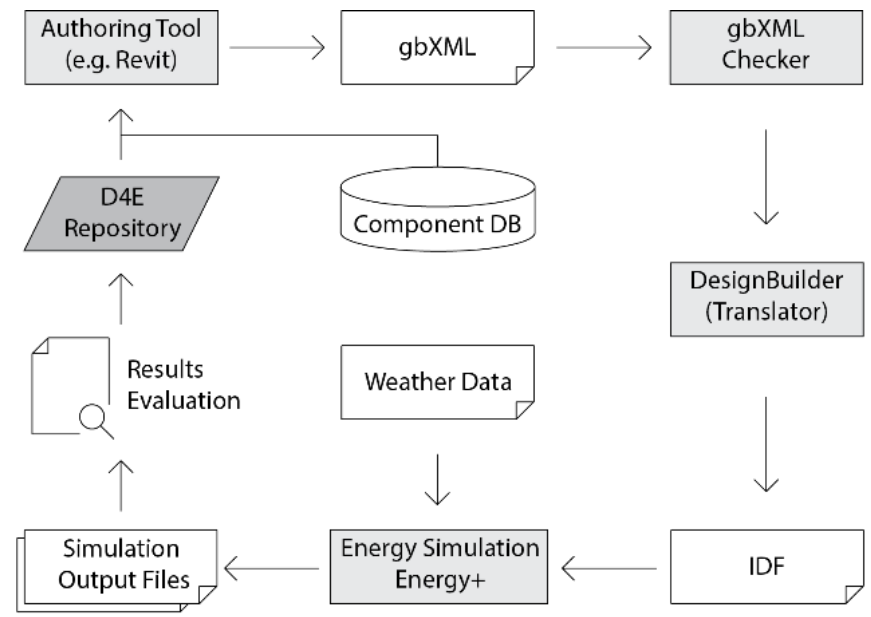

Figure 41: Approach "A" from Noack et al. (2016)

The approach in Figure 41 uses their privately developed gbXML checker and software DesignBuilder. These steps were swapped out for a manual gbXML check and the energy simulation software OpenStudio as the checker software was unavailable. This process includes a manual gbXML check that ensures materials, properties, constructions, and heating and cooling information is available. As discussed in Noack et al. (2016), any missing information is added where appropriate. DesignBuilder is used as a "black box" in the process to translate building information from a gbXML file format to an IDF file format; therefore, due to the simple nature of its use, a similar software can be used in its place. The software OpenStudio also contains the necessary properties to import a gbXML and export an IDF for simulation in EnergyPlus. The low complexity model using the adjusted approach fails at the simulation stage due to a partial translation of information from the Middleware OpenStudio. 


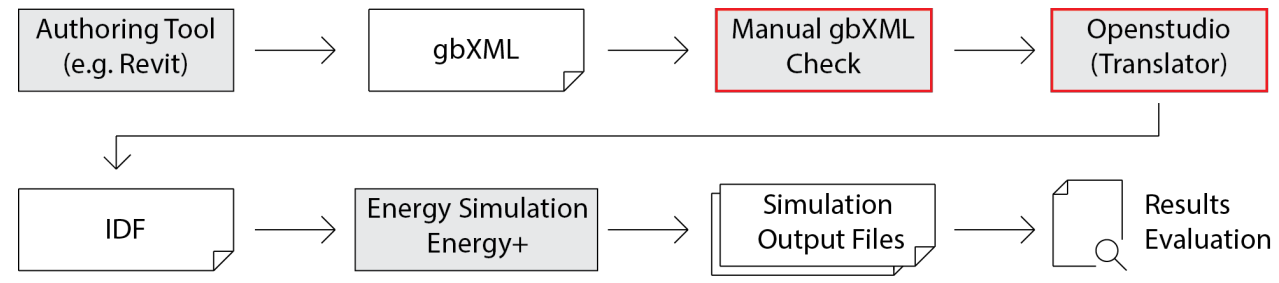

Figure 42: Adjusted Approach "A" from Noack et al. (2016)

With the high complexity model following Figure 42 the gbXML file is exported correctly. During the manual check, process it is found that ceilings are exported with two identical space ID's. This common problem would cause the elements not to be generated due to duplicate adjacent geometry in the text. A snapshot of what the exported code looks like can be viewed in Figure 61 in Appendix Two. The duplicate section of code is removed. Then the corrected file is imported into OpenStudio and an error appears, set out in Figure 62 of Appendix Two. This error is found again to be in relation to duplicate code, however for "Air" surfaces between spaces. Removing these occurrences solves the issue. Then the model was viewed in OpenStudio and a blatant error was exposed. In the Architectural model a ceiling space had been modelled between floors and was not associated as part of any zone. Therefore, a gap is translated through the export process; Figure 43 shows how the model is represented within OpenStudio. This can be corrected by either not modelling the ceiling space within Revit, increasing the zone height to intersect the bottom of the first story floor, or lowering the upper zones to intersect with the ceiling in OpenStudio. As the model would currently fail under any simulation methodology, due to surfaces being incorrectly matched this error would occur in every high complexity simulation proposed. Therefore, the original model is edited to remove the internal ceiling space between floors. This space was implemented for another purpose; it does not represent how an Architect would model multiple stories. However, it does provide an example of how models act if spaces are incorrectly modelled. Although the Architectural model is fixed for future simulations, the model fails translation into the Middleware software.

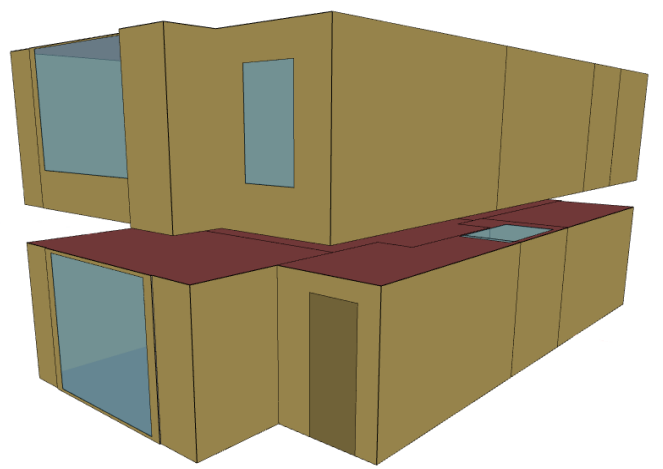


Process "B" in Noack et al. (2016)

Approach " $\mathrm{B}$ " contains adjustments to the processes for: manual replication of proprietary code or processes, and translation tool used instead of proprietary translation process.

Table 35: Summary of Approach "B" from Noack et al. (2016)

\begin{tabular}{|c|c|}
\hline Low Complexity & High Complexity \\
\hline Adjusted Process Failed & Adjusted Process Failed \\
\hline Manually Edited Process Successful & Manually Edited Process Successful \\
\hline
\end{tabular}

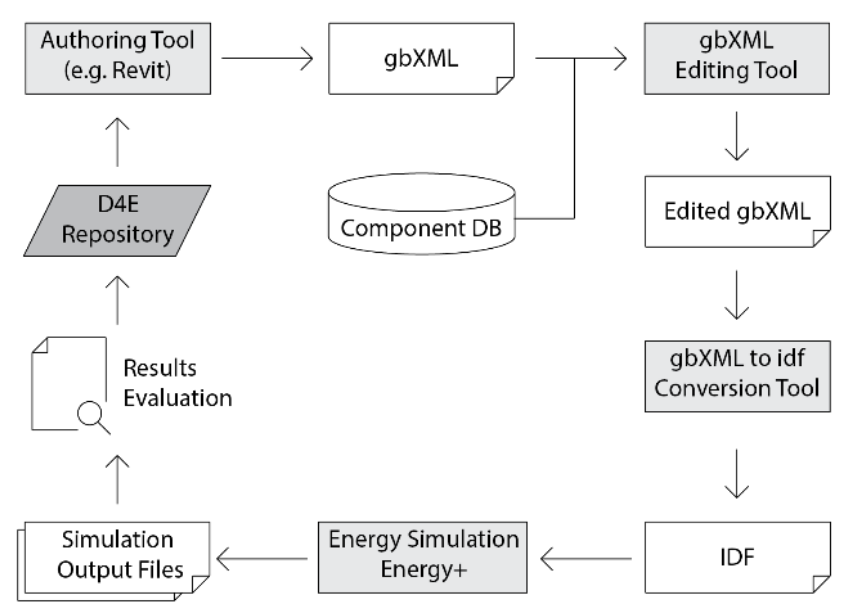

Figure 44: Approach "B" from Noack et al. (2016)

Approach "B" from Noack et al. (2016) visualised in Figure 44 uses additional tools in the process. The tools are also not publicly available; they are described as a gbXML editing tool and a standalone gbXML to IDF conversion tool. The gbXML editing tool is used to implement changes to building characteristics, such as material properties, and to add additional information that may be required. The proprietary gbXML to IDF tool is used so that all valid information is converted; making sure that nothing is missed or skipped from the automated processes. Any additional information that may be required can also be added at this stage. The two processes described above are implemented differently in the testing approaches, and an additional manual IDF check is completed. Following the process in Figure 44 with the low complexity model, exports from Revit with no errors, therefore, no information needs to be removed or added to the gbXML File. Opening the gbXML file in OpenStudio reveals correct geometry and materials, but once again, the schedules and loads have been removed. A manual check of the IDF shows that through the model translation, correct schedules and loads are not applied. The model runs using EnergyPlus, but no results are produced.

When the adjusted approach in Figure 45 was used information could be manually added and checked for. This resulted in a successful energy simulation. 


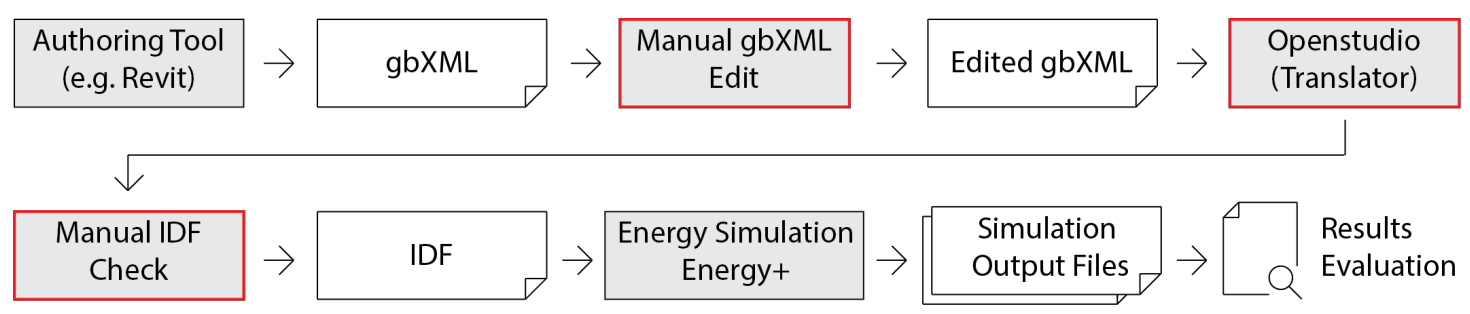

Figure 45: Adjusted Approach "B" from Noack et al. (2016)

The approach provides benefits when the high complexity model was used, as editing the gbXML export from Revit is part of the process. This step could be automated with a simple executable code to remove duplicate information; however, a manual edit is performed instead. Allowing the issues caused in approach " $\mathrm{A}$ " not to occur. Importing the gbXML into OpenStudio so that an IDF can be exported is successful, but the translation is only partial due to missing information in OpenStudio.

Manually editing both the low and high complexity models allows for successful simulation processes.

\section{Process in Dimitriou et al. (2016)}

The approach from Dimitriou et al. (2016) contains adjustments for the Middleware translation tool used.

Table 36: Summary of Approach from Dimitriou et al. (2016)

\begin{tabular}{|c|c|}
\hline Low Complexity & High Complexity \\
\hline Adjusted Process Failed & Adjusted Process Failed \\
\hline Manually Edited Process Successful & Manually Edited Process Successful \\
\hline
\end{tabular}

The last approach in this category is from Dimitriou et al. (2016), it also follows the most common point in each section of Figure 40. The original process in Figure 46 uses an algorithm and set of mapping instructions to translate information from gbXML to IDF. This is replaced with OpenStudio as the translation tool because the mapping instructions are not described in enough detail for testing to take place.

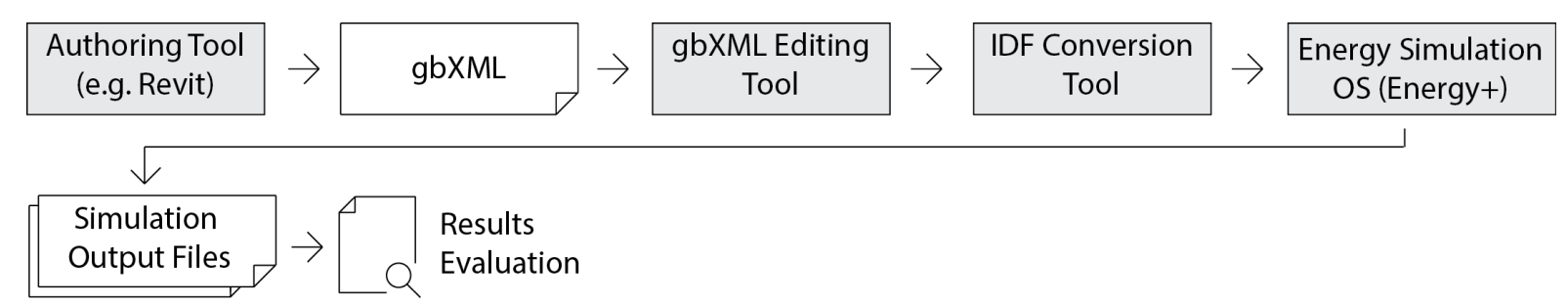

Figure 46: Approach from Dimitriou et al. (2016) 


\subsubsection{Category Two: gbXML $\rightarrow$ Grasshopper $\rightarrow$ Honeybee}

\section{Process in Santos et al. (2017)}

The approach from Santos et al. (2017) contains adjustments for: type of model used, and removal of surface flattening process.

Table 37: Summary of Approach from Santos et al. (2017)

\begin{tabular}{|c|c|}
\hline Low Complexity & High Complexity \\
\hline Adjusted Process Failed & Adjusted Process Failed \\
\hline Manually Edited Process Successful & Manually Edited Process Failed \\
\hline
\end{tabular}

\begin{tabular}{|c|c|c|c|c|c|c|c|c|}
\hline $\begin{array}{l}\text { CAD Modelling } \\
\text { Tool (Rhino) }\end{array}$ & $\rightarrow$ & $\begin{array}{l}\text { Planarization } \\
\text { Algorithm }\end{array}$ & $\rightarrow$ & $\begin{array}{c}\text { Planar Model in } \\
\text { Rhino }\end{array}$ & $\rightarrow$ & $\begin{array}{l}\text { Link Model to } \\
\text { Grasshopper }\end{array}$ & $\rightarrow$ & $\begin{array}{c}\text { Energy Simulation } \\
\text { Energy+ }\end{array}$ \\
\hline
\end{tabular}

\section{Figure 47: Approach from Santos et al. (2017)}

The approach aims to calculate energy consumptions for spaces constructed in the modelling software Rhino using the process in Figure 47. However, it does have an advantage over Revit or ArchiCAD, the ability to draw complex curved shapes. Through the process, complex models can even be used in energy simulations. Because of following the approach, a double-curved geometrical space is created within Rhino; the complex curves on this space cannot be simulated in EnergyPlus without alteration. The model is brought into Grasshopper and attempts are made with the mentioned approaches in Santos et al. (2017) using Kangaroo 2 and an algorithm to translate the geometry to flat quadrants. However, due to the complexities and the lack of a clear workflow description within Grasshopper, the process of flattening geometry could not be completed. A planar model is used to test the other steps of the workflow as if the flattening algorithm had worked. Linking the model into Grasshopper for assessment is a simple process within the VPL interface, while applying the necessary components for a simulation using EnergyPlus is slightly more complicated. No issues are produced from these two steps. The VPL workflow used runs EnergyPlus using the geometry information input, produces results, and visualises them on the model.

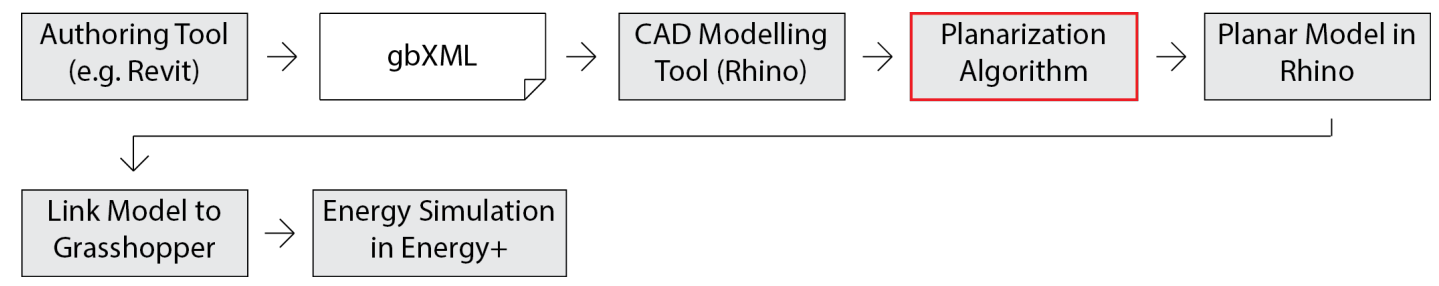

Figure 48: Adjusted Approach from Santos et al. (2017) 
The process works with a planar model; therefore, the low complexity model was tested. As integrating BIM models into the workflow is not discussed in Santos et al. (2017) two more steps are added to the process in Figure 47 to create the process above. Additionally, the flattening step is ignored due to the complexities described above, and that the model is already planar. Using the Grasshopper plugin Honeybee, gbXML files are imported into the VPL. The component used to do this is called "Honeybee_gbXML to Honeybee", it claims to import geometry, constructions, and boundary conditions into the modelling environment. However, it does not automatically translate and apply loads, schedules, or HVAC systems. This component is applied to the start of the process created for the approach above, which imports the model into Honeybee representative geometry and zones. Visualisation of the model shows the zones, geometry, windows, and doors which are imported through this component. With geometry imported, another component to check that materials have successfully been applied is used, this visualises the applied material constructions over the model geometry. The model is successfully linked to Grasshopper that allows for simulation to take place using an EnergyPlus component. However, the simulation fails.

When a complex model was used, the approach was not successful. The approach produces results; however, they are incorrect due to issues identified by the energy simulation program. Visualised in Figure 49, most zones are consuming a reasonable amount of energy per $\mathrm{m}^{2}$, while one zone in particular, is consuming up to five times as much.

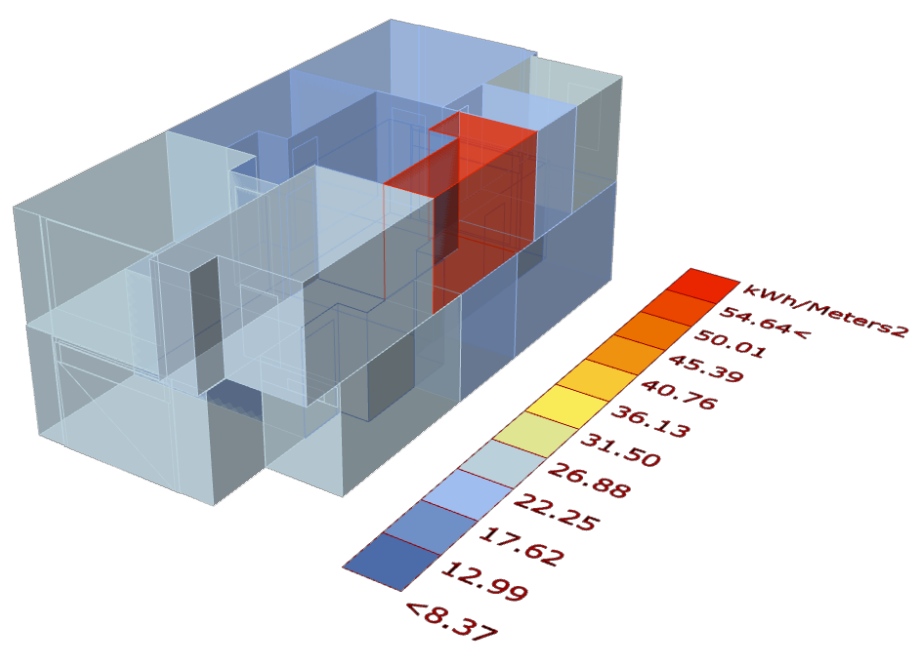

Figure 49: Energy Results Displayed Over the Complex Model

The low complexity and high complexity models both failed during simulation. However, the simulation was successful when manual editing was used. 


\subsubsection{Category Three: IFC $\rightarrow$ OpenStudio $\rightarrow$ OpenStudio}

Process in Ramaji et al. (2016)

The approach from Ramaji et al. (2016) contains no adjustments.

Table 38: Summary of Approach from Ramaji et al. (2016)

\begin{tabular}{|c|c|}
\hline Low Complexity & High Complexity \\
\hline Adjusted Process Failed & Adjusted Process Failed \\
\hline Manually Edited Process Successful & Manually Edited Process Successful \\
\hline
\end{tabular}

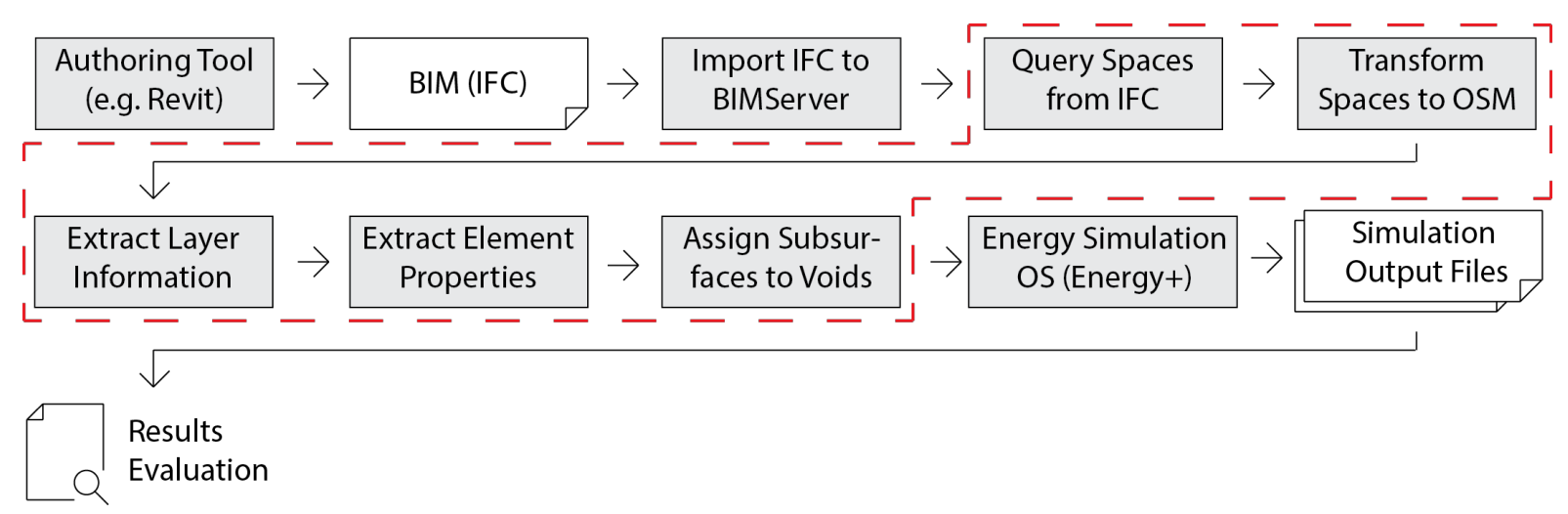

Figure 50: Approach from Ramaji et al. (2016)

The only category to simulate in the same program as its Middleware is from Ramaji et al. (2016). It contains five steps that consist of producing a BIM model in the IFC schema format, importing the model into BIMserver, running the BIMserver analysis and translation, simulating energy consumption, and producing results. Communicated in Figure 50, the highlighted steps are automatically run within the BIMserver software. This approach works slightly differently to the other processes tested in Figure 40, as it simulates energy consumption from the Middleware and does not export information a second time. This approach uses BIMserver, which is open-source software that allows for the storage of IFC based BIM models (BIMserver, 2014). It is used in this, along with an OSM serializer to translate IFC files to the OSM within OpenStudio. A serializer is a mapping process that lays out what equivalent information is defined as in another program. No steps in this process have to be altered from the original proposed.

The Low complexity model exports from Revit successfully, however it was missing a lot of critical information when imported to OpenStudio. This ultimately causes a failure in the simulation step.

The high complexity model is edited initially to change the ceiling of the top floor to be a roof after discovering that IFC cannot contain external ceiling geometry. The IFC output contains the same types of information as the low complexity model, just a lot more of it. The increased complexity 
and size can still be handled by BIMserver and the transformation only takes a little longer. As uncovered with the previous model there is information that does not transfer properly or does not even exist in the OSM file. Due to only a partial translation of information, the simulation fails. Manually editing the process allows both model complexities to successfully simulate.

\subsubsection{Category Four: IFC $\rightarrow$ OpenStudio $\rightarrow$ EnergyPlus}

Process "C" in Noack et al. (2016)

The approach from Noack et al. (2016) contains no adjustments.

Table 39: Summary of Approach "C" from Noack et al. (2016)

\begin{tabular}{|c|c|}
\hline Low Complexity & High Complexity \\
\hline Adjusted Process Failed & Adjusted Process Failed \\
\hline Manually Edited Process Failed & Manually Edited Process Failed \\
\hline
\end{tabular}

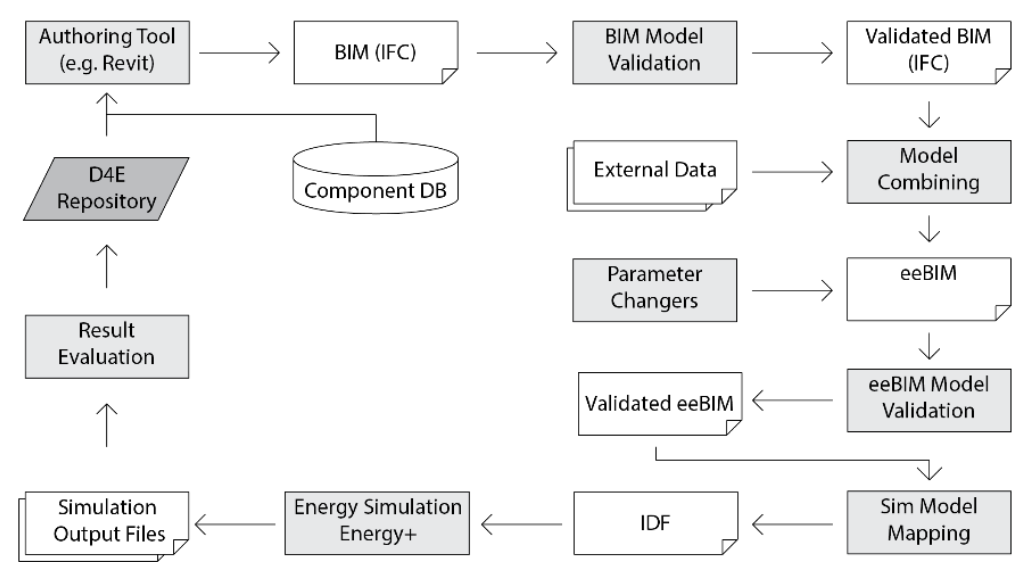

Figure 51: Approach "C" from Noack et al. (2016) 
The most detailed and complicated approach proposed by Noack et al. (2016) is the third, shown as " $\mathrm{C}$ " in Figure 40 with the original workflow used in Figure 51. Manual model manipulation and a different translator are used in place of the tools and processes mentioned.

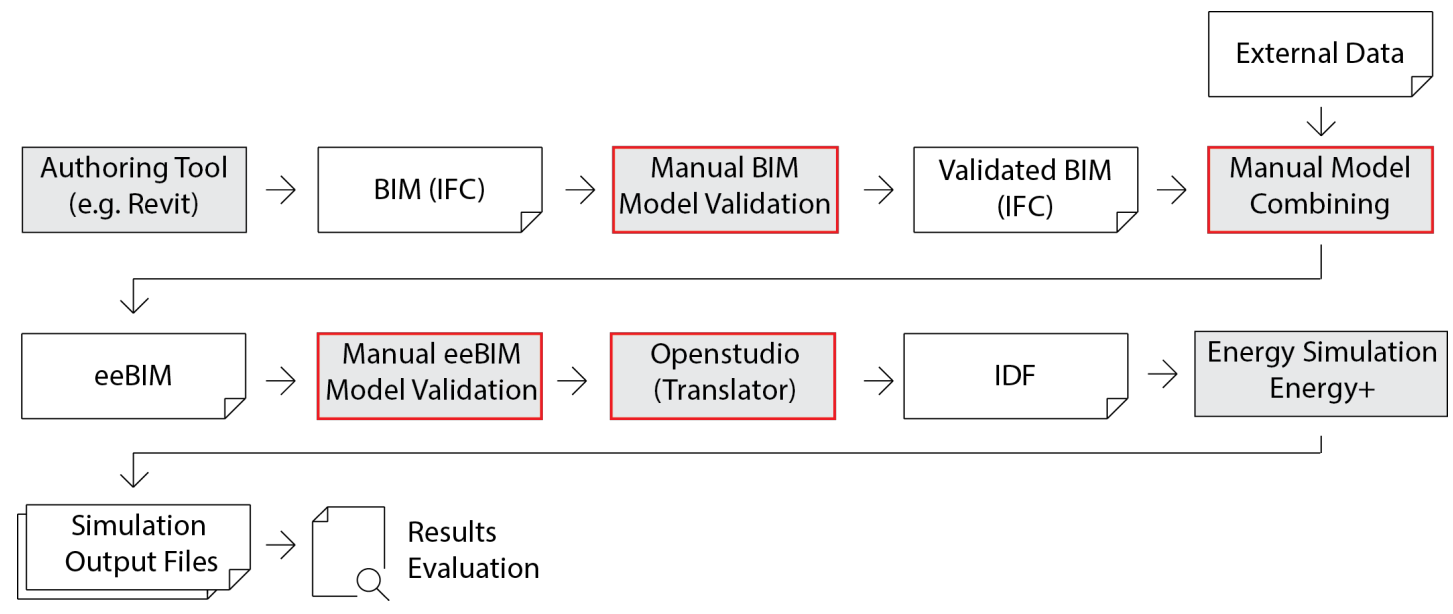

Figure 52: Adjusted Approach "C" from Noack et al. (2016)

Four steps were adjusted in Figure 52. These are the BIM model validation, model combining, eeBIM model validation, and the OpenStudio translator. The BIM validation step that cannot be performed automatically assesses whether the IFC output contains the relevant and required information. As IFC does not export everything that is required for energy simulations, external data must manually be added to the BIM model. Combining external data such as schedules, loads, set points, and material properties allow the BIM model to be identified and used as an eeBIM. The process involves stripping down an IFC file to only the information required for energy simulation by using an MVD. As no MVD is specified, external data is manually applied. OpenStudio is then used as the model translator because IFC files can be directly imported using the BIMserver. An IFC BIM file is exported from Revit and validated through manual inspection. The check is completed to identify whether there is specific geometry, loads, constructions, and construction properties in the IFC file. Determining that there is validates the export as a success for this stage. External data is then manually combined with the validated IFC to generate an eeBIM file. This process consists of adjusting the exported schedules to be consistent with what is required, as well as making sure that the loads are correct. Because IFC files generate their own unique IDs for everything stored in them, adding new information manually is complex. As there were not originally individual material properties, they cannot be added without model combining software, which is not accessible. Therefore, an eeBIM is not produced.

Although the low complexity models were produced there was no way found to continue the process. Meaning there was no failure, but also no success, so a not applicable rating is given. Due 
to the issues with testing of the low complexity model, the high complexity model is not attempted. Inaccessible problems would occur at the same junction.

\section{Process in Giannakis et al. (2015)}

The approach from Giannakis et al. (2015) contains adjustments for: manual replication of proprietary code or processes.

Table 40: Summary of Approach from Giannakis et al. (2015)

\begin{tabular}{|c|c|}
\hline Low Complexity & High Complexity \\
\hline Adjusted Process Failed & Adjusted Process Failed \\
\hline Manually Edited Process Successful & Manually Edited Process Successful \\
\hline
\end{tabular}

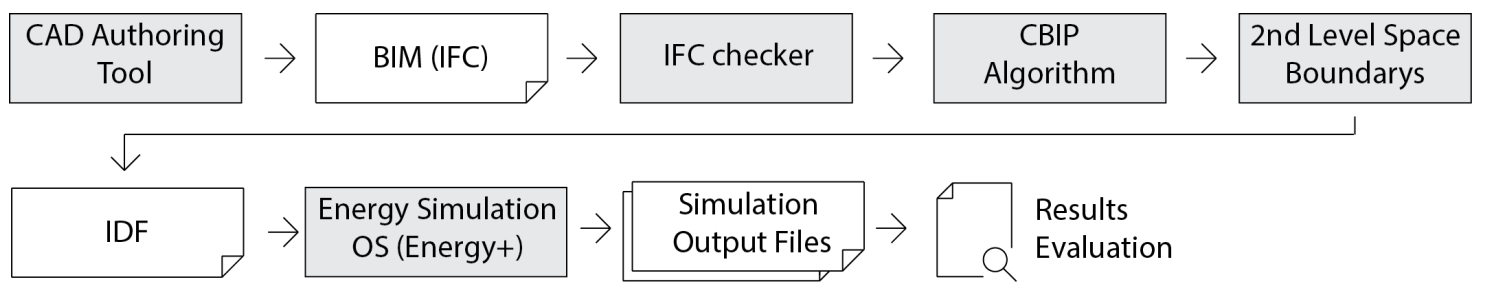

Figure 53: Approach from Giannakis et al. (2015)

The last process set out in Figure 39 and shown above uses an algorithm to translate IFC BIM information into an IDF format for simulation in EnergyPlus. It describes the algorithm as a CBIP that identifies boundary surfaces and intersects them accordingly. This is done so that secondlevel space boundaries are possible to generate. The boundaries and associated building information are translated through a program that has a defined ruleset. This process results in an EnergyPlus file capable of simulating a thermal environment and generating energy consumptions. Figure 40 shows the adjusted approach replaces the algorithm steps with OpenStudio for translation. It also replaces the previously ambiguous "CAD" with Revit. The CBIP algorithm and translation rules are described in Giannakis et al. (2015), but there is no way to implement them without obtaining the original script in far more detail.

The process fails for both model complexities tested due to a few issues that occurred. Manually editing to avoid or solve the issues allowed simulations to complete successfully.

\subsection{Energy Consumption}

\subsubsection{Low Complexity Model Energy Comparison}

Three distinct patterns were seen in the energy results of Figure 54. Firstly, that group one has identical total consumptions. Secondly, group three and four are identical. Lastly, the approach from Santos et al. (2017) is unique. These patterns arise because of the underlying processes used 
to produce them. Even though these approaches all originate from the same model the different process, manipulations, and issues applied to them alter the results.

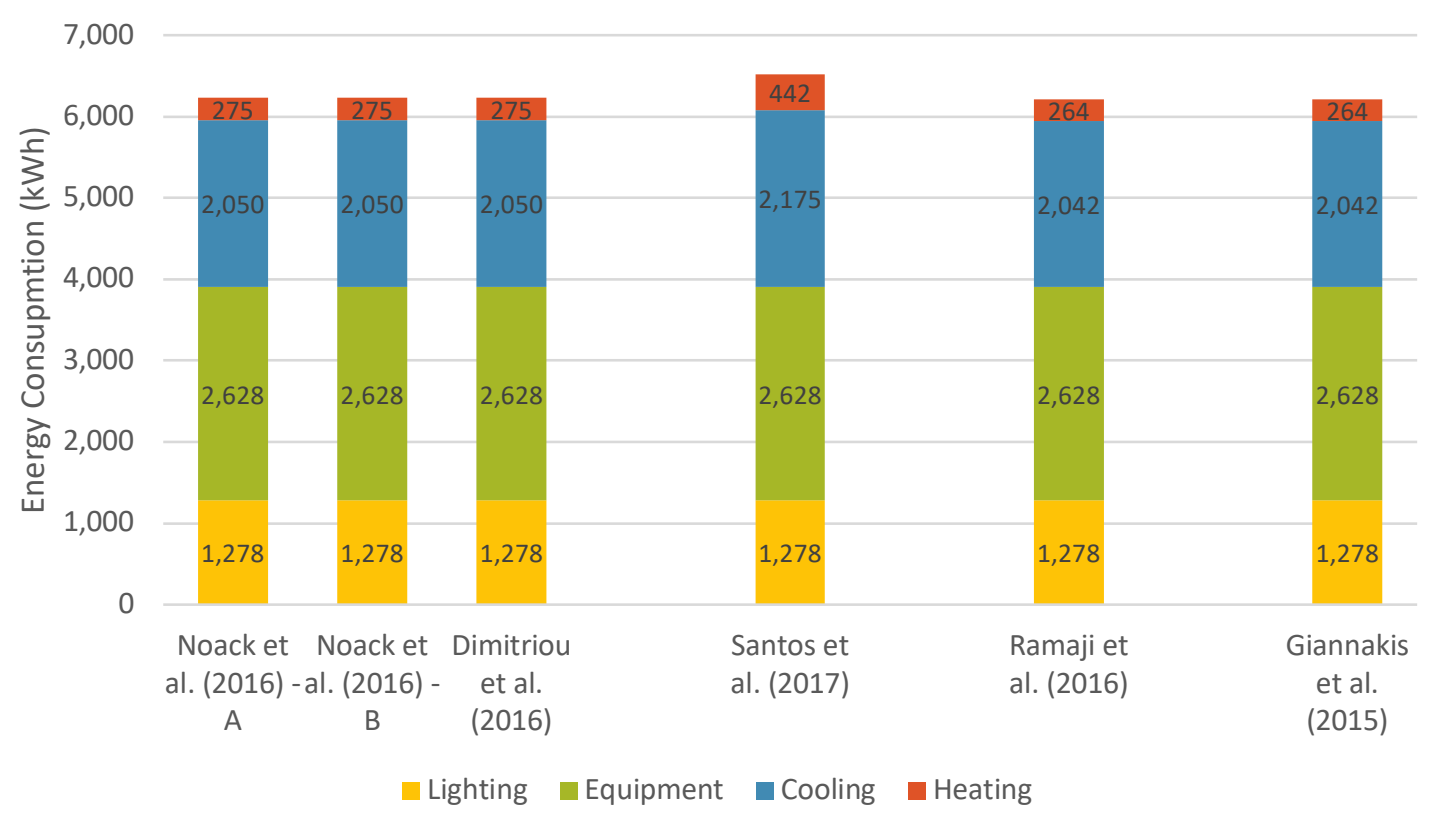

Figure 54: Energy Consumption Comparison of Low Complexity Model

The difference between all the approaches is $\pm 155 \mathrm{kWh}$, which equates an error of $2.5 \%$ of the average energy consumption. This error percentage is quite low, in part this is due to the static loads (lighting and equipment) being over $50 \%$ of the overall consumption.

The purpose of analysing the energy consumptions is to ensure that unnoticed errors are not making it through the process. If something were, it would display as vastly different to the other energy consumptions. This correction process is seen to work for the approach by Santos et al. (2017). It initially had questionable energy consumption patterns. The heating and cooling consumptions were opposite when compared to the other models. This caused a further investigation into the model, where it was noticed that natural ventilation and a different ideal loads approach is being used.

Each model is brought into OpenStudio and reviewed to determine what is causing the different energy consumptions. After inspecting the model's construction, materials, schedules, loads, and geometry, no major differences can be seen.

The $100 \mathrm{~m}^{2}$ low complexity model consumes an average of $63 \mathrm{kWh} / \mathrm{m}^{2}$ or $6,273 \mathrm{kWh}$ per year. This consumption falls below the expected value for an average household in Wellington of 10,860 $\mathrm{kWh} / \mathrm{yr}$. (HEEP, 2010). When compared to the average energy consumption per person and it is assumed that the smaller house contains two occupants; the estimated energy consumption is 
$7,240 \mathrm{kWh} / \mathrm{m}^{2} \pm 560$. The energy consumption does not fit within this range either, at $6 \%$ lower. Although the model does not fit within the HEEP data, it is not drastically different and is still a plausible energy consumption for a small residence.

Additional Quality Assurance to ensure the models are running correctly is completed by halving the insulation in all external elements. The cooling loads of each model decrease and the heating loads increase, as predicted in section 3.9.4. These results are explained in Figure 59 of Appendix Two. Interestingly, only in the approach by Santos et al. (2017) does the total energy consumption increase, whereas all other approaches consumption is decreased. This shows that within the approach something is not being correctly applied.

\subsubsection{High Complexity Model Energy Comparison}

As the simple model was to test, can the process be made to work, this model is to test can the process deal with a realistic model of a building.

The high complexity model used throughout the seven approaches originally has a floor area of $132.2 \mathrm{~m}^{2}$ as seen in section 3.9.1. Due to the complexity of the model, this is distorted through file translation. A floor area of $139.5 \mathrm{~m}^{2}$ is reported out of the energy simulation software for approach one, two, and seven. While, approach four and six show a slightly larger value of $141.6 \mathrm{~m}^{2}$ and approach five slightly less at $137.1 \mathrm{~m}^{2}$. These distorted values are not seen with the low complexity models in Figure 54, which means it is occurring because of the higher complexity. The lighting and equipment energy consumptions are also slightly differing shown in Figure 55 below because they are calculated based on area. The effected difference in just lighting and equipment is $\pm 88 \mathrm{kWh}$ or $1.6 \%$ of their total. This might seem small but it could influence the heating and cooling consumptions as well. The largest lighting and equipment consumptions do have the lowest heating and cooling. Nevertheless, this relationship cannot be proven as approach four and six had the lowest energy consumptions with the low complexity model, though at a drastically smaller percentage. 


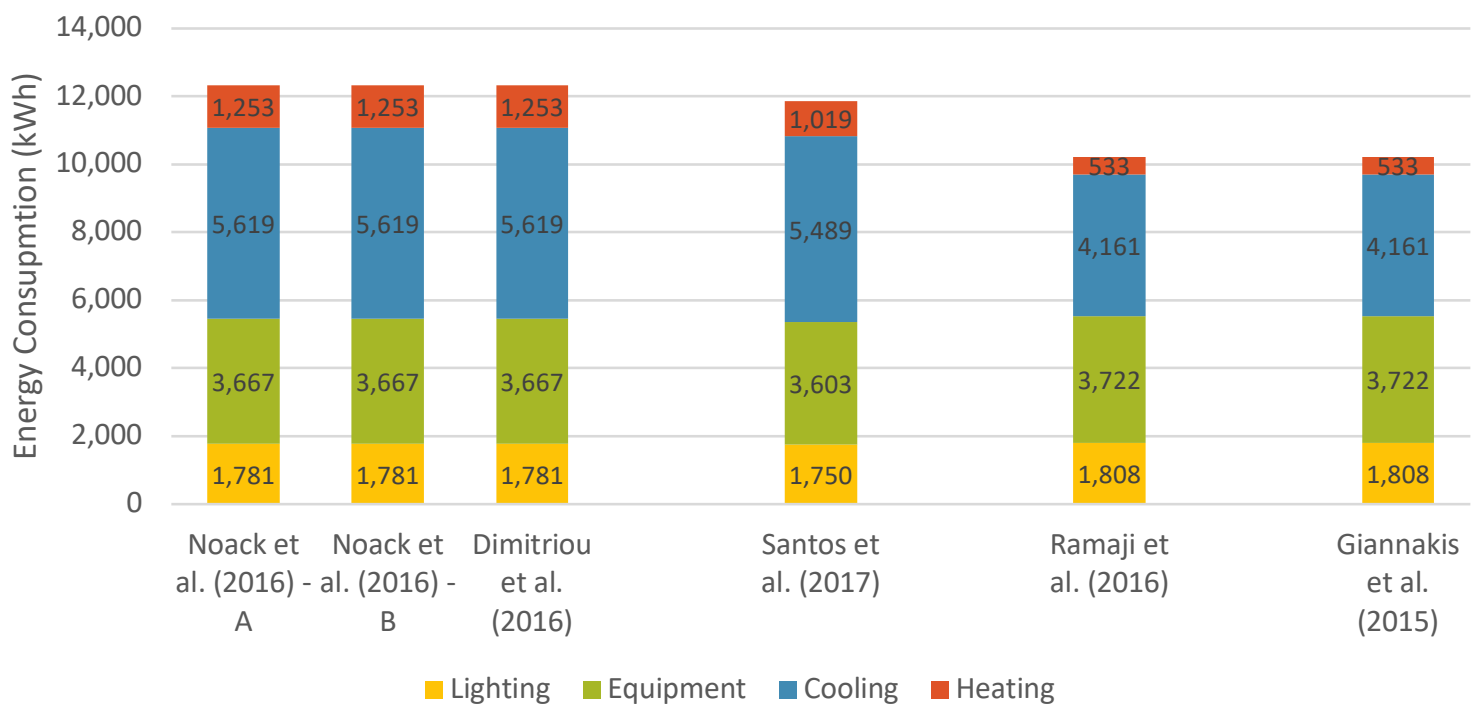

Figure 55: Energy Consumption Comparison of High Complexity Model

A large $\pm 1,047 \mathrm{kWh}$ difference in energy consumption is shown with the high complexity models. There is no obvious reasoning for this difference when the low consuming and high consuming models are compared.

With the differing floor areas, the average energy consumption of the complex models is $83 \mathrm{kWh} / \mathrm{m}^{2}$ or $11,545 \mathrm{kWh}$ per year. This value fits within the range identified in HEEP (2010) for Wellington of $10,860 \mathrm{kWh} \pm 790$. This Quality Assurance step shows that the energy consumption of the models is within a credible range. Further Quality Assure is provided by the assessment discussed in section 3.9.4 where insulation values are reduced by $50 \%$ to ensure that the software reflects what would be expected. Figure 60 in Appendix Two identifies that this is true through the increase in heating load and decreasing in cooling load. 
Chapter Four: Testing 


\section{Chapter Five: Results}

In the previous chapter, the processes are tested with a low and high complexity Architectural models. Testing brings the answer to if BIM translations processes are already available, can they be used by Architects.

The issues identified through testing are visualised in this chapter by showing the stages of failure and success, the number of issues identified, and what issues each process found. While there are no clear divides between what process should be used over another, there are evidently processes with more apparent issues. However, just looking at the number of issues is not enough to quantify how prone a process is to failure, as certain issues are more complex, taking more time and effort to solve for. 


\subsection{Stages of Progress}

There are three distinct stages that all identified process contain, these stages being export, Middleware, and simulation. Export is the process of generating a readable file that contains not only building geometry, but also other properties required for BPS. Middleware is the process of taking said exported file and reformatting it to be readable by an energy analysis program. Lastly, the simulation stage is when an analysis software is used to produce energy or other BPS results from the input.

A comprehensive comparison of each simulations points of failure and successes are displayed in Table 41. All the unedited processes tested with a low or high complex model fail in some way, either at the Middleware or simulation stage. Unsurprisingly, most tests fail while the model is being simulated; this is mostly due to small but important pieces of information missing. Information is lost in the Middleware translation stage a high proportion of the time. Interestingly, only one process contains a partial export while the others tested are all successful. For the most part, information is correct in the export process; however, the main downside is not all the required information is present.

A major downside of many processes is the level of depth in describing how they work. This is shown through the comparison of edited vs unedited. When the proposed processes are edited, almost every stage is successful, and overall the process works better. This is because the identified issues can be solved manually. These edits are completed by the author, who has experience and expertise around finding and solving errors, and the general use of BPS, whereas the expected audiences, Architects would not have this understanding.

The differences in successful completion are minor between the low and high complexity models.

Table 41: Points of Failure and Success

\begin{tabular}{|c|c|c|c|c|c|c|c|}
\hline $\begin{array}{l}\frac{\pi}{\varepsilon} \\
\frac{\delta}{U} \\
\frac{\mathcal{U}}{\sim}\end{array}$ & 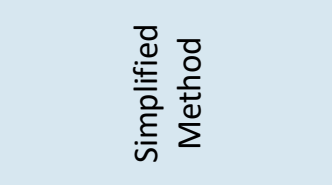 & 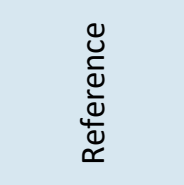 & $\begin{array}{l}\frac{\vec{x}}{x} \\
\frac{0}{0} \\
\frac{0}{\varepsilon} \\
0\end{array}$ & 导 & $\begin{array}{l}\text { tٓ } \\
\frac{0}{2} \\
\frac{0}{x}\end{array}$ & $\begin{array}{l}\frac{0}{\frac{1}{0}} \\
\frac{0}{0} \\
\frac{0}{0} \\
\frac{0}{2}\end{array}$ & $\begin{array}{l}\frac{\circ}{0} \\
\frac{0}{0} \\
\frac{\pi}{5} \\
\frac{E}{n}\end{array}$ \\
\hline \multirow{9}{*}{$\underset{\substack{x \\
\infty}}{\vec{\infty}}$} & \multirow{9}{*}{$\begin{array}{c}\text { gbXML } \rightarrow \text { OpenStudio } \\
\rightarrow \text { EnergyPlus }\end{array}$} & \multirow{4}{*}{$\begin{array}{c}\text { Noack et al. } \\
\text { (2016) }\end{array}$} & \multirow{2}{*}{ Low } & No & & & \\
\hline & & & & Yes & & & \\
\hline & & & \multirow{2}{*}{ High } & No & & & \\
\hline & & & & Yes & & & \\
\hline & & \multirow{4}{*}{$\begin{array}{c}\text { Noack et al. } \\
\text { (2016) }\end{array}$} & \multirow{2}{*}{ Low } & No & & & \\
\hline & & & & Yes & & & \\
\hline & & & \multirow{2}{*}{ High } & No & & & \\
\hline & & & & Yes & & & \\
\hline & & & Low & No & & & \\
\hline
\end{tabular}




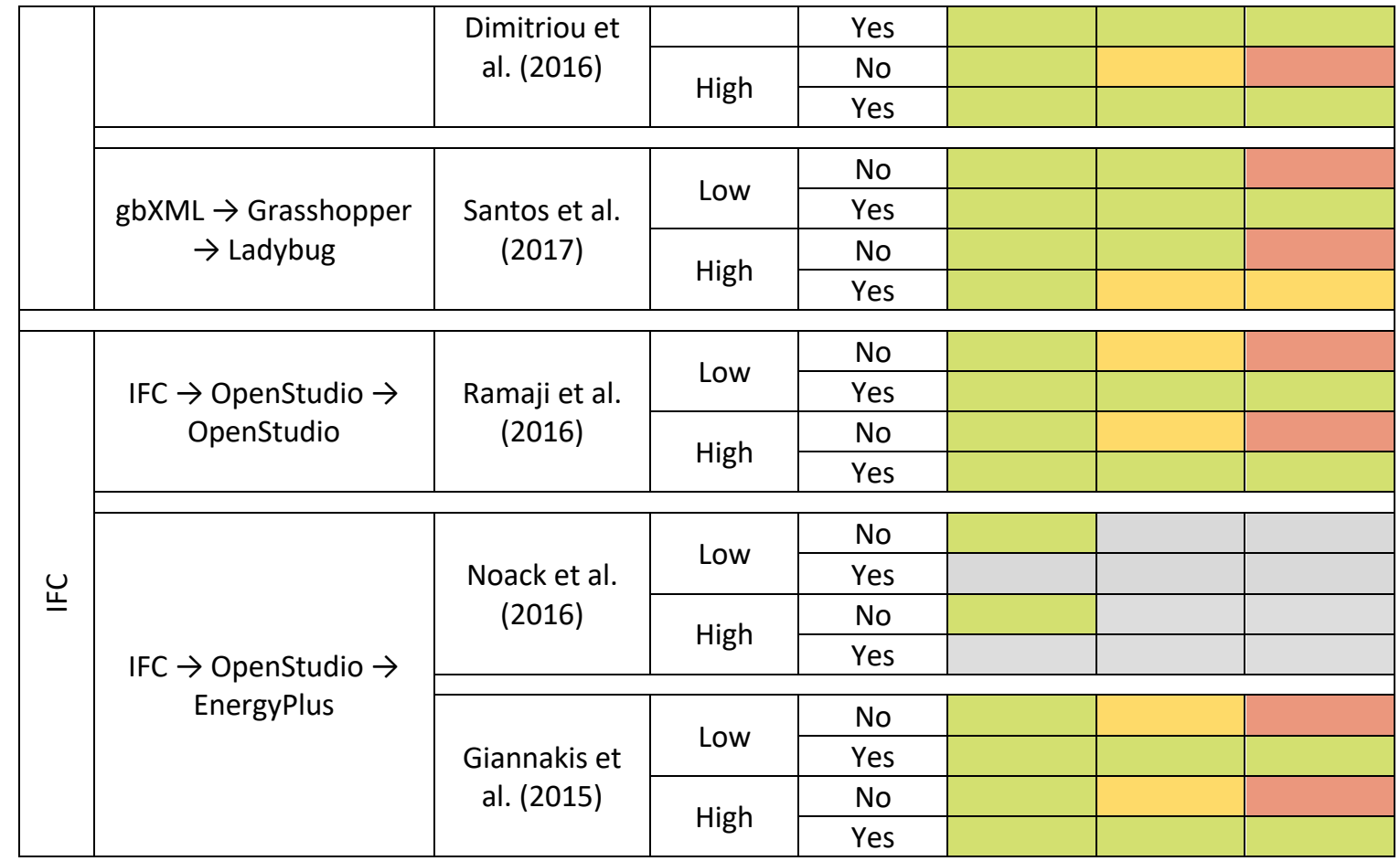

\begin{tabular}{|c|c|}
\hline Successful & \\
\hline Partial & \\
\hline Failure & \\
\hline n/a & \\
\hline
\end{tabular}

\subsection{Number of Issues Identified from Testing Approaches}

Testing of the processes established 55 individual issues across nine categories in Figure 56. The low complexity model highlighted 27 issues, and the high complexity model 28 . Schedules, loads, and weather and location were found to be the most commonly identified issues.

Loads not translated into BPS

Schedules not translated into BPS

Weather properties and location not defined

Materials properties not defined individually

Surface Matching of internal elements are incorrect

Surface normal incorrect, orientation is flipped to external

Airflow and infiltration incorrectly applied

Space names of zones are duplicated

Unknown issue that causes failure couldn't be identified
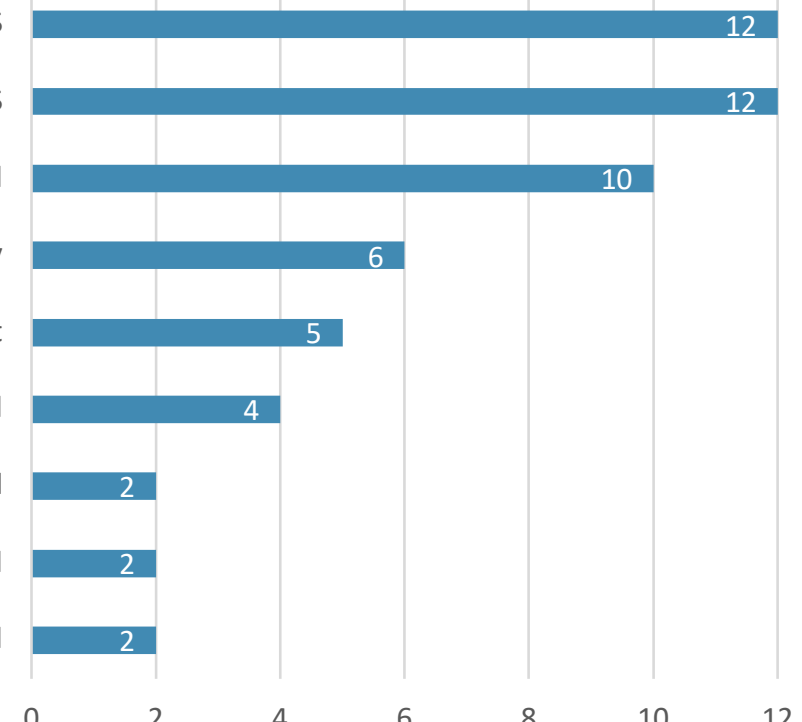


\section{Figure 56: Quantity of Issues Identified}

Loads: Building operation is not translated from Architectural models when they are created. Some values tangentially related to loads, set points, are exported if specifically set. However, these are not read directly into analysis software, highlighting an inconsistent process.

Schedules: Schedules are related to loads as they each require information from the other to work correctly, therefore, the same quantity is identified. Schedules are exported some of the time into the corresponding format, yet, they are not importable into BPS due to the formatting.

Weather and Location: Issues around weather and location are mostly caused by there being no explicit way, or step to apply such information. Normally this data could easily be applied as a weather file, which contains all of the properties and location data to support simulation. No weather data or location information is exported from Architectural modelling software into the file formats used.

Materials: The properties of materials are not always exported, and aren't always exported correctly either. Properties such as resistance, density, and specific heat capacity, not commonly used in Architectural practice can be embedded in some file schemas from the software used originally.

Surface Matching: This process is not always completed fully or even at all. Internal doors and ceilings to floors are often the elements that don't get automatically matched.

Surface Normal: Along with not surface matching, internal elements sometimes reverse the normal direction in which they face, meaning external surfaces are facing inwards. This creates confusion in BEM that does not allow model to simulate correctly.

Airflow: Differing infiltration and ventilation rates are automatically applied through certain methodologies causing issues.

Spaces: Duplicate space or zone names in exported files causes both occurrences to be removed when reading into the analysis software.

\subsection{Breakdown of Issues Identified from Each Approach}

The types of issues identified differ depending on schema and process used. Laid out in Table 42, the schema used divides the table once, and it is divided again based on how the methodology works when simplified. Processes using one of the more simplistic approaches of "gbXML $\rightarrow$ OpenStudio $\rightarrow$ EnergyPlus" contain the most common issues highlighted in Figure 56. Surprisingly, the first process is the only completed process to not pose any issues with weather and location. 
The second process using gbXML files produced a few more errors that than the first set, including another unique issue relating to airflow. Processes using IFC as the schema seem to consistently comprise of the simpler issues along with less common issues.

Table 42: Issues Identified from Each Approach

\begin{tabular}{|c|c|c|c|c|c|c|c|c|c|c|c|c|}
\hline & 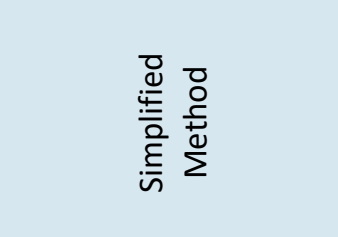 & 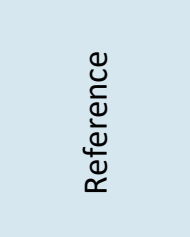 & $\frac{\frac{\partial}{x}}{\frac{1}{0}}$ & $\begin{array}{l}\text { ñ } \\
\text { D } \\
0\end{array}$ & $\begin{array}{l}\frac{\tilde{d}}{5} \\
\frac{0}{0} \\
\frac{\tilde{U}}{u}\end{array}$ & 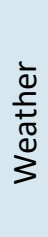 & 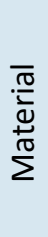 & 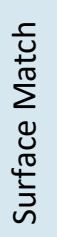 & 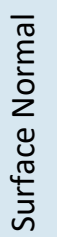 & $\frac{3}{\frac{3}{E}}$ & 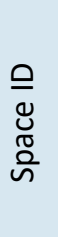 & $\begin{array}{l}5 \\
\vdots \\
0 \\
\frac{5}{5} \\
\frac{5}{5}\end{array}$ \\
\hline \multirow{8}{*}{ 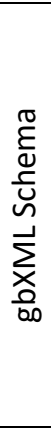 } & \multirow{6}{*}{$\begin{array}{c}\text { gbXML } \rightarrow \text { OpenStudio } \\
\rightarrow \text { EnergyPlus }\end{array}$} & \multirow{2}{*}{$\begin{array}{c}\text { Noack et al. } \\
(2016)\end{array}$} & Low & & & & & & & & & \\
\hline & & & High & & & & & & & & & \\
\hline & & \multirow{2}{*}{$\begin{array}{c}\text { Noack et al. } \\
(2016)\end{array}$} & Low & & & & & & & & & \\
\hline & & & High & & & & & & & & & \\
\hline & & \multirow{2}{*}{$\begin{array}{l}\text { Dimitriou et } \\
\text { al. (2016) }\end{array}$} & Low & & & & & & & & & \\
\hline & & & High & & & & & & & & & \\
\hline & \multirow{2}{*}{$\begin{aligned} \text { gbXML } & \rightarrow \text { Grasshopper } \\
& \rightarrow \text { Ladybug }\end{aligned}$} & \multirow{2}{*}{$\begin{array}{c}\text { Santos et al. } \\
(2017)\end{array}$} & Low & & & & & & & & & \\
\hline & & & High & & & & & & & & & \\
\hline \multirow{6}{*}{ 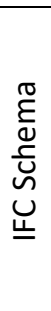 } & \multirow{2}{*}{$\begin{array}{c}\text { IFC } \rightarrow \text { OpenStudio } \rightarrow \\
\text { OpenStudio }\end{array}$} & \multirow{2}{*}{$\begin{array}{l}\text { Ramaji et } \\
\text { al. (2016) }\end{array}$} & Low & & & & & & & & & \\
\hline & & & High & & & & & & & & & \\
\hline & \multirow{4}{*}{$\begin{array}{c}\text { IFC } \rightarrow \text { OpenStudio } \\
\text { EnergyPlus }\end{array}$} & \multirow{2}{*}{$\begin{array}{c}\text { Noack et al. } \\
(2016)\end{array}$} & Low & & & & & & & & & \\
\hline & & & High & & & & & & & & & \\
\hline & & \multirow{2}{*}{$\begin{array}{c}\text { Giannakis } \\
\text { et al. (2015) }\end{array}$} & Low & & & & & & & & & \\
\hline & & & High & & & & & & & & & \\
\hline
\end{tabular}

Not demonstrated in Table 42 is how the different model complexities take considerably different amounts of time to edit and solve the issues. Specifically, issues relating to surface match, and surface normal. This is because the high complexity model contains almost 10 times as many surfaces, whereas only containing just over four times the number of zones, presented in Figure 57. These presumably linear increases highlight issues that could occur just in terms of documenting all of the information. With IFC, the low complexity model is contained in 494KB of data, while the higher complexity model within 4,084KB. If a large commercial building is required to be analysed these would be expected to be much larger. 


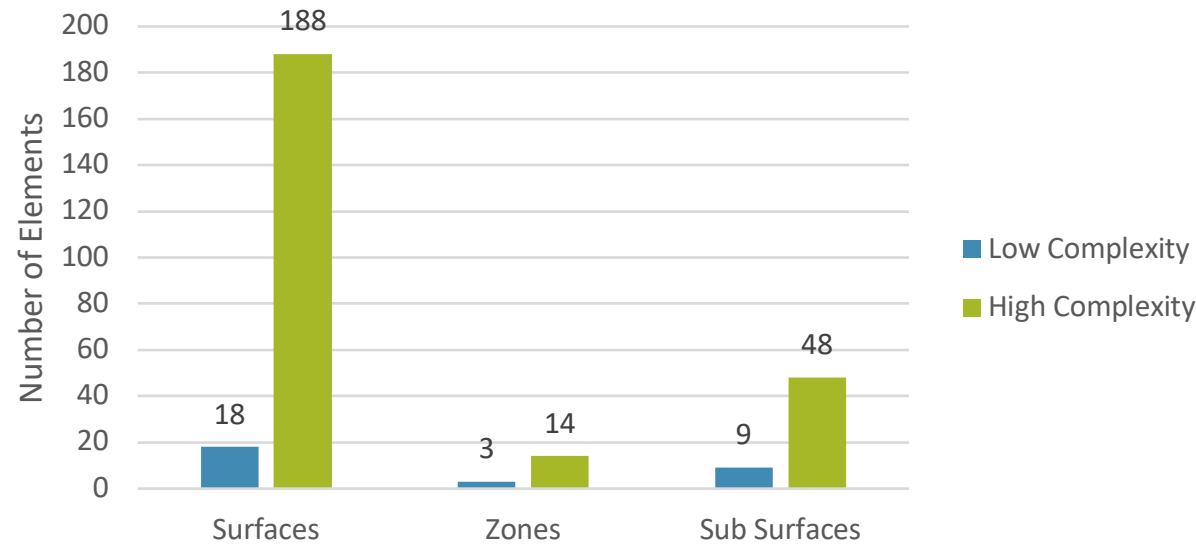

Figure 57: Model Breakdown

The surface to zone ratio in Figure 58 shows how the two models' complexities drastically vary. On average each zone in the lower model contains just six surfaces, which is the minimum for a completely sealed rectangular volume. Compared to the average of 13.4 surfaces per zone in the higher complexity model. This higher value is due to different shaped zones, surface matching to surrounding zones, and surfaces for differing wall makeups.

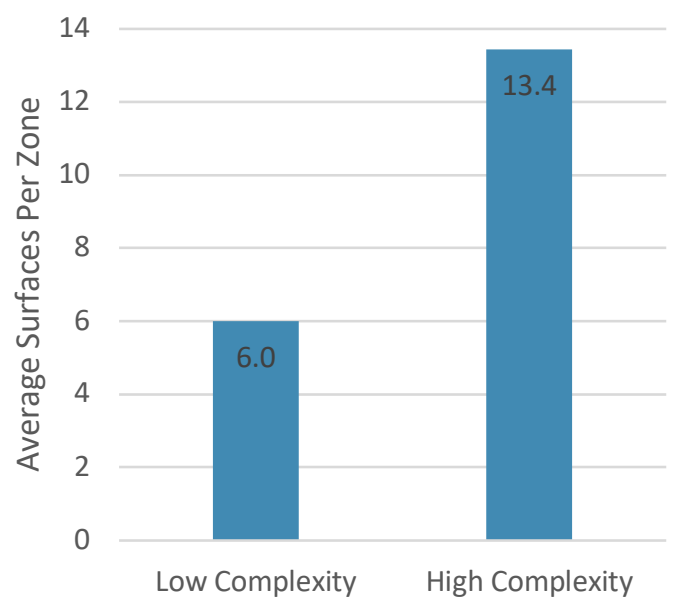

Figure 58: Ratio of Surfaces per Zone in Models

Models become more complex with more spaces, stories, and windows, meaning manual solutions to issues become unfeasible. An estimate of the time taken to resolve surface matching in the low complexity model is ten minutes, while for the high complexity model it would be closer to one and a half hours. Because the intended audience is Architects, who are already busy, this increased time to solve a single issue is unfeasible and could be considerably larger with an even more complex model. 
Chapter Five: Results 
Chapter Five: Results 
Chapter Six: Discussion

Chapter Six: Discussion

This chapter presents the discussion of 
Chapter Five: Results. While there is a large body of research that supports the improvement and development of Architect friendly tools aimed to integrate with BPS tools (Alsaadani and Bleil De Souza, 2016, Attia et al., 2012). The problem that is not identified in that body of research is that programs designed to translate building information fail a high proportion of the time, as seen in Table 41. These approaches are discussed below. 


\subsection{Points of Failure and Success}

\subsubsection{Category One: gbXML $\rightarrow$ OpenStudio $\rightarrow$ EnergyPlus}

The first process produced a valid model with correct geometry and constructions. However, energy results cannot be produced without the manual implementation of schedules and loads. As from the identification of issues section 3.4.1, many problems are related to geometry and materials, so for them to translate is positive. Table 41 identifies the approach failed at the simulation stage due to only a partial translation of information. When the required loads and schedules are re-applied correctly, all three key steps of the process are successful and the model produces energy consumption results. The high complexity model also failed during the translation stage, due to duplicate space IDs and no schedules or loads. It is the only process to have a partial export, which is caused by ceilings not being recognised. When these issues are manually solved during the process, the model exports, translates, and simulates successfully.

With no way to apply the required schedules and loads without manual implementation in OpenStudio, approach "B" from Noack et al. (2016) shows no improvements over approach "A". Manually applying the missing properties allows the simulation to run successfully. When accessing approach " $\mathrm{B}$ " with the complex model, a step to adjust the exported code is used, the duplicate text is removed allowing for a successful step in comparison to approach " $A$ ". The adjusted process applies schedules and loads within OpenStudio that were causing a partial translation in the original method. The combination of these steps allows the model to be successfully simulated and results produced.

The third process, in Dimitriou et al. (2016) is also found to have a partial Middleware translation due to information not being exported or even implemented into the Architectural model. This then causes a failure when an incomplete model is simulated. The adjusted approach successfully passes through the three steps once the identified issues are solved. As with the low complexity model, the high complexity model using this process also fails during the simulation stage. This is due to errors and issues that occur in the translation section.

\subsubsection{Category Two: gbXML $\rightarrow$ Grasshopper $\rightarrow$ Honeybee}

The approach in category two using processes from Santos et al. (2017) is initially tested with complex curved geometry, and could not be completed, due to the lack of information provided on the approach in terms of planarization. This meant a generic planar model could be tested. As the model is generic the energy consumption is irrelevant, but it does show the potential of the process to generate and display calculations over a model. The model translation is from non- 
planar to planar, as this step could not be completed the translation fails and so does the simulation. With manual adjustments to make a planar model, the workflow completes the simulation successfully. This test shows the potential benefits for a similar approach, but also the complexities of geometry planarization.

For the low complexity model to be successful, two additional steps are implemented. As Architectural modelling software ArchiCAD and Revit do not allow double-curved geometry to be generated, it is a safe assumption that flattening the geometry of models would not be needed. However, without the schedules and loads that are not imported through the gbXML import component, simulations are not possible. The same approach with the addition of manually applying schedules and loads the EnergyPlus simulation runs correctly and produces an output. More information must be used to run the simulation and ensure the same variables as other models. These are manually constructed and applied to the model within the Grasshopper environment. It is a time-consuming task to generate these the first time, but they are then stored within the Honeybee file directory and can be re-applied to other models. This process proves how alternate material properties or constructions can also be applied. With all required properties applied, an EnergyPlus simulation can be run and results are analysed. Testing with the complex model fails during simulation, however contrary to previous processes the Middleware translation is completed successfully. The failure occurs because other schedules and loads that are not present in the original Architectural model are not available. Once schedules and loads are applied, and the other issues are solved the adjusted process still does not produce results without errors.

\subsubsection{Category Three: IFC $\rightarrow$ OpenStudio $\rightarrow$ OpenStudio}

Processes in Ramaji et al. (2016) use the file format IFC, it highlights failures at the same points as previous translation processes. This shows that specific problems are not directly related to which file schema is implemented. Table 41 highlights that with manual adjustments, the Middleware translation becomes successful, and that in turn allowed for a successful simulation. The factors that needed to be adjusted include, applying material properties, correcting surface normal, correcting surface matching, and the previously identified lack of schedules and loads. Using the high complexity model fails once again as with the low complexity model, due to poor translation of information. For a successful approach to take place, additional information such as schedules, loads, and material properties is applied to the model in OpenStudio to enable a complete simulation. A higher level of detail model means that the alterations required are equally more complex and time-consuming to perform manually. Having more internal doors and walls requires 
more surface matching to occur, in addition, a lot of surfaces have incorrect surface normal which must be reversed. Even though the adjustment process is more complex, it is completed and a successful simulation is performed in OpenStudio.

\subsubsection{Category Four: IFC $\rightarrow$ OpenStudio $\rightarrow$ EnergyPlus}

The first process in category four, from Noack et al. (2016) identified issues through testing that is not specific to file translation, but that the process itself. The amount of alteration that is done to make the process work is high, and it still could not be completed. An exported Architectural model is the only successful step in the process, as translation and simulation could not be repeated. With the complex model, the original approach only had one successful process, the export, and then the workflow could not be followed properly meaning the translation and simulation steps in Table 41 cannot be assessed.

The failure and successful points of the last approach from Giannakis et al. (2015) are set out in Table 41. A pattern of partial translations causing failures in the simulation stage is shown again like with most other approaches. The model performed identically to a similar methodology in category three. The only differences seen in Figure 40 are the simulation tools and an additional file transformation. The large range of issues identified to cause the Architectural models file translation into OpenStudio to be incomplete, which then does not allow any simulations to take place. Using the high complexity model the process fails. The failure occurs when the simulation is attempted to take place because of a partial translation of information out of the Architectural model. Once the identified issues are manually solved, each step is successfully passed and energy results produced.

\subsection{Identified Issues}

Notably the most abundant of the identified issues related to schedules, loads, and weather. They are recognized the most with 12,12 , and 10 occurrences respectively in Figure 56 . This is because they translate very poorly, and could not all be defined within the original Architectural software. Although these issues are produced the most often, they are also simple errors to solve either manually through OpenStudio or automatically though Grasshopper and Honeybee. Moreover, no simulation can run without them so it is obvious when one or more are missing, as the simulation process fails. The six occurrences of materials causing issues line up with the six processes using IFC as the translation schema. This problem is identified previously in section 3.4.1, however, it was expected that similar issues with materials would additionally be generated when using gbXML. Some of the more complicated issues with surface normal, surface matching, and airflow are only produced once or twice, because of the specific requirements that are needed for them 
to occur. The simpler and easier to understand issues are acknowledged most, while the more complex and intensive issues to solve and found less often. The importance of solving each issue can be ranked by how frequent it occurs, or by how long it would take to solve. In each case, the answers would be different and would likely start from each end of Figure 56. Issues with space ID are not identified with the low complexity model in Figure 56, while it is seen twice using a complex model, because it comes about when spaces are stacked vertically. This issue can occur in all processes that use the complex model and generate building geometry to be translated into gbXML. Another difference between the two complexities is that issues due to surface matching occur twice on the low complexity model, and three times with the high complexity model. This is because surface matching the complex model in Grasshopper could not be completed automatically.

\subsubsection{Category One: gbXML $\rightarrow$ OpenStudio $\rightarrow$ EnergyPlus}

Issues are identified through testing the process in approach " $A$ " from Noack et al. (2016) when using a low complexity model. The schedules and loads applied in the Architectural modelling software are not imported into OpenStudio, as seen in Table 42. However, it is interesting that the issues are not present in the IDF export from the model. This suggests that the format schedules are presented in, are an incorrect format for OpenStudio to read. For the processes to simulate successfully with a low complexity model, correct loads and schedules must be re-applied to the model in OpenStudio. This would have to be done manually in the OpenStudio software, or automatically to the OSM file. Testing of this approach with the high complexity model highlights some of the known issues with gbXML translation, being element duplication. In this case, ceilings and air walls are not generated due to the software thinking that if there is a duplicate space ID reference, the solution is to ignore both representations. This issue is created during the export process from Revit and is propelled by the Middleware not automatically understanding what should be done. Like with the low complexity model, schedules and loads are also translated incorrectly into the analysis software. This is expected because nothing has altered in the process apart from the test model becoming more complex. The other issue exposed is that not all zones represented in Revit are exported individually, but as a whole. The solution to getting the process working is to remove duplicate text from the gbXML export using a text editor manually; this could be set up to run automatically. In addition to, applying relevant schedules and loads.

Approach " $\mathrm{B}$ " identified issues through the testing of the second set of processes proposed by Noack et al. (2016). Table 42 shows that the issues found are the same as in approach " $A$ ", meaning the adjusted process does not allow schedules or loads to be understood. However, this 
process does not specify, when or even that a weather file is required for simulation. The solution, as above, is to manually create and add them to the OpenStudio model. The issues identified through the testing of approach " $\mathrm{B}$ " with a high complexity model are just around schedules and loads, like the other processes tested by Noack et al. (2016). This methodology is an improvement over what is suggested for the first approach. Because of editing the gbXML to remove unneeded information, the space ID issue does not appear. Figure 56 shows that the issues identified with approach two are the most common, found in almost all the other processes.

The third approach in this category is shown to identify similar issues to the other processes. The issues being weather data, schedules, and loads. This is expected, as the adjusted approaches in Figure 40 are similar. These three issues are the most common to appear. The higher complexity model identifies these issues and an additional issue with space ID's. This approach found an average amount of issues compared to the other approaches in Table 42. Space ID, which is only identified in one other approach, is caused by duplicate space name text being produced and then the original and duplicate are ignored.

\subsubsection{Category Two: gbXML $\rightarrow$ Grasshopper $\rightarrow$ Honeybee}

The test from Santos et al. (2017) displays a limitation in relation to the exact processes used, as it is not clearly defined. An educated approach is taken as to how it would work within Grasshopper. There are clear benefits to simulating a model in this software; if a BIM model can be imported instead of a model, being directly created in Rhino this process has large potential benefits to surpass some of the identified issues. There are no issues identified through testing in relation to building information translation. However, the one issue that does occur is because of the non-planar geometry that the workflow promotes as being able to handle.

The low complexity model fails with the original processes due to schedules and loads not being imported and applied. The solution that is used to get the process working is to apply them manually in Grasshopper using the VPL interface in Ladybug. This initially allows the model to run successfully, but it does not seem to produce correct results. Inspecting the model in OpenStudio highlights the other issues in Table 42, related to materials and airflow. The internal and external doors are not being recognised as doors and have glazing materials applied to them. This is simple to solve within OpenStudio but cannot be adjusted within Grasshopper. This adjusts energy consumptions slightly, but they are well below what other modes are consuming and unexpectedly opposite in terms of consumption pattern. The other issue related to air is discussed because two settings are automatically assigned when using Grasshopper. These are the design specification for outdoor air, and space infiltration flowrate. Having not been set on other models, 
they are removed to have a fair variable free comparison. The ideal load settings are also different. Transitioning the model though Grasshopper creates a set of zone equipment named ideal air loads, however, it does not perform identically to the ideal air load switch in OpenStudio. When these modifications are made the model produces energy consumptions closer to the other models shown in. The high complexity model reveals other issues that either originate in the modelling tool or occur during Middleware translation. Three zones in the model are not able to generate an internal volume because the surrounding surfaces do not fully enclose the zone. This is caused by duplicate geometry both being removed during Middleware translation or incorrect modelling causing a gap in the model. As Revit provides an analysis, option when exporting the analytical model to gbXML it can be ensured that there are no gaps in the model. Other problems, which would cause altered results, come from surfaces interacting incorrectly with each other. The backside of some surfaces is in front of the receiving surface during the interior solar distribution calculation. The cause cannot be identified. The high consumption in the highlighted zone of Figure 49 is caused by the floor being considered a skylight. As in the Architectural model, a staircase is passing through this zone and the one below it, the analytical model determines that glazing should be used instead of either combining the zones or creating an air wall between them. This issue then propagates, as the energy simulation program cannot find any geometry defined as floor causing errors in the calculation process. Although errors are seen, the energy consumption is within the realm of reality shown in Figure 55.

\subsubsection{Category Three: IFC $\rightarrow$ OpenStudio $\rightarrow$ OpenStudio}

The only approach in category three highlights equally the most issues of all processes tested. This may be due to the IFC schema containing a lot more potential issues than other schemas, as identified in section 3.4.1. In this process from Ramaji et al. (2016), six problems are identified. The initial issue is that a weather file is not specified during the process; therefore, it is not translated with the Architectural model. A major issue is that ceilings cannot be translated if they are external, due to there being no IFC identifier for them. Because energy models are produced using space zoning within an Architectural model, whatever is intersecting the top space surface is converted into the IFC export. The solution can either be, increase the zone size to intersect with the roof geometry, or to change the ceiling properties to be a roof. In this case, the second approach is used so that the space volume is more accurately reflected. The second issue, which is also identified to occur with gbXML translation, is the missing loads and schedules. These must be re-applied in OpenStudio for simulations to run successfully. Unlike gbXML translations, the third issue is to do with material properties not being translated correctly. Default values are applied for each material in a construction even though the whole construction resistance is 
present in the IFC. When they are translated the property order is also mismatched, this can cause inaccuracies during the simulation as the layer order plays a part in thermal, visual, and solar Absorptance properties. The fourth issue is identified when the simulation takes place, and sever errors occur. IFC export from Architectural software does not contain boundary surface properties for internal surfaces. Therefore, internal walls, doors, floors, and windows are incorrectly represented as external surfaces, some of which are even facing the wrong direction, which causes simulation failure. To solve this issue some surfaces must be manually reversed and then surfaced matched to the corresponding element. While this issue can be quick to identify, it is a considerably more complex issue to fix. The final problem identified through this process is scale. The imported model is translated as if it was exported when the document units are set to meters. Therefore, when OpenStudio imports the model it assumes the model's dimensions are in millimetres. Making dimensions a thousand times out. To solve this the IFC model requires reexport with the correct units.

To use this approach, the low complexity model is simply exported from Revit in the IFC schema. The exported IFC file contains geometry, loads, constructions, and construction properties, in addition to missing schedules. Importing an IFC to BIMserver is completed through the OpenStudio interface. Once imported, the translated OSM can be downloaded and opened directly into OpenStudio. All geometrical surfaces are imported apart from the roof. This is identified to be because of IFC not containing an identifier for ceilings, which is what the extents of the space in Revit, is intersecting. The IFC schema identifies different parts of geometry by naming them as in Table 49 in 
Appendix Three.

As importing ceilings is not possible, the model is altered to contain a roof. This adjustment allows for the geometry in terms of surfaces to be imported. A few other sets of information that should have been contained in the original IFC are missing from the translated OSM including loads and construction properties. Loads are non-existent and there is no reference to them in OpenStudio. However, there are construction properties in the file they are just incorrect. Figure 63 in 
Appendix Three, shows how the external wall construction set is imported, the constructions are correct but in the incorrect order, while the properties are exaggerated and incorrect. This means that any resulting simulation is in turn incorrect. The process fails at the simulation stage due to the lack of some required information. It is successful for obtaining geometry out of an IFC file; however, what is not converted from either Revit to IFC or IFC to OSM limits the potential.

To make the approach work manual adjustments need to be completed. Within OpenStudio, schedules, loads, and corrected material properties are applied. The simulation is attempted, but fails due to the orientation of internal surfaces and the surface matching of sub surfaces. In energy simulation, internal geometry must intersect at orientations $180^{\circ}$ apart, so that the back faces of each space meet. All internal surfaces are checked to ensure that the surface normal values are correctly assigned, any that are wrong, are reversed. Additionally, the internal surfaces and subsurface do not have a boundary surface which to be surface matched to defined, these must be manually re-applied. Table 50 in 


\section{Chapter Six: Discussion}

Appendix Three ascertains the surfaces that are generated with incorrect boundary surfaces.

After the adjustments are implemented, the simulation runs correctly, however, the results are exponentially larger than expected. As can be laid out in Table 51 of 
Appendix Three, the floor area of $100,000,000 \mathrm{~m}^{2}$ is larger than the originally input $100 \mathrm{~m}^{2}$. This is caused by the IFC model exporting using units that are set to $\mathrm{mm}$. Consequently, the $10,000 \mathrm{~mm}$ by $10,000 \mathrm{~mm}$ floor plan is read by OpenStudio as $10,000 \mathrm{~m}$ by $10,000 \mathrm{~m}$. Exporting a new-scaled IFC BIM solves the issue. Adjusting for all the other steps above the adjusted model runs successfully.

Issues with using a high complexity model are identified as the same as those with the low complexity model. However, the increased complexity meant far more time needed to be spent resolving the issues and implementing the solutions.

\subsubsection{Category Four: IFC $\rightarrow$ OpenStudio $\rightarrow$ EnergyPlus}

The last approach contains six issues as displayed in Table 42. These six issues are related to surface matching, weather location, surface normal, materials, loads, and schedules. The process in category three also identifies these issues as it uses IFC files and OpenStudio for translation. The extra step that is used in this approach does not add any extra complexities, as OpenStudio can translate to IFD for simulation in EnergyPlus. All new information is applied in OpenStudio before the second translation, so it is exported in the correct formatting. The complex model in Table 42 also identifies six issues. All these issues are identified by the low complexity model approach. Because the original approach for this process failed, solving these issues allows for another successful set of processes. The most common issues schedules and loads are re-implemented into the model through OpenStudio. This approach also contains the third most common issue, which is with the weather file not being applied. OpenStudio is the solution to this as well; it just needs to be manually applied. Materials do not export correctly when using the IFC schema, they are present but all set to default or close to default values. Material properties are replaced with actual values. Another problem, which appears only with IFC files is that some surfaces flip and are not recognised because they are now facing outwards. This issue only occurs with internally surface matched geometry. It can be solved by manually flipping them in a BEM editing software, like SketchUp.

\subsection{Effectiveness of Approaches}

All but one of the adjusted approaches manages to simulate successfully without any major errors. Although, this does not mean that all but one is effective. The only process that has a partial Middleware translation and simulation is exhibited in Table 41.

A few issues are identified from testing the three processes described in Noack et al. (2016). Mainly one of the most important pieces of information is that schedules and loads are not applied 
in OpenStudio through the gbXML export from Revit. Additionally, the problem with duplicate information causing geometry not to generate is seen. The second workflow solved this problem with a step ensuring correct information is applied and that incorrect information is removed as to stop it propagating. Overall, the approaches work well to translate information with only a couple of issues. This is one of the simpler processes being tested. The processes exhibited in Figure 41, Figure 44, and Figure 51 includes a step that promotes interoperable processes. This step takes the simulation results, applies them to the D4E repository, which is then used to generate the Revit model. In Noack et al. (2016) this process is not described nor is how the results actually change the input for future models. If this step could have been implemented a circular process could have been achieved.

The fourth approach identifies many problems with the transfer of information using an IFC file. The simple and complex models both identify similar issues, which for the most part only occur due to the file schema. Very little of this approach is adjusted from the original process; a lot of the actual translation also occurs within the BIMserver software, meaning changes cannot easily be made. Because the IFC schema is more complicated and does not contain certain material property information a large amount of manual manipulation was performed, making the approach not user-friendly.

The benefits of using a process that is considered an IDMM are shown through the testing of processes by Santos et al. (2017). With the low complexity model there were no sever errors causing incorrect results, however, there a small amount of manual adjustment is required to allow it to run. The benefits including being able to use the gbXML file directly, edit the geometry properties, and apply new schedules or loads automatically once set up, make this process unique. The VPL of Grasshopper allows multiple pre-set variables to be implemented and tested and even parametric simulation to be set up, although these are not tested in this workflow. The downside of this approach is seen with the complex model, if errors occur, there is no immediate way to know unless something looks incorrect in the visualization of the model results, or the error output file is opened directly and read. Due to the nature of the Middleware automatically outsourcing simulation to either OpenStudio or EnergyPlus, and then drawing those results back in, analysis is simpler for inexperienced modellers. This is because of the vast array of tools that can be applied to visualise the results like, an energy balance, results mapping on surfaces or zones, and ECM benefit calculators.

BIM promises a two-way, interoperable transition of information. From the process tested, this is not seen to be happening for BPS. Some outputs from the design tools are translatable only one 
Chapter Six: Discussion

way. For example, due to IFC only containing a whole wall R-value, changing one material property is no longer possible without additional calculations being run. 


\section{Chapter Six: Discussion}




\section{Chapter Seven: Conclusion}

The thesis aim was inspired by the many different attempts that have been made to translate Architectural models into Building Energy Models (BEM).

\subsection{Whether any Translation Processes Work?}

Within the aim of exploring the translating processes one of the objectives was to document whether any of them currently work. Nineteen processes for translating information from an Architectural model to a Building Energy Model were identified. Of these, seven had enough documentation that they could be tested, but one could not be made operational. For the remaining six processes at both the low and high levels of model complexity, following the documentation only two processes successfully navigate the Middleware stage to contain no errors. The Simulation software did not work with either of these two files. Manual intervention at the Middleware stage was required to progress to energy Simulation software files that ran successfully. This manual intervention consisted of two types of activity: editing the Middleware file itself, and editing options such as operational data in the Middleware.

The New Zealand BIMAccelerationCommittee (2019), like many equivalent international promoters of BIM, identifies energy analysis as a beneficial part of BIM. However, this study has shown that currently Building Information Models (BIM) cannot be used to perform Building (energy) Performance Simulation (BPS) analysis without major errors. With certain constraints, some Architectural modelling programs allow energy use to be calculated within very particular constraints. Design option modelling in the BPS is not facilitated. This suggests that future advancements in Architectural modelling and performance simulation should focus as a priority on this interoperability.

Full interoperability, where simulated models translate back to an Architectural design software were expected to play a bigger part in processes found. Four out of the 19 references seemed to have some interoperable process from viewing them. This is a process being pushed by the BIM Acceleration Committee in New Zealand as being possible. However, none of the tested processes could bring simulated information back to the original Architectural modelling program.

Processes that use the Industry Foundation Class (IFC) schema to translate information to a Middleware software produce more issues than those using Green Building standard XML (gbXML) schemas presented through Table 43. This may be because of how these files are constructed during export. IFC files are written from the top-down, while gbXML bottom-up. In IFC, geometry is generated first which materials, material layer, material layer set and properties sets are applied 
to in respective order. This means geometry is generated, the names of materials in the geometry are applied, widths of those materials are applied, a construction is generated from this information and named, and then properties are applied to the construction. The problem with this is that thermal properties are defined over a whole construction as one value. So, if one material is changed the whole construction need to be re-exported. The file sizes of IFC are much larger as everything is defined individually. In gbXML, the lowest level of detail is defined first. Each material has property values specific for BPS applied. Material names are then ordered to form layers that represent constructions with the addition of a few more properties values. Spaces are then defined through areas and volumes. Finally, each surface of geometry has a set of properties defined including name, surface type, construction ID reference, exposure to sun, and adjacent space ID.

There was a clear divide seen between the two types of building translation schema evaluated in this thesis. The dedicated approach of gbXML and generic approach of IFC identified similar issues; however, IFC contained a lot more of these problems because it communicates with all modelling programs at a lower quality. As the generic approaches contain more issues that take longer to solve, it is currently more complicated to generate an energy model out of IFC data. While the gbXML schema can only provide benefits for BPS related translations, it is the most viable way to provide the service.

\subsection{Whether there are any Common Problems or More Successful Approaches for Future Improvements}

Within the aim of exploring the translating processes the second objective was to document any common problems or more successful approaches. Eight different issues were identified from the 12 simulation tests (six processes, and two levels of model complexity). These eight issues are; loads, schedules, weather and location, materials, surface matching, surface normal, airflow, and spaces.

- Missing loads effect the buildings ability to calculate heat from people, lights, and equipment.

- Missing schedules effect when equipment is on, what temperature HVAC is heating or cooling to, or when occupants are present.

- Missing weather and location information means the building would not be placed in the desired climate, effecting temperature, lighting, and energy. 
- Missing material information and building constructions affect the thermal calculations on heat gain and loss through the envelope.

- Missing surface matching means that internal elements, which have different spaces on each side, would not be connected to each other, instead, it would be considered external.

- Incorrect surface normal effect the direction walls, floors, or roofs are orientated. Due to there being an internal and an external side of each element, external must always face outwards.

- Incorrect airflow means airflow rate being brought in through windows, mechanically, or through small cracks are set inconsistently.

- Duplicate spaces, which are considered the volume of a room, can be generated within a building. Both occurrences are automatically removed that results in no space being represented.

A detailed description of each was shown in Chapter 5. Not all processes suffered from each issue, but loads and schedules were a problem in every translation process. Some of the fewer identified issues are related to Airflow and Space ID.

Table 43: Simplified View of Identified Issues

\begin{tabular}{|c|c|c|c|c|c|c|c|c|c|c|c|}
\hline $\begin{array}{l}7 \\
0 \\
0 \\
0 \\
0 \\
0 \\
0\end{array}$ & 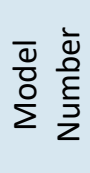 & 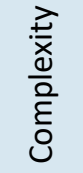 & $\begin{array}{l}n \\
0 \\
0 \\
0\end{array}$ & $\begin{array}{l}\frac{\tilde{d}}{5} \\
\frac{0}{0} \\
\frac{\tilde{d}}{U} \\
\text { v }\end{array}$ & $\begin{array}{l}\frac{1}{d} \\
\frac{1}{1} \\
\frac{1}{3} \\
3\end{array}$ & 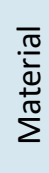 & 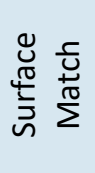 & 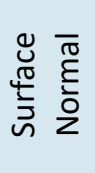 & $\frac{3}{\frac{0}{\frac{L}{4}}}$ & 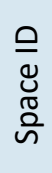 & $\begin{array}{l}\frac{5}{3} \\
\frac{c}{\frac{c}{y}} \\
\frac{5}{5}\end{array}$ \\
\hline \multirow{6}{*}{1} & \multirow{2}{*}{1} & Low & & & & & & & & & \\
\hline & & High & & & & & & & & & \\
\hline & \multirow{2}{*}{2} & Low & & & & & & & & & \\
\hline & & High & & & & & & & & & \\
\hline & \multirow{2}{*}{3} & Low & & & & & & & & & \\
\hline & & High & & & & & & & & & \\
\hline \multirow{2}{*}{2} & \multirow{2}{*}{4} & Low & & & & & & & & & \\
\hline & & High & & & & & & & & & \\
\hline \multirow{2}{*}{3} & \multirow{2}{*}{5} & Low & & & & & & & & & \\
\hline & & High & & & & & & & & & \\
\hline \multirow{4}{*}{4} & \multirow{2}{*}{6} & Low & & & & & & & & & \\
\hline & & High & & & & & & & & & \\
\hline & \multirow{2}{*}{7} & Low & & & & & & & & & \\
\hline & & High & & & & & & & & & \\
\hline
\end{tabular}

Seven out of the eight issues were found in both model complexities, leaving one unique issue to high complexity. The issue associated only with complex models is to do with Space IDs and occurs 
there due to internal floors not being used in the low complexity model. Interestingly it not only occurred in high complexity models but ones only using gbXML as the translation schema. Because both complexities contain similar issues, a more complex model should not produce any more issues.

There was only one issues relating to a specific model complexity. The issue with Airflow is identified only with processes using software and plugins for Grasshopper and Honeybee due to the way it applies ideal loads, infiltration, and ventilation rates. Surface normal issues are only introduced when an IFC file schema is used during the process.

In future research and development, these eight issues must specifically be tested. Avoiding problematic issues ensures Architect's models can be used in the translation process to conduct quantitative sustainable design.

All eight of the issues identified have a been successfully simulated due to manual solutions and some have shown the potential for automated ones. The solutions have been described in Chapter 6.

Simpler issues such as missing Schedules or Weather only require a small adjustment to the translation process. Manually solving this is not complicated, code could simply be written to automate the process of adding in text-based information. However, issues that are more complicated also arose. Surface matching was not always automatically completed. No processes based on the IFC file schema surface matched elements with either the low or high complexity model. The significance of this is that while some issues can be solved during a translation process with the addition of information, some require the user to spend a lot of time, manually fixing errors.

Not only does the increase in complexity of the models mean there are more surfaces and more zones, but also the amount of time to solve some issues dramatically increases. It is unfeasible to expect a busy Architect to use their time solving issues. This would put them off the idea of simulation; therefore, it would not be able to help them in design.

Table 44 presents all the processes tested, the result, and then the results if they are edited and a solution was implemented.

Table 44: Simplified Points of Failure and Success

\begin{tabular}{|c|c|c|c|c|c|c|c|c|c|c|c|c|c|c|c|c|}
\hline Category & \multicolumn{4}{|c|}{1} & \multicolumn{4}{|c|}{2} & \multicolumn{4}{|c|}{3} & \multicolumn{4}{|c|}{4} \\
\hline Complexity & \multicolumn{2}{|c|}{$\mathrm{L}$} & \multicolumn{2}{|c|}{$\mathrm{H}$} & \multicolumn{2}{|c|}{$L$} & \multicolumn{2}{|c|}{$\mathrm{H}$} & \multicolumn{2}{|c|}{$\mathrm{L}$} & \multicolumn{2}{|c|}{$\mathrm{H}$} & \multicolumn{2}{|c|}{$\mathrm{L}$} & \multicolumn{2}{|c|}{$\mathrm{H}$} \\
\hline Edited & $\mathrm{N}$ & $Y$ & $\mathrm{~N}$ & $Y$ & $\mathrm{~N}$ & $Y$ & $\mathrm{~N}$ & $Y$ & $\mathrm{~N}$ & $Y$ & $\mathrm{~N}$ & $Y$ & $\mathrm{~N}$ & $Y$ & $N$ & $Y$ \\
\hline
\end{tabular}


The low complexity model exported information correctly all the time, using all seven processes within the four approaches above. This, however, does not imply that all the required information was present. While the Simulation software ran after these automated translations and manual edits, much of the manual editing was to ensure the information about building materials, climate, operation, and so on was related to the original Architect's model. The complex model suffered the same lack of information transfer, but one process in category one process did not export correctly, even after manual editing in the Middleware.

The detail required in the Architect's model that is necessary for a Building Energy Simulation is geometry, adjacencies, constructions, and usage. The building geometry must contain the size, orientation, and element type e.g. roof, wall, floor. In addition, information is required about any attached geometry such as windows or doors. The adjacencies and boundaries of rooms must be defined so volumes and internal elements can be generated. Constructions and material properties are required for all elements so that BPS programs can calculate factors such as temperature, light level, or energy consumption. Lastly, to form a complete picture of energy performance the BPS program needs information on the usage data of the spaces, such as the number of light fittings, number of people, or period of occupancy. The Architectural models evaluated did not contain this extra information about the design, and yet it is implicit in much of the modelling that an Architect would engage in.

Consequently, when each process was manually manipulated to solve for identified issues and errors, successful simulations were generated. There seems no likely model complexity where this would not be possible. The only major barrier to translation of very complex Architecture Models to BEMs is the likely complexity and time cost of the manual edits. One process, from Noack et al. (2016) using IFC clearly does not produce a working Energy Model. Future research and development needs to focus on eliminating the requirement for manual editing:

1) of files format transfer so that the Simulation software input file will function;

2) of the file content to ensure that the Simulation software accurately reflects the location, construction, and operation of the proposed building. 
Chapter Seven: Conclusion 


\section{References}

ALSAADANI, S. \& BLEIL DE SOUZA, C. 2016. Of collaboration or condemnation? Exploring the promise and pitfalls of architect-consultant collaborations for building performance simulation. Energy Research \& Social Science, 19, 21-36.

ALSAADANI, S. \& BLEIL DE SOUZA, C. 2018. Architect-BPS consultant collaborations: Harmony or hardship? Journal of Building Performance Simulation, 11, 391-413.

ATTIA, S., BELTRÁN, L., DE HERDE, A. \& HENSEN, J. "Architect friendly": A comparison of ten different building performance simulation tools. 11th International IBPSA Conference Building Simulation 2009, BS 2009, 2009 Glasgow. 204-211.

ATTIA, S., HENSEN, J. L. M., BELTRÁN, L. \& DE HERDE, A. 2012. Selection criteria for building performance simulation tools: contrasting architects' and engineers' needs. Journal of Building Performance Simulation, 5, 155-169.

BAZJANAC, V. 2008. IFC BIM-Based Methodology for SemiAutomated Building Energy Performance Simulation. International Conference on Information Technology in Construction. Santiago, Chile.

BIMACCELERATIONCOMMITTEE 2019. A Guide To Enabling Bim On Built Assets 2019 Third Edition. The New Zealand BIM Handbook. BIMinNZ.

BIMSERVER. 2014. Open source BIMserver. Available: http://bimserver.org/about/ [Accessed 2019].

BOLAND, A., CHERRY, G. \& DICKSON, R. 2014. Doing a Systematic Review A Student's Guide, SAGE.

BRAASCH, E. 2016. The feasibility of 'building performance sketching' within the building design process. Master of Building Science, Victoria University of Wellington.

BRAASCH, E. \& DONN, M. 2016. The feasibility of implementing 'building performance sketching' within the building design process through the use of the distributed model method. Architectural Science Association. The University of Adelaide.

BUILDINGSMART. 2019. IFC View Definitions [Online]. Website: BuildingSmart. Available: http://www.buildingsmart-tech.org/specifications/ifc-view-definition [Accessed $1 / 5 / 2019]$.

CEREZO, C., DOGAN, T. \& REINHART, C. Towards standarized building properties template files for early design energy model generation. 2014 ASHRAE/IBPSA-USA Building Simulation Conference, 2014. American Society of Heating, Refrigeration, and Air-Conditioning Engineers (ASHRAE), 25-32.

CHAISUPARASMIKUL, P. Bidirectional interoperability between CAD and energy performance simulation through virtual model system framework. Synthetic Landscapes - ACADIA 2006 International Conference, 2006 Louisville, KY. 232-250.

DIMITRIOU, V., FIRTH, S. K., HASSAN, T. M. \& FOUCHAL, F. 2016. BIM enabled building energy modelling: development and verification of a GBXML to IDF conversion method. IBPSAEngland. Newcastle: IBPSA.

DONG, B., LAM, K. P., HUANG, Y. C. \& DOBBS, G. M. A comparative study of the IFC and gbXML informational infrastructures for data exchange in computational design support environments. Building Simulation, 2007.

GANDA, S. 2019. A Life Cycle Assessment of Medium Density Houses in New Zealand. Masters of Building Science, Victoria University of Wellington.

GIANNAKIS, G., LILIS, G., KONTES, G., GARCÍA-FUENTES, M., VALMASEDA, C. \& ROVAS, D. 2015. A methodology to automatically generate geometry inputs for Energy Performance Simulation from IFC BIM models.

HEEP 2010. Energy use in New Zealand households - final HEEP report. In: ISAACS, N., CAMILLERI, M., BURROUGH, L., POLLARD, A., SAVILLE-SMITH, K., FRASER, R., ROSSOUW, P. \& JOWETT, J. (eds.) Energy use in New Zealand households. BRANZ. 
ICGL. Transparency in Grey Literature. International Conference on Grey Literature, December 672010 National Technical Library, Prague, Czech Republic.

JALAEI, F. \& JRADE, A. J. I. 2014. Integrating Building Information Modeling (BIM) and Energy Analysis Tools with Green Building Certification System to Conceptually Design Sustainable Buildings. 19, 494-519.

JEONG, W., KIM, J. B., CLAYTON, M. J., HABERL, J. S. \& YAN, W. 2016. A framework to integrate object-oriented physical modelling with building information modelling for building thermal simulation. Journal of Building Performance Simulation, 9, 50-69.

JONES, N., MCCRONE, C., WALTER, B., PRATT, K. \& GREENBERG, D. Automated Translation And Thermal Zoning Of Digital Building Models For Energy Analysis. International Building Performance Simulation Association, 2013 Chambéry, France.

KAMEL, E. \& MEMARI, A. M. 2019. Review of BIM's application in energy simulation: Tools, issues, and solutions. Automation in Construction, 97, 164-180.

KIM, J. B., JEONG, W., CLAYTON, M. J., HABERL, J. S. \& YAN, W. 2015. Developing a physical BIM library for building thermal energy simulation. Automation in Construction, 50, 16-28.

KOTA, S., HABERL, J. S., CLAYTON, M. J. \& YAN, W. 2014. Building Information Modeling (BIM)based daylighting simulation and analysis. Energy and Buildings, 81, 391-403.

KUMAR, S. \& MAHDAVI, A. 2001. Integrating thermal comfort field data analysis in a case-based building simulation environment. Building and Environment, 36, 711-720.

LBNL 2018. EnergyPlus Input Output Reference. 40101eaafd ed. U.S. Department of Energy: Lawrence Berkeley National Laboratory.

LILIS, G. N., GIANNAKIS, G. I. \& ROVAS, D. V. 2017. Automatic generation of second-level space boundary topology from IFC geometry inputs. Automation in Construction, 76, 108-124.

MAILE, T., O'DONNELL, J., BAZJANAC, V. \& ROSE, C. BIM - Geometry modelling guidelines for building energy performance simulation. 13th Conference of the International Building Performance Simulation Association, BS 2013, 2013 Chambery. 3242-3249.

NBIMS 2015. National BIM Standard - US. National Institute of Building Sciences.

NEGENDAHL, K. 2015. Building performance simulation in the early design stage: An introduction to integrated dynamic models. Automation in Construction, 54, 39-53.

NOACK, F., KATRANUSCHKOV, P., SCHERER, R., DIMITRIOU, V., FIRTH, S. K., HASSAN, T. M., RAMOS, N., PEREIRA, P., MALO, P. \& FERNANDO, T. Technical challenges and approaches to transfer building information models to building energy. In: CHRISTODOULOU, S. E. \& SCHERER, R., eds. 11th European Conference on Product and Process Modelling, ECPPM 2016, 2016. CRC Press/Balkema, 355-362.

NZS4214 2006. Methods of Determining the Total Thermal Resistance of Parts of Buildings. Standards New Zealand.

NZS4218 2009. Thermal Insulation - Housing and Small Buildings. Standards New Zealand.

PRATT, K. B., JONES, N. L., SCHUMANN, L., BOSWORTH, D. E. \& HEUMANN, A. D. Automated translation of architectural models for energy simulation. Symposium on Simulation for Architecture and Urban Design 2012, SimAUD 2012, Part of the 2012 Spring Simulation Multiconference, SpringSim 2012, 2012 Orlando, FL. 1-8.

RAMAJI, I. J., MESSNER, J. I. \& LEICHT, R. M. 2016. Leveraging Building Information Models In IFC To Perform Energy Analysis In Openstudio. Building Performance Modeling Conference. Salt Lake City: IBPSA-USA.

ROUDSARI, M. \& MACKEY, C. 2019. Ladybug Tools [Online]. Online. Available: https://www.ladybug.tools/ [Accessed].

SANTOS, L., SCHLEICHER, S. \& CALDAS, L. 2017. Automation of CAD models to BEM models for performance based goal-oriented design methods. Building and Environment, 112, 144158.

SOEBARTO, V., HOPFE, C. J., CRAWLEY, D. \& RAWAL, R. Capturing the views of architects about building performance simulation to be used during design processes. 14th Conference of 
International Building Performance Simulation Association, BS 2015, 2015. International Building Performance Simulation Association, 1480-1487.

STRUCK, C., DE WILDE, P. J. C. J., HOPFE, C. J. \& HENSEN, J. L. M. 2009. An investigation of the option space in conceptual building design for advanced building simulation. Advanced Engineering Informatics, 23, 386-395.

TOTH, B., SALIM, F., BURRY, J., FRAZER, J., DROGEMULLER, R. \& BURRY, M. 2011. Energy-Oriented Design Tools for Collaboration in the Cloud. International Journal of Architectural Computing, 9, 339-359.

WANG, D. \& GROAT, L. N. 2013. Architectural Research Methods, Somerset, UNITED STATES, John Wiley \& Sons, Incorporated.

WEISE, M., LIEBICH, T., SEE, R., BAZJANAC, V., LAINE, T. \& WELLE, B. 2011. Implementation Guide: Space Boundaries For Energy Analysis. http://www.blisproject.org/IAIMVD/documents/Space Boundaries for Energy Analysis v1.pdf.

WELLE, B., HAYMAKER, J. \& ROGERS, Z. 2011. ThermalOpt: A methodology for automated BIMbased multidisciplinary thermal simulation for use in optimization environments. Building Simulation, 4.

WEYTJENS, L. \& VERBEECK, G. Towards 'architect-friendly' energy evaluation tools. 2010 Spring Simulation Multiconference, SpringSim'10, 2010 Orlando, FL.

XIE, X. \& GOU, Z. 2017. Building performance simulation as an early intervention or late verification in architectural design: Same performance outcome but different design solutions. Journal of Green Building, 12, 45-61.

YEANG, K. \& LAM, K. P. 2009. Computational building performance modelling and ecodesign. Architectural Design, 79, 126-129. 


\section{Appendix One}

Table 46: Issues and Assessment from Identified References

\begin{tabular}{|c|c|c|c|c|c|c|c|c|}
\hline \multirow[b]{2}{*}{ Reference } & \multicolumn{8}{|c|}{ Issues } \\
\hline & 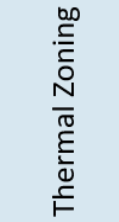 & 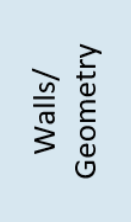 & $\begin{array}{l}\frac{n}{\frac{N}{2}} \\
\frac{0}{2} \\
\frac{\pi}{2} \\
\sum\end{array}$ & 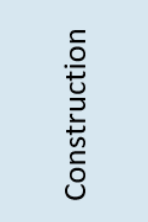 & 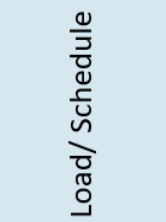 & 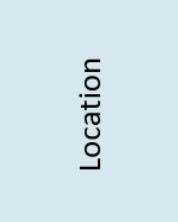 & $\begin{array}{l}\stackrel{\mathbb{Z}}{\pi} \\
0 \\
\stackrel{0}{ \pm} \\
\stackrel{0}{0} \\
\frac{0}{0} \\
0\end{array}$ & $\begin{array}{l}\underset{\pi}{\pi} \\
0 \\
0 \\
. \frac{1}{n} \\
\stackrel{\mathscr{n}}{\Sigma}\end{array}$ \\
\hline (Kota et al., 2014) & No & $\begin{array}{l}\text { Yes, 2D, } \\
\text { Revit }\end{array}$ & Yes, Revit & No & No & $\begin{array}{c}\text { Yes, } \\
\text { Climate File }\end{array}$ & No & No \\
\hline $\begin{array}{c}\text { (Chaisuparasmiku } \\
\text { I, 2006) }\end{array}$ & $\begin{array}{l}\text { Yes, } \\
\text { Revit }\end{array}$ & $\begin{array}{c}\text { Yes, 2D, } \\
\text { Revit }\end{array}$ & Yes, Revit & Yes, Revit & $\begin{array}{c}\text { Yes, } \\
\text { DOE-2 }\end{array}$ & $\begin{array}{c}\text { Yes, } \\
\text { Climate File }\end{array}$ & No & No \\
\hline $\begin{array}{c}\text { (Cerezo et al., } \\
\text { 2014) }\end{array}$ & $\mathrm{n} / \mathrm{a}$ & $\mathrm{n} / \mathrm{a}$ & Yes, No & Yes, No & Yes, No & $\mathrm{n} / \mathrm{a}$ & $\mathrm{n} / \mathrm{a}$ & $\mathrm{n} / \mathrm{a}$ \\
\hline $\begin{array}{l}\text { (Yeang and Lam, } \\
\text { 2009) }\end{array}$ & No & No & No & No & No & No & No & No \\
\hline $\begin{array}{c}\text { (Kamel and } \\
\text { Memari, 2019) }\end{array}$ & $\begin{array}{l}\text { Yes, } \\
\text { Revit }\end{array}$ & $\begin{array}{c}\text { Yes, 2D, } \\
\text { Revit }\end{array}$ & Yes, Revit & Yes, Revit & $\begin{array}{l}\text { Yes, } \\
\text { Revit }\end{array}$ & Yes, Revit & Yes & Yes \\
\hline $\begin{array}{c}\text { (Santos et al., } \\
\text { 2017) }\end{array}$ & No & $\begin{array}{l}\text { Yes, 2D, } \\
\text { Rhino }\end{array}$ & Yes, HB & No & Yes, HB & No & No & No \\
\hline $\begin{array}{c}\text { (Kumar and } \\
\text { Mahdavi, 2001) }\end{array}$ & No & No & No & No & No & $\begin{array}{l}\text { Yes, Climate } \\
\text { File }\end{array}$ & No & No \\
\hline $\begin{array}{l}\text { (Maile et al., } \\
\text { 2013) }\end{array}$ & Yes, No & $\begin{array}{c}\text { Yes, 3D, } \\
\text { CAD }\end{array}$ & No & Yes, No & No & No & No & No \\
\hline $\begin{array}{c}\text { (Noack et al., } \\
2016)\end{array}$ & $\begin{array}{l}\text { Yes, } \\
\text { Revit }\end{array}$ & $\begin{array}{l}\text { Yes, No, } \\
\text { Revit }\end{array}$ & No & Yes, Revit & $\begin{array}{c}\text { Yes, } \\
\text { EnergyPlus }\end{array}$ & $\begin{array}{c}\text { Yes, } \\
\text { EnergyPlus }\end{array}$ & No & Yes \\
\hline (Pratt et al., 2012) & Yes, No & $\begin{array}{l}\text { Yes, 2D, } \\
\text { No }\end{array}$ & No & Yes, No & No & $\begin{array}{c}\text { Yes, } \\
\text { Climate File }\end{array}$ & Yes & Yes \\
\hline (Bazjanac, 2008) & Yes, No & $\begin{array}{l}\text { Yes, 2D, } \\
\text { No }\end{array}$ & Yes, No & Yes, No & Yes, No & $\begin{array}{c}\text { Yes, } \\
\text { Climate File }\end{array}$ & No & Yes \\
\hline $\begin{array}{c}\text { (Giannakis et al., } \\
\text { 2015) }\end{array}$ & $\begin{array}{l}\text { Yes, } \\
\text { Revit }\end{array}$ & $\begin{array}{c}\text { Yes, 3D, } \\
\text { Revit }\end{array}$ & Yes, Revit & Yes, Revit & No & No & No & No \\
\hline $\begin{array}{c}\text { (Jeong et al., } \\
\text { 2016) }\end{array}$ & $\begin{array}{l}\text { Yes, } \\
\text { Revit }\end{array}$ & $\begin{array}{c}\text { Yes, 2D, } \\
\text { Revit }\end{array}$ & Yes, Revit & Yes, Revit & $\begin{array}{c}\text { Yes, } \\
\text { Modelica }\end{array}$ & $\begin{array}{l}\text { Yes, } \\
\text { Revit }\end{array}$ & No & Yes \\
\hline $\begin{array}{c}\text { (Jones et al., } \\
\text { 2013) }\end{array}$ & No & $\begin{array}{c}\text { Yes, 3D, } \\
\text { No }\end{array}$ & Yes, No & No & Yes, No & No & Yes & Yes \\
\hline (Kim et al., 2015) & $\begin{array}{l}\text { Yes, } \\
\text { Revit }\end{array}$ & $\begin{array}{c}\text { Yes, 3D, } \\
\text { Revit }\end{array}$ & Yes, Revit & Yes, Revit & $\begin{array}{c}\text { Yes, } \\
\text { Modelica }\end{array}$ & $\begin{array}{l}\text { Yes, } \\
\text { Revit }\end{array}$ & No & Yes \\
\hline (Lilis et al., 2017) & $\begin{array}{l}\text { Yes, } \\
\text { Revit }\end{array}$ & $\begin{array}{c}\text { Yes, 3D, } \\
\text { Revit }\end{array}$ & Yes, Revit & Yes, No & No & No & No & Yes \\
\hline $\begin{array}{c}\text { (Welle et al., } \\
\text { 2011) }\end{array}$ & Yes, DP & $\begin{array}{l}\text { Yes, 2D, } \\
\text { DP }\end{array}$ & Yes, DP & Yes, DP & No & $\begin{array}{l}\text { Yes, } \\
\text { DP }\end{array}$ & No & Yes \\
\hline $\begin{array}{c}\text { (Ramaji et al., } \\
\text { 2016) }\end{array}$ & $\begin{array}{l}\text { Yes, } \\
\text { Revit }\end{array}$ & $\begin{array}{c}\text { Yes, 2D, } \\
\text { Revit }\end{array}$ & Yes Revit & Yes, Revit & No & No & No & No \\
\hline $\begin{array}{c}\text { (Dimitriou et al., } \\
\text { 2016) }\end{array}$ & $\begin{array}{l}\text { Yes, } \\
\text { Revit }\end{array}$ & $\begin{array}{c}\text { Yes, 2D, } \\
\text { Revit }\end{array}$ & Yes, Revit & Yes, Revit & No & $\begin{array}{l}\text { Yes, Climate } \\
\text { File }\end{array}$ & No & Yes \\
\hline
\end{tabular}




\begin{tabular}{|c|c|}
\hline Building Performance Simulation Tool: & An Architect should be able to: \\
\hline \multicolumn{2}{|l|}{ Data Input } \\
\hline Limited data input & Select defaults or input small amounts of data quickly \\
\hline Quick data input & “ \\
\hline Input in the language of the Architect & " \\
\hline Use of defaults to limit and facilitate data-entry & " \\
\hline Simple and intuitive input process & $\begin{array}{l}\text { Intuitively input or change data to create alternative } \\
\text { design options during the early design stage }\end{array}$ \\
\hline Easy data review/change & " \\
\hline Easy create alternative designs/options & " \\
\hline Extensive library/database of building components & $\mathrm{n} / \mathrm{a}$ \\
\hline Input consistent with early design phase & " \\
\hline From general to detail & $?$ \\
\hline Graphical representation of building geometry: & Model a 3-D representation of a building \\
\hline 3D modeller in simulation tool & $\mathrm{n} / \mathrm{a}$ \\
\hline Possibility to import 3D CAD files & $\mathrm{n} / \mathrm{a}$ \\
\hline Possibility to import from Sketch-Up & $\mathrm{n} / \mathrm{a}$ \\
\hline Input via drawing software & $\mathrm{n} / \mathrm{a}$ \\
\hline \multicolumn{2}{|l|}{ Output } \\
\hline Easy interpretation (language of Architects) & $\begin{array}{l}\text { Interoperate the results and conformance with building } \\
\text { codes via a graphical representation }\end{array}$ \\
\hline Graphical representation of output & " \\
\hline Conformance with building codes and regulations & " \\
\hline Impact of decisions/parameters (uncertainty/sensitivity) & $\begin{array}{l}\text { Understand and communicate the impact of design } \\
\text { decisions using output information }\end{array}$ \\
\hline Simple but supportive information for design decisions & “ \\
\hline Convincing output, to communicate with clients & “" \\
\hline Clearly indicate problem area(s) & $\mathrm{n} / \mathrm{a}$ \\
\hline Benchmarking & $\mathrm{n} / \mathrm{a}$ \\
\hline Output displayed in 3D building model & $\mathrm{n} / \mathrm{a}$ \\
\hline Generate reports for alternative designs/options & “ \\
\hline Reliability of the output & Understand the accuracy of output \\
\hline Adapted for different design phases & “ \\
\hline \multicolumn{2}{|l|}{ Interface } \\
\hline Visual communication of GUI & Use a simple intuitive GUI \\
\hline Clear, intuitive, and flexible navigation & " \\
\hline Clearly structured with a restrained set of functions & "“ \\
\hline \multicolumn{2}{|l|}{ Usability in Development Process (DP) } \\
\hline Minimally interrupt the DP & $\begin{array}{l}\text { Use a simplistic tool during the development process to } \\
\text { obtain results }\end{array}$ \\
\hline Data-input in tune with DP & "“ \\
\hline Simplicity & "“ \\
\hline Minimal time required to operate the tool & " \\
\hline Adapted for use in early design & " \\
\hline Quickly obtain solutions & “ \\
\hline Quickly and easily create, test and compare alternatives & " \\
\hline Real-time feedback on design decisions and changes & " \\
\hline Provide guidelines & " \\
\hline \multicolumn{2}{|l|}{ General } \\
\hline Adaptable default values & Learn the intuitive features of a new tool \\
\hline Highly visual & “ \\
\hline Transparency of the tool & “ \\
\hline Ease and intuitive in use & “ \\
\hline Short calculation time & " \\
\hline Easy to learn & " \\
\hline Adequate for local usage (units/materials/...) & Be able to apply correct materials \\
\hline Easy to use after long time of non-use & " \\
\hline
\end{tabular}




\section{Appendix One}

Table 48: Insulation Requirements for Wellington, New Zealand (NZS4218, 2009)

\begin{tabular}{|l|l|}
\hline Building Element & Climate Zone 2 \\
\hline Roof & $\mathrm{R}-2.9$ \\
\hline Wall & $\mathrm{R}-1.9$ \\
\hline Floor & $\mathrm{R}-1.3$ \\
\hline Windows and Glazing & $\mathrm{R}-0.26$ \\
\hline Skylights & $\mathrm{R}-0.26$ \\
\hline
\end{tabular}




\section{Appendix Two}

\section{Appendix Two}

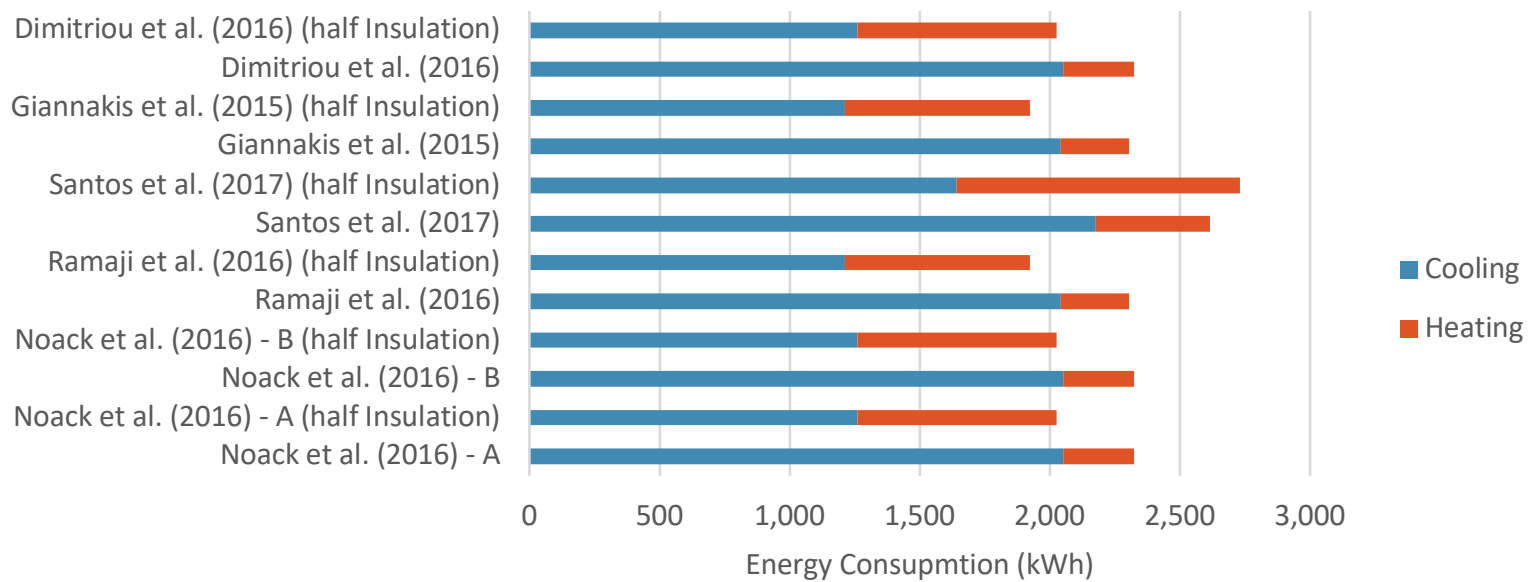

Figure 59: Low Complexity Model QA of Energy Consumption

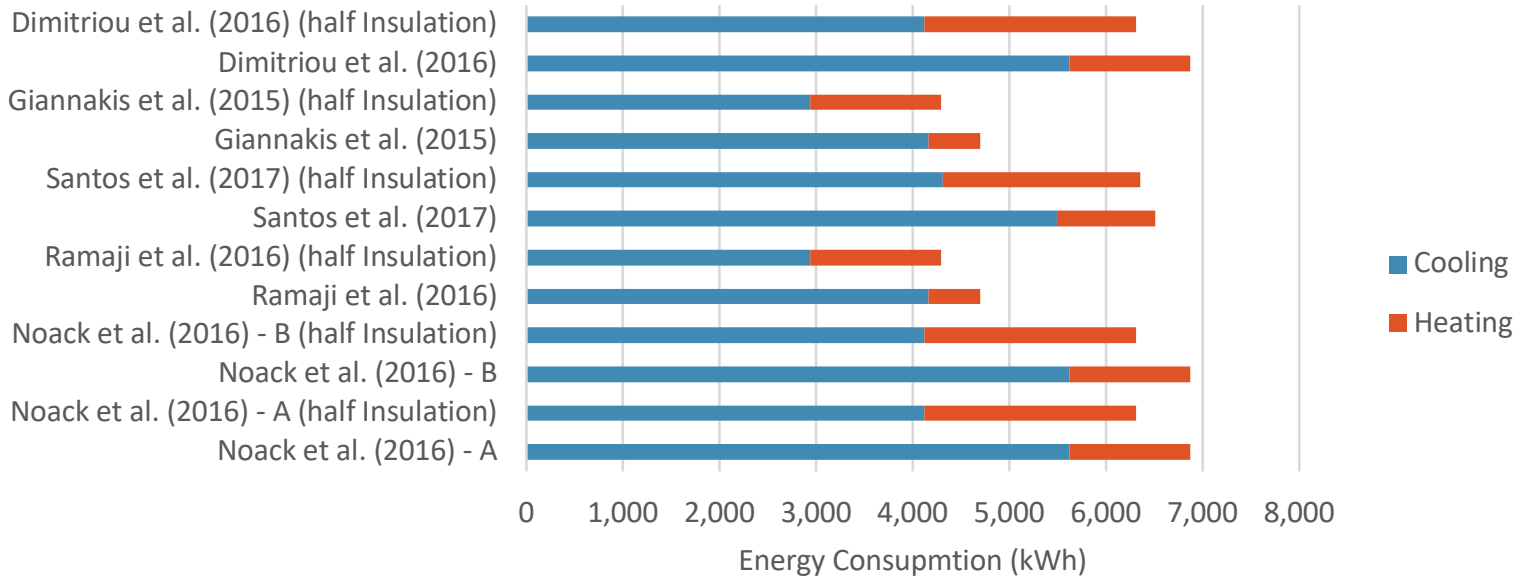

Figure 60: High Complexity Model QA of Energy Consumption

<Surface surfaceType="Ceiling" constructionIdRef="aim1290" id="aim1477">

<AdjacentSpaceld spaceldRef="aim0077" />

<AdjacentSpaceld spaceldRef="aim0077" />

<RectangularGeometry id="aim1478">

Figure 61: Original gbXML Ceiling Export 
Appendix Two

OpenStudioApp
Errors or warnings occurred on gbXML import.
\begin{tabular}{l}
\hline OK \\
Surface has two adjacent spaces which are the \\
same space 'aim0093', will not be translated. \\
Surface has two adjacent spaces which are the \\
same space 'aim0163', will not be translated. \\
Surface has two adjacent spaces which are the
\end{tabular}

Figure 62: OpenStudio Errors and Warning 


\section{Appendix Three}

Construction Set

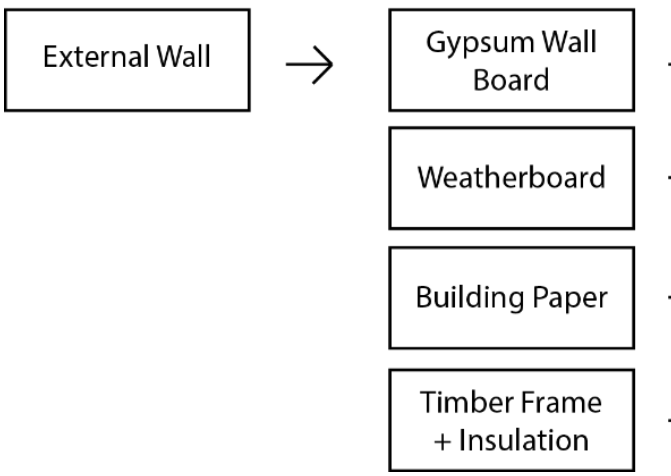

Properties

Thickness: $3 \mathrm{~m}$

$\rightarrow$ Conductivity: $0.001 \mathrm{~W} / \mathrm{mK}$

Density0.001 kg/m3

Thickness: $3 \mathrm{~m}$

$\rightarrow$ Conductivity: $0.001 \mathrm{~W} / \mathrm{mK}$

Density0.001 kg/m3

Thickness: $3 \mathrm{~m}$

$\rightarrow$ Conductivity: $0.001 \mathrm{~W} / \mathrm{mK}$

Density $0.001 \mathrm{~kg} / \mathrm{m} 3$

Thickness: $3 \mathrm{~m}$

$\rightarrow$ Conductivity: $0.001 \mathrm{~W} / \mathrm{mK}$

Density0.001 kg/m3

Figure 63: Incorrect Construction Details from IFC Import

Table 49: IFC vs Revit Components

\begin{tabular}{|l|c|}
\hline IFC Identifier & Revit Component \\
\hline IFC Slab & Internal or External Floor \\
\hline IFC Wall & Internal or External Wall \\
\hline IFC Door & Internal or External Door \\
\hline IFC Window & Internal or External Window \\
\hline IFC Roof & External Roof \\
\hline n/a & Internal Ceiling \\
\hline
\end{tabular}

Table 50: Boundary Surfaces Not Generated With IFC

\begin{tabular}{|c|c|c|}
\hline IFC Identifier & Boundary Condition & Boundary Surface \\
\hline IFC Slab & Internal & Not Generated \\
\hline IFC Wall & Internal & Not Generated \\
\hline IFC Door & Internal & Not Generated \\
\hline IFC Window & Internal & Not Generated \\
\hline
\end{tabular}

Table 51: Initial Energy Results from Ramaji et al. (2016)

\begin{tabular}{|c|c|c|c|c|}
\hline Floor Area $\left(\mathrm{m}^{2}\right)$ & Lighting $(\mathrm{kWh})$ & Equipment $(\mathrm{kWh})$ & Heating $(\mathrm{kWh})$ & Total $\left(\mathrm{kWh} / \mathrm{m}^{2}\right)$ \\
\hline $100,000,000$ & $1,277,550,694$ & $2,628,000,038$ & $73,476,944,444$ & 773 \\
\hline
\end{tabular}

